\title{
An extended finite element method (XFEM) for linear elastic fracture with smooth nodal stress
}

\author{
X. Peng ${ }^{1}$, S. Kulasegaram ${ }^{1}$, S. P. A. Bordas ${ }^{1,3}$, S. C. $\mathbf{W u}^{2}$ \\ Institute of Mechanics and Advanced materials, Cardiff University, CF24 3AA, UK \\ ${ }^{2}$ State Key Laboratory of Traction Power, Southwest Jiaotong University, Chengdu, 610031, \\ China \\ ${ }^{3}$ Université du Luxembourg, Faculté des Sciences, de la Technologie et de la Communication, \\ 6, rue Richard Coudenhove-Kalergi, L-1359 Luxembourg - Research Unit in Engineering \\ Science Campus Kirchberg, G 007
}

\begin{abstract}
In this paper, we present a method to achieve smooth nodal stresses in the XFEM. This method was developed by borrowing some ideas from the 'twice interpolating approximations' (TFEM) by Zheng et al (2011). The salient feature of the method is to introduce an 'average' gradient into the construction of the approximation, resulting in improved solution accuracy, both in the vicinity of the crack tip and in the far field. Due to the higher-order polynomial basis provided by the interpolants, the new approximation enhances the smoothness of the solution without requiring an increased number of degrees of freedom. This is particularly advantageous for low-order elements and in fracture mechanics. Since the new approach adopts the same mesh discretization, i.e. simplex meshes, it can be easily extended to various problems and is easily implemented. We also discuss the increased bandwidth which is a drawback of the present method. Numerical tests show that the new method is as robust as the XFEM, considering precision, model size and post-processing time. By comparing the results from the present method with the XFEM for crack propagation in homogeneous materials, we conclude that for two-dimensional problems, the proposed method tends to be an efficient alternative to the classical XFEM, bypassing any postprocessing step to obtain smooth modal stress fields and providing a direct means to compute local stress error measures.
\end{abstract}

Keywords: Double-interpolation approximation; higher-order element; smooth nodal stress; extended finite element method; crack propagation.

\section{Introduction}

The extended finite element method (XFEM)[1] is a versatile approach to model strong discontinuities and singularities that exist in linear elastic fracture mechanics. In the XFEM, the approximation of the displacement field is decomposed into a regular part and an additional part (enriched part). The enriched part carries specific information or the solution such as discontinuity or singularity, through additional degrees of freedom (DOFs) associated with enriched nodes. This provides great flexibility to model cracks since alignment of the mesh and cracks is unnecessary. The modeling procedure is simplified since the remeshing operations are no longer needed. The XFEM for fracture has been the topic of substantial developments over past decades in 3D [2][3][4], nonlinear problems [5][6] and dynamics problems [7], and has been utilized for assessing the damage tolerance of complex structures in industrial applications [8]. A posteriori error indicators were proposed by Bordas and Duflot [9][10][11][12]. C ++ libraries [13] as well as commercial packages [14][15][16] were developed for the XFEM. A close cousin

\footnotetext{
`Corresponding author. Tel:(+352)4666445567 Fax: $(+352) 46664435567$

E-mail address:stephane.bordas@alum.northwestern.edu
} 
of the XFEM called generalized finite element method (GFEM), was also proposed and applied for crack modeling (see for example [17]).

We start by reviewing some of the most salient and recent advance in enriched finite elements. Significant effort has been expended towards improving the accuracy and robustness of this method. In the standard XFEM, a local partition of unity is adopted, which means only certain nodes are enriched. This results in some elements (the blending elements) consisting of both regular and enriched nodes not fulfilling partition of unity. The existence of blending elements decreases accuracy and convergence rates. Chessa et al [18] developed an enhanced strain formulation which suppressed unwanted blending effects [19]. Gracie et al [20] proposed the discontinuous Galerkin method aimed at eliminating the source of error in blending elements. More such attempts can be read in [21][22].

In terms of integration, the additional non-polynomial enrichment functions in the approximation space make the quadrature of the stiffness matrix of enriched elements and blending elements more delicate. Singularities, sharp gradients in the crack tip enrichment functions add to the complexity of numerical integration. The traditional procedure to perform the integration is to sub-divide the enriched elements and blending elements into quadrature subcells[1]. Ventura [23] proposed an approach to eliminate the quadrature subcells via replacing nonpolynomial functions by 'equivalent' polynomials. But this method is only exact for triangular and tetrahedral elements. Another efficient integration scheme was proposed by transforming the domain integration into contour integration in [22]. Natarajan et al [24] developed a new numerical integration for arbitrary polygonal domains in 2D. In this method, each part of the elements that are cut or intersected by a discontinuity is conformally mapped onto a unit disc using the Schwarz-Christoffel mapping. In the smoothed XFEM, interior integration is transformed into boundary integration, and sub-dividing becomes unnecessary [25]. Laborde et al [26] adopted the almost polar integration within crack tip enriched elements, which improves the convergence rate.

Another issue observed in the original version of XFEM is the non-optimal convergence rate. One improvement is to use geometrical enrichment [26], i.e., the enrichment of a set of nodes within a radial domain around the crack tip, and the whole dimension is independent of the mesh size. Nevertheless, this approach deteriorates the condition number of the stiffness matrix, which somewhat limits its application to 3D problems. In order to reduce the condition number, effective preconditioners were proposed by Béchet [27] and Menk et al [28].

Apart from XFEM which broadly aims at providing approximations which are tailored to the solution, based on a priori knowledge about the solution, a number of interpolation methods have been developed in order to improve the efficiency of standard non-enriched FE methods. An example is the need for $C^{1}$ continuous approximations, for instance, to solve problems where continuity of the first derivative of the unknown field is required. This is the case for higherorder gradient models, such as gradient elasticity [29], Kirchhoff-love shell models [30]. Methods satisfying this need include mesh-based and mesh-free methods [31]. In terms of higher-order continuous FEM, Papanicolopulos and Zervos [32][33] created a series of triangular elements with $C^{1}$ continuous interpolation properties. Fischer et al [34] compared the performance of $C^{1}$ finite elements and the $C^{1}$ natural element method (NEM) applied to non-linear gradient elasticity. Various meshfree methods were introduced and used widely in engineering problems. The element free Galerkin method (EFG) [35] adopts the moving least-squares approximations to construct trial and test functions which can easily obtain higher continuous approximations. One similar method is the reproducing kernel particle method (RKPM)[36]. The meshfree radial basis functions method (RBFs) [37] utilizes radial basis functions to interpolate scattered nodal data and was employed with the point interpolation method (PIM) by Liu et al. The radial PIM (RPIM)[38][39][40], consists of both a radial basis and a polynomial basis in the approx- 
imation, which can avoid the singularity of the moment matrix arising for polynomial bases. The maximum-entropy method (MAXENT) proposed by Arroyo and Ortiz are a relatively new approximation functions based on maximizing Shannon entropy of the basis funcions[41][42] and has been incorporated with extrinsic enrichment to study the convergence for linear elastic fracture [43]. Liu et al developed a smoothed FEM (SFEM). The SFEMs can be classified as node-based, edge-based and face-based smoothed FEM. Researchers subsequently investigated the new methods to model discontinuities using partition of unity enrichment: extended SFEM [25][44][45][46][47][[48].

The goal of this work has been to construct simple approximations able to

- provide $C^{1}$ continuity almost everywhere;

- provide Kronecker delta property;

- rely on simplex meshes which are easily generated;

- be cheap to construct and integrate numerically;

- enable the treatment of propagating cracks with minimal remeshing.

This approximation procedure shares the attractive features of XFEM and higher-order continuous approximations. Two consecutive stages of interpolation are used in the construction of this approximation. The first stage of interpolation is performed by Lagrange interpolation to obtain nodal variables and nodal gradients. The problem field is reproduced in the latter interpolation using the nodal values and gradients derived from the previous interpolation. The re-constructed trial functions will maintain $C^{1}$ continuity at the nodes [49]. Cubic polynomials are contained in the space without increasing the total number of DOFs. This feature enhances the ability of the method to reproduce the solution near the crack tip [50] and improves the accuracy per DOF, The price to pay is increased computational expense per DOF, as discussed later in the paper. Analogous to meshfree methods, nodal stresses can be calculated in a straightforward manner without any post-processing.

The paper is organised as follows. In section 2 , the unenriched formulation for $1 \mathrm{D}$ and $2 \mathrm{D}$ is systematically introduced with a 1D bar example. Section 3 presents the discretized formulation of the enriched version of the proposed approximation for linear elastic fracture mechanics. Several numerical examples are presented to illustrate the advantages and limitations of the double-interpolation FEM (DFEM) and XFEM (XDFEM) in section 4. Finally, in section 5, concluding remarks are made with pointers to possible future work.

\section{The double-interpolation approximation}

\subsection{D approximation by double-interpolation}

The basic idea of the double-interpolation approximation is to interpolate the unknown fields, using both the primary nodal values and nodal gradients, which are generated by the finite element interpolation in simplex mesh discretization. The proposed 1D double-interpolation is comparable to Hermite interpolants. Figure 1 shows a 1D domain which is discretized by six $1 \mathrm{D}$ elements. For the point of interest $x$ in element $e_{3}$, the numerical value of the displacement can be interpolated by

$$
\forall x \in[0, \ell], \quad u^{h}(x)=\phi_{I}(x) u^{I}+\psi_{I}(x) u_{, x}^{I}+\phi_{J}(x) u^{J}+\psi_{J}(x) u_{, x}^{J},
$$




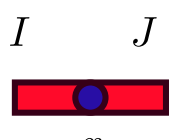

$x$

Support domain of FEM

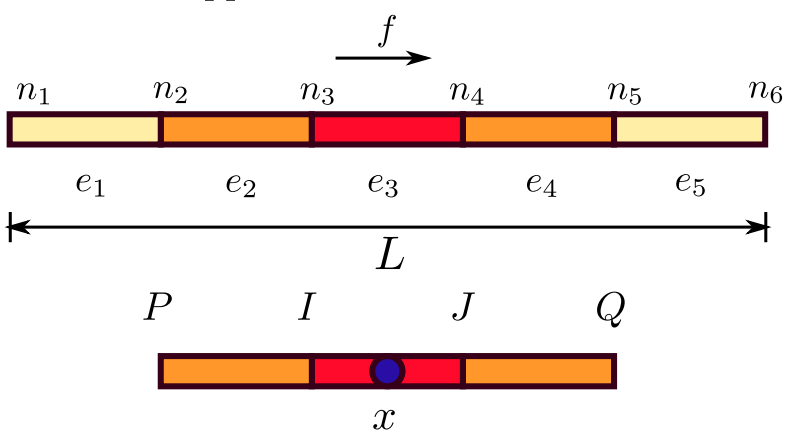

Support domain of DFEM

Figure 1: Discretization of the 1D domain and the element support domain of FEM and DFEM

where $u^{I}, u_{, x}^{I}$ denote the nodal displacement and nodal derivative of the displacement field at node $I$, respectively. $\ell=x_{J}-x_{I}$ is the length of the element. $\phi_{I}, \psi_{I}, \phi_{J}, \psi_{J}$ are the cubic Hermite basis polynomials given by:

$$
\begin{gathered}
\phi_{I}(x)=\left(1+2\left(\frac{x-x_{I}}{x_{J}-x_{I}}\right)\right)\left(\frac{x-x_{J}}{x_{J}-x_{I}}\right)^{2}, \\
\psi_{I}(x)=\left(x-x_{I}\right)\left(\frac{x-x_{J}}{x_{J}-x_{I}}\right)^{2}, \\
\phi_{J}(x)=\left(1-2\left(\frac{x-x_{J}}{x_{J}-x_{I}}\right)\right)\left(\frac{x-x_{I}}{x_{J}-x_{I}}\right)^{2}, \\
\psi_{J}(x)=\left(x-x_{J}\right)\left(\frac{x-x_{I}}{x_{J}-x_{I}}\right)^{2} .
\end{gathered}
$$

We note that

$$
\begin{array}{ll}
\phi_{I}\left(\mathbf{x}_{L}\right)=\delta_{I L}, & \phi_{I, x}\left(\mathbf{x}_{L}\right)=0 \\
\psi_{I}\left(\mathbf{x}_{L}\right)=0 \quad, & \psi_{I, x}\left(\mathbf{x}_{L}\right)=\delta_{I L},
\end{array}
$$

which guarantees the Dirichlet boundary conditions can be exactly applied in the second stage of interpolation. If we define the local coordinates as follows,

$$
L_{I}(x)=\frac{x-x_{J}}{\ell}, \quad L_{J}(x)=-\frac{x-x_{I}}{\ell},
$$

then the Hermite basis polynomials can be written as:

$$
\begin{gathered}
\phi_{I}(x)=L_{I}(x)+\left(L_{I}(x)\right)^{2} L_{J}(x)-L_{I}(x)\left(L_{J}(x)\right)^{2}, \\
\psi_{I}(x)=\ell L_{J}(x)\left(L_{I}(x)\right)^{2}, \\
\phi_{J}(x)=L_{J}(x)+\left(L_{J}(x)\right)^{2} L_{I}(x)-L_{J}(x)\left(L_{I}(x)\right)^{2}, \\
\psi_{J}(x)=-\ell L_{I}(x)\left(L_{J}(x)\right)^{2} .
\end{gathered}
$$


Subsequently, we will use the 'average' nodal gradients $\left(\bar{u}_{, x}^{I}, \bar{u}_{, x}^{J}\right)$ derived from finite element interpolation at each node to replace the gradients $\left(u_{, x}^{I}, u_{, x}^{J}\right)$ in Equation (1). But before we start calculating the average nodal gradients, an element set and a node set should be defined which closely relate to the derivation. First of all, we collect all the elements contained in the support domain* for a point of interest into the element set $\Lambda$. Then, all the support nodes for a point of interest are listed in the node set $\mathscr{N}$. For instance, in Figure 1, for the point of interest $x$ inside element $e_{3}, \Lambda=\left\{e_{3}\right\}$ and $\mathscr{N}=\left\{n_{3}, n_{4}\right\}$ (or $\mathscr{N}=\left\{x_{I}, x_{J}\right\}$ in a local representation) for classical FEM. While for nodes on the element boundary, like $n_{3}$ (or $x_{I}$ ), $\Lambda_{I}=\left\{e_{2}, e_{3}\right\}$ and $\mathscr{N}_{I}=\left\{n_{2}, n_{3}, n_{4}\right\}$ (or $\mathscr{N}_{I}=\left\{x_{P}, x_{I}, x_{J}\right\}$ ) for classical FEM. Now Equation (1) can be rewritten as:

$$
u^{h}(x)=\phi_{I}(x) u^{I}+\psi_{I}(x) \bar{u}_{, x}^{I}+\phi_{J}(x) u^{J}+\psi_{J}(x) \bar{u}_{, x}^{J}
$$

where

$$
\begin{gathered}
u^{I}=u\left(x_{I}\right)=N_{I}^{e_{3}}\left(x_{I}\right) u^{I}+N_{J}^{e_{3}}\left(x_{I}\right) u^{J}, \\
\bar{u}_{, x}^{I}=\bar{u}_{, x}\left(x_{I}\right)=\omega_{e_{2, I}} u_{, x}^{e_{2}}\left(x_{I}\right)+\omega_{e_{3, I}} u_{, x}^{e_{3}}\left(x_{I}\right),
\end{gathered}
$$

in which $N_{I}^{e_{3}}, N_{J}^{e_{3}}$ are linear finite element shape functions, ${ }^{\dagger} u_{, x}^{e_{2}}\left(x_{I}\right)$ is the nodal derivative at $x_{I}$ calculated in element $e_{2}$, which belongs to $\Lambda_{I}$, the support element set of $x_{I}$. $\omega_{e_{2, I}}$ denotes the weight of element $e_{2}$ in $\Lambda_{I}$. These parameters are calculated by:

$$
\begin{gathered}
u_{, x}^{e_{2}}\left(x_{I}\right)=N_{P, x}^{e_{2}}\left(x_{I}\right) u^{P}+N_{I, x}^{e_{2}}\left(x_{I}\right) u^{I}, \\
\omega_{e_{2, I}}=\frac{\operatorname{meas}\left(e_{2, I}\right)}{\operatorname{meas}\left(e_{2, I}\right)+\operatorname{meas}\left(e_{3, I}\right)},
\end{gathered}
$$

where $N_{P, x}^{e_{2}}(x), N_{I, x}^{e_{2}}(x)$ are the derivatives of the corresponding shape functions associated with element $e_{2}$. meas $(\cdot)$ denotes the length of the $1 \mathrm{D}$ element.

Substituting equations (10) and (9), into Equation (8) yields:

$$
\begin{aligned}
\bar{u}_{, x}^{I}=\bar{u}_{, x}\left(x_{I}\right)= & \omega_{e_{2, I}}\left(N_{P, x}^{e_{2}}\left(x_{I}\right) u^{P}+N_{I, x}^{e_{2}}\left(x_{I}\right) u^{I}\right)+ \\
& \omega_{e_{3, I}}\left(N_{I, x}^{e_{3}}\left(x_{I}\right) u^{I}+N_{J, x}^{e_{3}}\left(x_{I}\right) u^{J}\right),
\end{aligned}
$$

which can be rewritten as:

$$
\bar{u}_{, x}^{I}=\left[\begin{array}{lll}
\omega_{e_{2, I}} N_{P, x}^{e_{2}} & \omega_{e_{2, I}} N_{I, x}^{e_{2}}+\omega_{e_{3, I}} N_{I, x}^{e_{3}} & \omega_{e_{3, I}} N_{J, x}^{e_{3}}
\end{array}\right]\left[\begin{array}{c}
u^{P} \\
u^{I} \\
u^{J}
\end{array}\right] .
$$

By defining,

$$
\bar{N}_{L, x}\left(x_{I}\right)=\sum_{e_{i} \in \Lambda_{I}} \omega_{e_{i, I}} N_{L, x}^{e_{i}}\left(x_{I}\right), \quad L \in \mathscr{N}_{I},
$$

the averaged derivative at node $x_{I}$ can be written as

$$
\bar{u}_{, x}^{I}=\bar{u}_{, x}\left(x_{I}\right)=\bar{N}_{P, x}\left(x_{I}\right) u^{P}+\bar{N}_{I, x}\left(x_{I}\right) u^{I}+\bar{N}_{J, x}\left(x_{I}\right) u^{J} .
$$

\footnotetext{
*Support domain means the region for a point of interest $x$ in an element, where the shape functions are non-zero at $x$.

${ }^{\dagger}$ In order to emphasis the support domain of FEM, the element number is used as the superscript of the shape functions. In Equation (7), the displacement at $x_{I}$ (or $n_{3}$ ) is interpolated in the element of interest $e_{3}$, although $N_{J}^{e_{3}}\left(x_{I}\right)=0$, we still add this term for clarity.
} 
Now, substituting Equations (7) and (14) into (6) results in:

$$
\begin{aligned}
u^{h}(x)= & \phi_{I}(x)\left(N_{I}\left(x_{I}\right) u^{I}+N_{J}\left(x_{I}\right) u^{J}\right)+ \\
& \psi_{I}(x)\left(\bar{N}_{P, x}\left(x_{I}\right) u^{P}+\bar{N}_{I, x}\left(x_{I}\right) u^{I}+\bar{N}_{J, x}\left(x_{I}\right) u^{J}\right)+ \\
& \phi_{J}(x)\left(N_{I}\left(x_{J}\right) u^{I}+N_{J}\left(x_{J}\right) u^{J}\right)+ \\
& \psi_{J}(x)\left(\bar{N}_{I, x}\left(x_{J}\right) u^{I}+\bar{N}_{J, x}\left(x_{J}\right) u^{J}+\bar{N}_{Q, x}\left(x_{J}\right) u^{Q}\right) \\
= & \psi_{I}(x) \bar{N}_{P, x}\left(x_{I}\right) u^{P}+ \\
& \left(\phi_{I}(x) N_{I}\left(x_{I}\right)+\psi_{I}(x) \bar{N}_{I, x}\left(x_{I}\right)+\phi_{J}(x) N_{I}\left(x_{J}\right)+\psi_{J}(x) \bar{N}_{I, x}\left(x_{J}\right)\right) u^{I}+ \\
& \left(\phi_{I}(x) N_{J}\left(x_{I}\right)+\psi_{I}(x) \bar{N}_{J, x}\left(x_{I}\right)+\phi_{J}(x) N_{J}\left(x_{J}\right)+\psi_{J}(x) \bar{N}_{J, x}\left(x_{J}\right)\right) u^{J}+ \\
& \psi_{J}(x) \bar{N}_{Q, x}\left(x_{J}\right) u^{Q} .
\end{aligned}
$$

Hence, by defining,

$$
\hat{N}_{L}(x)=\phi_{I}(x) N_{L}\left(x_{I}\right)+\psi_{I}(x) \bar{N}_{L, x}\left(x_{I}\right)+\phi_{J}(x) N_{L}\left(x_{J}\right)+\psi_{J}(x) \bar{N}_{L, x}\left(x_{J}\right),
$$

the final form for the double-interpolation approximation can be obtained as:

$$
u^{h}(x)=\sum_{L \in \hat{\mathscr{N}}} \hat{N}_{L}(x) u^{L},
$$

in which $\hat{\mathscr{N}}$ denotes the support node set for the point of interest $x$ in DFEM. We also use $\hat{\Lambda}$ as the the support element set in DFEM. Thus, for the point of interest $x, \hat{\Lambda}=\Lambda_{I} \cup$ $\Lambda_{J}=\left\{e_{2}, e_{3}, e_{4}\right\}, \hat{\mathscr{N}}=\mathscr{N}_{I} \cup \mathscr{N}_{J}=\left\{n_{2}, n_{3}, n_{4}, n_{5}\right\}$ (or $\hat{\mathscr{N}}=\left\{x_{P}, x_{I}, x_{J}, x_{Q}\right\}$ in the local representation as presented in Figure 1). Due to the computation of $\bar{u}_{x x}^{I}$ and $\bar{u}_{, x}^{J}$, the support domain of point of interest $x$ in $e_{3}$ has been expanded in the DFEM approximation. Similarly, the support domain of element boundary node $n_{3}$ (or $x_{I}$ ) is also larger in DFEM, i.e., $\hat{\Lambda}_{I}=$ $\left\{e_{1}, e_{2}, e_{3}, e_{4}\right\}$ and $\hat{\mathscr{N}}_{I}=\left\{n_{1}, n_{2}, n_{3}, n_{4}, n_{5}\right\}$. It can be observed that derivative interpolants are embedded into Equation 17. We can also infer that due to the enlargement of the local support domain, DFEM will result in an increased bandwidth, thus have an increased computational cost per DOF, but this is essential to construct the $C^{1}$ interpolants. Figure 2 shows the DFEM shape function and derivative at node $n_{3}$.

To more clearly depict the behavior of the proposed method, a numerical example is considered in the following discussion. For this purpose a 1D bar of Young's modulus $E$, cross section $A$ and length $L$ (as illustrated in Figure 1) problem is solved using both DFEM and FEM. The governing equations for the $1 \mathrm{D}$ problem are given by:

$$
\begin{aligned}
& \text { the equilibium equation, } E A \frac{\mathrm{d}^{2} u}{\mathrm{~d} x^{2}}+f=0, \\
& \text { the strain displacement relation, } \quad \epsilon(x)=u_{, x}(x), \\
& \text { the constitutive law, } \sigma(x)=E \epsilon_{, x}(x), \\
& \text { boundary condition, }\left.\quad u\right|_{x=0}=0 \text {, and }\left.\quad \sigma\right|_{x=L}=0,
\end{aligned}
$$

where $f$ is a uniform body force applied to the $1 \mathrm{D}$ bar. The exact solution for the displacement and stress are given by:

$$
\begin{gathered}
u(x)=\frac{f L^{2}}{E A}\left(\frac{x}{L}-\frac{1}{2}\left(\frac{x}{L}\right)^{2}\right), \\
\sigma(x)=\frac{f L}{A}\left(1-\frac{x}{L}\right) .
\end{gathered}
$$




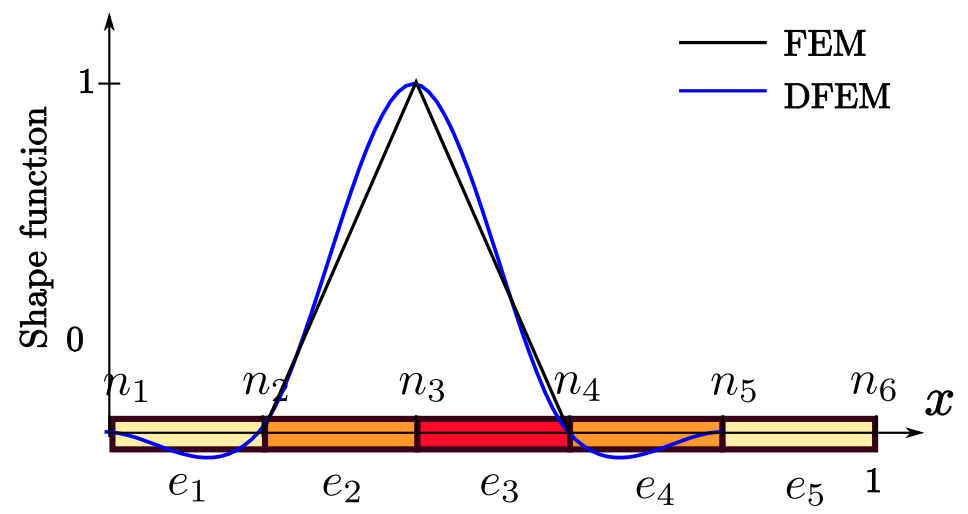

(a)

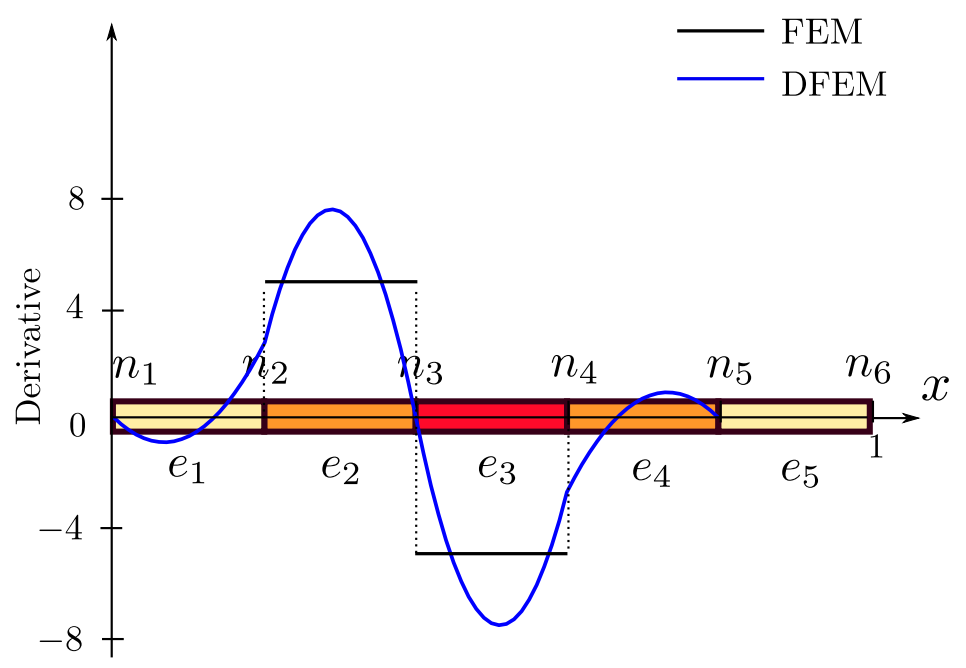

(b)

Figure 2: The 1D DFEM shape function and its derivative at node 3. Note that the shape functions do not satisfy the positivity condition, but do provide the Kronecker Delta property 
For simplicity, all these parameters are assumed to have unit value in the simulation.

Figure 3 compares the displacement and stress values obtained by both FEM and DFEM. It can be observed from the figure that DFEM captures the exact stress solution much better than FEM. The deterioration of the DFEM solution near the boundary nodes is attributed to the automatic recovery of the nodal gradients at the end points, which will be explained in the following section. Figure 4 plots the relative error in the displacement and energy norm of the 1D bar problem (The definitions of these norms are given in section 4). It is clearly illustrated that the DFEM approximation in 1D achieves at a rate comprised between the optimal rate of convergence for linear and quadratic complete approximation.

\section{$2.22 \mathrm{D}$ approximation by double interpolation}

As illustrated in Figure $5, \mathbf{x}=(x, y)$ denotes the point of interest in triangle $I J K$. Analogous to the derivation for the $1 \mathrm{D}$ formulation, the $2 \mathrm{D}$ double-interpolation approximation in a mesh of triangular element can be cosntructed as follows:

$$
\begin{gathered}
\mathbf{u}^{h}(\mathbf{x})=\sum_{L \in \hat{N}} \hat{N}_{L}(\mathbf{x}) \mathbf{u}^{L} \\
\hat{N}_{L}(\mathbf{x})=\phi_{I}(\mathbf{x}) N_{L}\left(\mathbf{x}_{I}\right)+\psi_{I}(\mathbf{x}) \bar{N}_{L, x}\left(\mathbf{x}_{I}\right)+\varphi_{I}(\mathbf{x}) \bar{N}_{L, y}\left(\mathbf{x}_{I}\right)+ \\
\phi_{J}(\mathbf{x}) N_{L}\left(\mathbf{x}_{J}\right)+\psi_{J}(\mathbf{x}) \bar{N}_{L, x}\left(\mathbf{x}_{J}\right)+\varphi_{J}(\mathbf{x}) \bar{N}_{L, y}\left(\mathbf{x}_{J}\right)+ \\
\phi_{K}(\mathbf{x}) N_{L}\left(\mathbf{x}_{K}\right)+\psi_{K}(\mathbf{x}) \bar{N}_{L, x}\left(\mathbf{x}_{K}\right)+\varphi_{K}(\mathbf{x}) \bar{N}_{L, y}\left(\mathbf{x}_{K}\right),
\end{gathered}
$$

where $\mathbf{u}^{L}$ is the nodal displacement vector. In the following discussion the evaluation of the average derivative of the shape function at node $\mathbf{x}_{I}$ is considered. The average derivative of the shape function at node $\mathbf{x}_{I}$ can be written as:

$$
\begin{aligned}
& \bar{N}_{L, x}\left(\mathbf{x}_{I}\right)=\sum_{e_{i, I} \in \Lambda_{I}} \omega_{e_{i, I}} N_{L, x}^{e_{i}}\left(\mathbf{x}_{I}\right), \\
& \bar{N}_{L, y}\left(\mathbf{x}_{I}\right)=\sum_{e_{i, I} \in \Lambda_{I}} \omega_{e_{i, I}} N_{L, y}^{e_{i}}\left(\mathbf{x}_{I}\right),
\end{aligned}
$$

where $\omega_{e_{i, I}}$ is the weight of element $e_{i}$ in $\Lambda_{I}$ and is computed by:

$$
\omega_{e_{i, I}}=\operatorname{meas}\left(e_{i}\right) / \sum_{e_{i} \in \Lambda_{I}} \operatorname{meas}\left(e_{i}\right) .
$$

Here meas $(\cdot)$ denotes the area of a triangular element. An example of how to evaluate the weight of an element is presented in Figure 5. $\phi_{I}, \psi_{I}$ and $\varphi_{I}$ form the polynomial basis associated with $\mathbf{x}_{I}$, which satisfies the following interpolating conditions:

$$
\begin{array}{lll}
\phi_{I}\left(\mathbf{x}_{L}\right)=\delta_{I L}, & \phi_{I, x}\left(\mathbf{x}_{L}\right)=0 \quad, & \phi_{I, y}\left(\mathbf{x}_{L}\right)=0 \\
\psi_{I}\left(\mathbf{x}_{L}\right)=0 & , \quad \psi_{I, x}\left(\mathbf{x}_{L}\right)=\delta_{I L}, & \psi_{I, y}\left(\mathbf{x}_{L}\right)=0 \\
\varphi_{I}\left(\mathbf{x}_{L}\right)=0 \quad, \quad \varphi_{I, x}\left(\mathbf{x}_{L}\right)=0 \quad, & \varphi_{I, y}\left(\mathbf{x}_{L}\right)=\delta_{I L}
\end{array}
$$

And these polynomial basis functions are given by:

$$
\begin{aligned}
\phi_{I}(\mathbf{x})= & L_{I}(\mathbf{x})+\left(L_{I}(\mathbf{x})\right)^{2} L_{J}(\mathbf{x})+\left(L_{I}(\mathbf{x})\right)^{2} L_{K}(\mathbf{x}) \\
& -L_{I}(\mathbf{x})\left(L_{J}(\mathbf{x})\right)^{2}-L_{I}(\mathbf{x})\left(L_{K}(\mathbf{x})\right)^{2},
\end{aligned}
$$




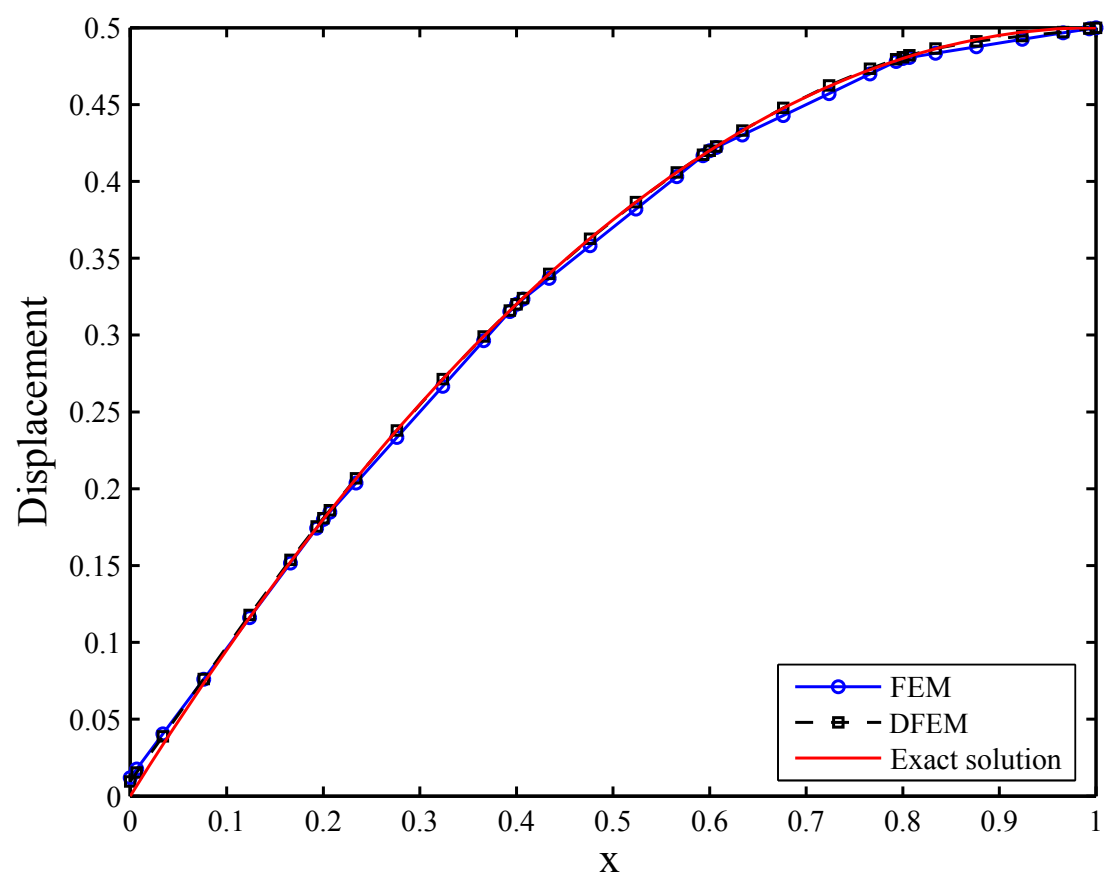

(a)

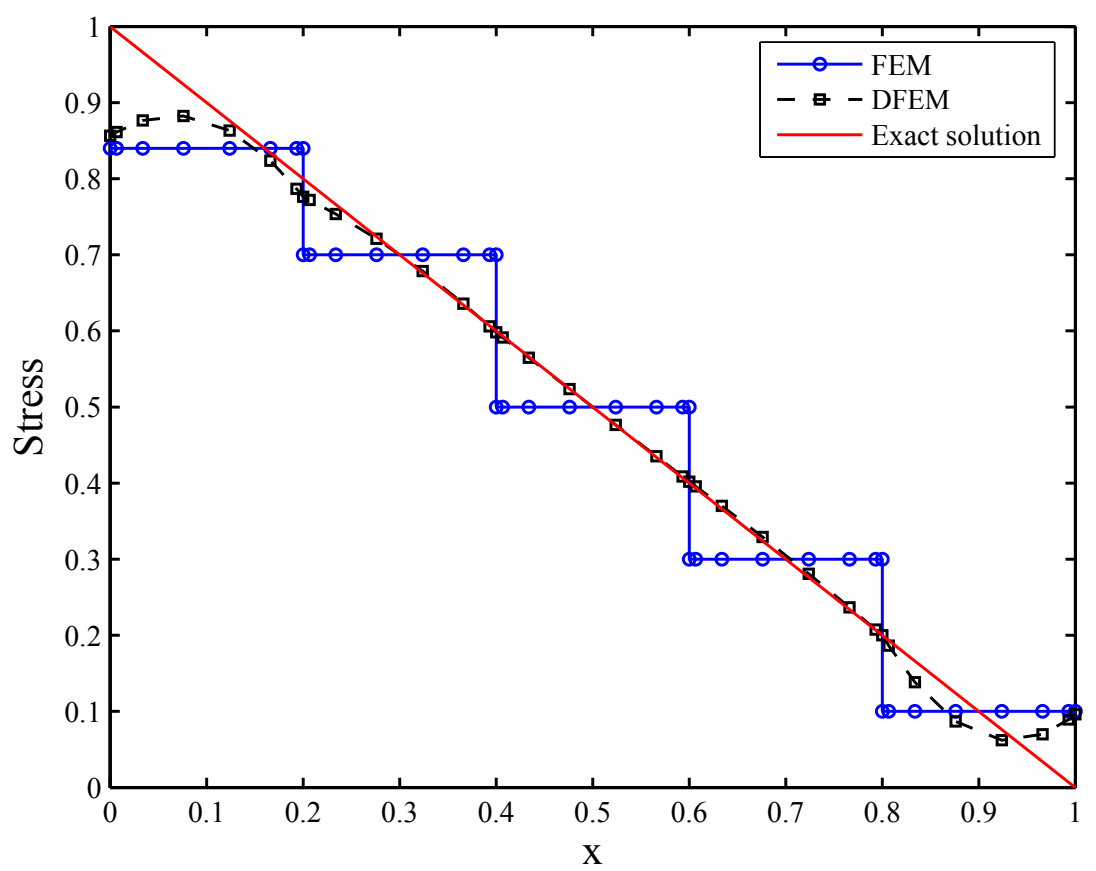

(b)

Figure 3: The comparison of FEM and DFEM results for the 1D bar example:(a)displacement;(b)stress. Note that superior stress accuracy provided by DFEM 


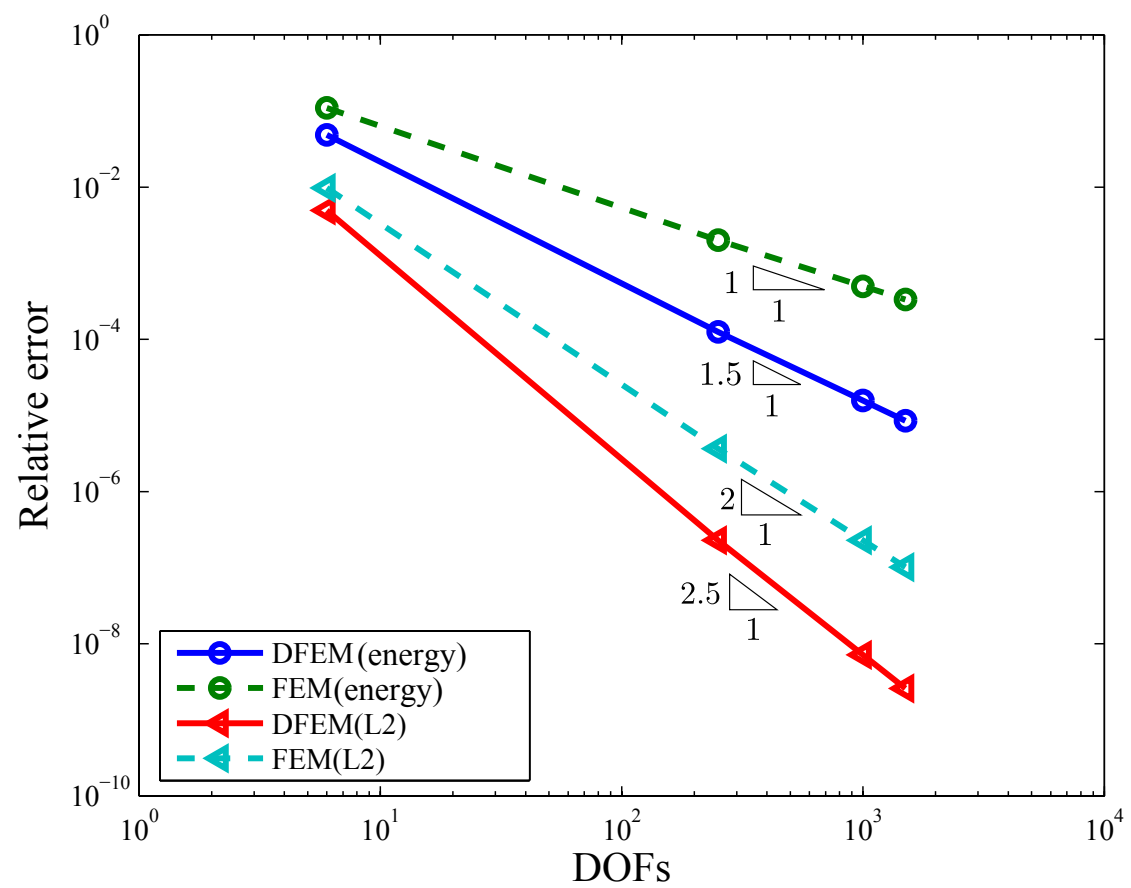

Figure 4: Relative error in displacement and energy norm for the 1D bar problem

- - Support nodes of DFEM

- Support nodes of FEM

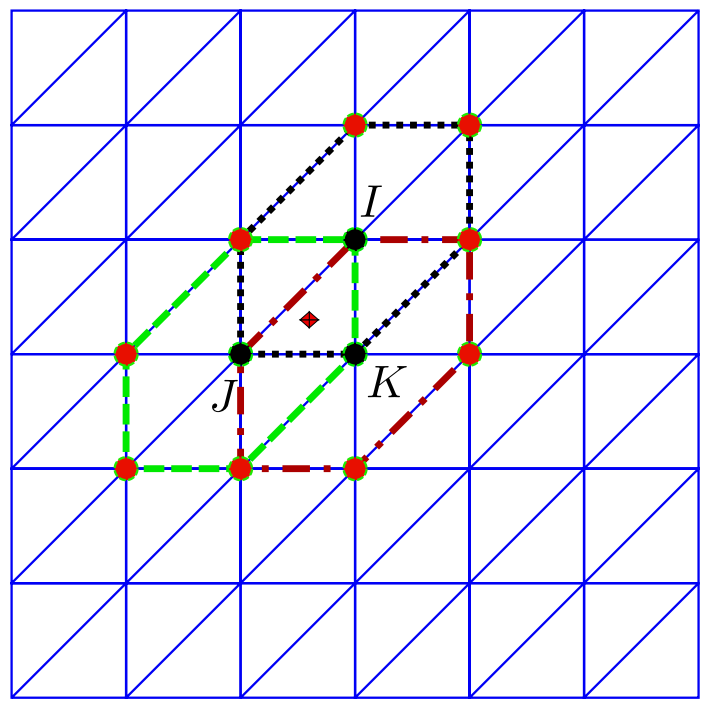

$\Lambda_{I}$ : support domain of node $I$

$=-=-\Lambda_{J}:$ support domain of node $J$

- - " $\Lambda_{K}$ : support domain of node $K$

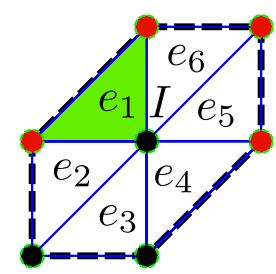

$\omega_{e_{1}}=S_{e_{1}} /\left(\sum_{e_{i} \in \Lambda_{I}} S_{e_{i}}\right)$

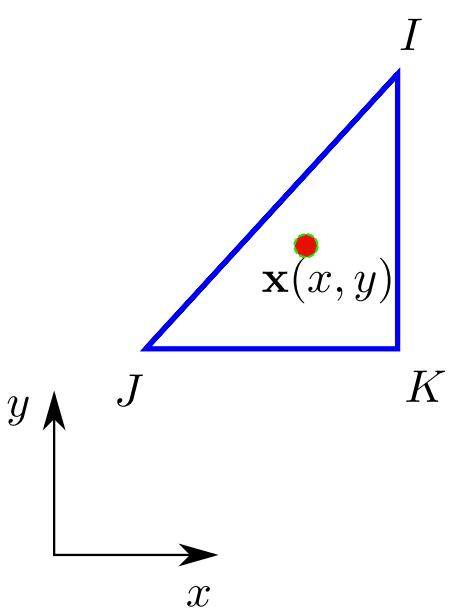

Figure 5: Illustration for the support domain of DFEM 


$$
\begin{aligned}
\psi_{I}(\mathbf{x})=- & c_{J}\left(L_{K}(\mathbf{x})\left(L_{I}(\mathbf{x})\right)^{2}+\frac{1}{2} L_{I}(\mathbf{x}) L_{J}(\mathbf{x}) L_{K}(\mathbf{x})\right)+ \\
& c_{K}\left(\left(L_{I}(\mathbf{x})\right)^{2} L_{J}(\mathbf{x})+\frac{1}{2} L_{I}(\mathbf{x}) L_{J}(\mathbf{x}) L_{K}(\mathbf{x})\right) \\
\varphi_{I}(\mathbf{x})= & b_{J}\left(L_{K}(\mathbf{x})\left(L_{I}(\mathbf{x})\right)^{2}+\frac{1}{2} L_{I}(\mathbf{x}) L_{J}(\mathbf{x}) L_{K}(\mathbf{x})\right)- \\
& b_{K}\left(\left(L_{I}(\mathbf{x})\right)^{2} L_{J}(\mathbf{x})+\frac{1}{2} L_{I}(\mathbf{x}) L_{J}(\mathbf{x}) L_{K}(\mathbf{x})\right) .
\end{aligned}
$$

Note that the polynomial basis functions $\phi_{J}, \psi_{J}, \varphi_{J}, \phi_{K}, \psi_{K}$ and $\varphi_{K}$ can be obtained by the above definitions via cyclic permutation of indices $I, J$ and $K$. In the above equations, $L_{I}, L_{J}$ and $L_{K}$ are the area coordinates of the point of interest $\mathbf{x}$ in triangle $I J K$. For the point of interest $\mathbf{x}$ in Figure 5, the $L_{I}, a_{I}, b_{I}$ and $c_{I}$ are presented as follows:

$$
\begin{gathered}
L_{I}(\mathbf{x})=\frac{1}{2 \triangle}\left(a_{I}+b_{I} x+c_{I} y\right), \\
a_{I}=x_{J} y_{K}-x_{K} y_{J}, \\
b_{I}=y_{J}-y_{K}, \\
c_{I}=x_{K}-x_{J},
\end{gathered}
$$

where $\triangle$ is the area of triangle $I J K$. Further, $L_{J}, L_{K}, a_{J}, b_{J}, b_{K}, a_{I}, c_{J}$ and $c_{K}$ can be obtained using the above definitions via cyclic permutations of indices $I, J$ and $K$.

When the point of interest lies on one of the edges, for example on edge $I J$, the basis functions will boil down to 1D basis functions and will be consistent with the 1D form presented in the preceding section.

The DFEM shape functions posess the properties such as linear completeness, partition of unity and Kronecker delta property [49]. In addition, the 2D DFEM possesses $C^{1}$ continuity at the nodes and $C^{0}$ continuity on edges. Compared to 3 -noded triangular elements, the DFEM basis functions can achieve a higher-order convergence rate without the introduction of additional nodes, which will be seen the numerical examples in the next section. However, this attractive feature comes with the price of an increased bandwidth as the neighboring nodes are used to obtain the nodal gradients necessary for the second interpolation. The details of such computational costs will be discussed in the section devoted to numerical examples.

\subsection{Modification of the nodal gradients}

When $C^{0}$ continuity of the primal field at a node is needed, for instance on the nodes along a material interface, it is useful to modify the calculation of the average nodal gradient as discussed below. The calculation of the nodal gradient can be performed as follows:

$$
\bar{N}_{L, x}\left(\mathbf{x}_{I}\right)=N_{L, x}^{e}\left(\mathbf{x}_{I}\right) \text {. }
$$

The right hand side is the derivative of $N_{L}$ computed in element $e$, in which the point of interest $\mathbf{x}$ is located. This is easily done in the implementation by replacing the average derivative with the derivative in the element of interest. It can be observed that nodes at the endpoint of a 1D bar automatically satisfy the above equation. All the topological enriched nodes in XFEM (the nodes circled by red boxes in Figure 7 and Figure 8) have been relaxed to $C^{0}$ as well due to the fact that during the calculation of average gradients in Equation (22), the contribution from split elements cannot be computed directly as from continuous elements in an area weighted manner (Equation (23)) due to the discontinuity. This is similar to difficulties encountered in smoothing enriched approximations [9][10]. 


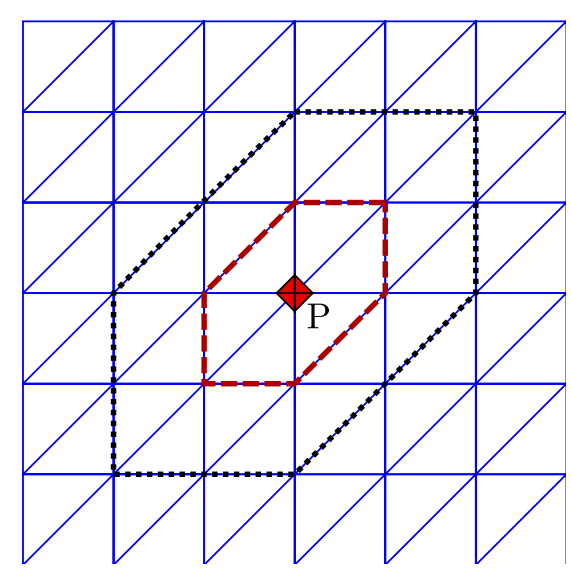

Support domain of DFEM

--_- Support domain of FEM

(a)

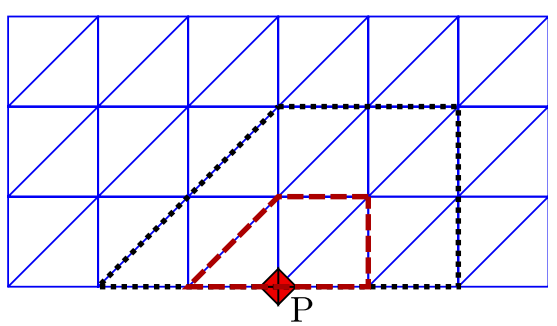

........ Support domain of DFEM

----- Support domain of FEM

(c)

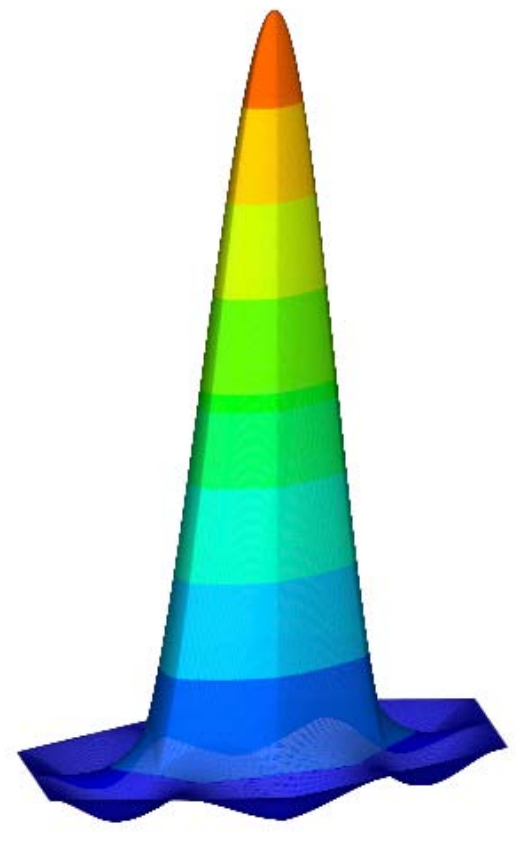

$$
\hat{N}_{P}(\mathbf{x})
$$

$1.000 \mathrm{E}+00$ $8.750 \mathrm{E}-01$

7.500E- 01

$6.250 \mathrm{E}-01$

$5.000 \mathrm{E}-01$

4.753E- -01

3.750E-01

$2.500 \mathrm{E}-01$

$1.250 \mathrm{E}-01$

$0.000 \mathrm{E}+00$

(b)

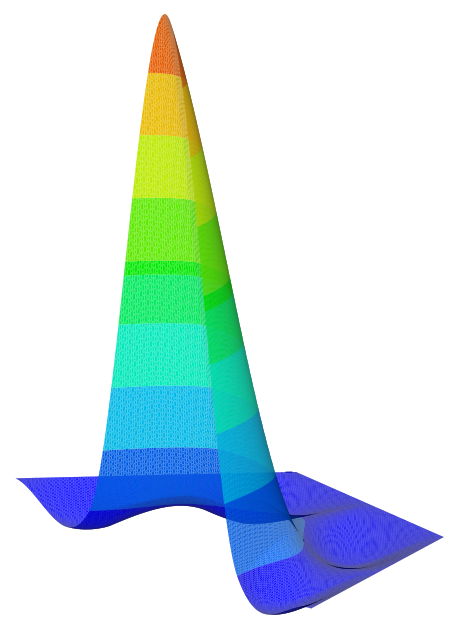

$\hat{N}_{P}(\mathbf{x})$

$1.000 \mathrm{E}+00$

8.750E-01

$7.500 \mathrm{E}-0$

$6.250 \mathrm{E}-01$

$5.000 \mathrm{E}-01$

$4.719 \mathrm{E}-01$

$3.750 \mathrm{E}-0$

$2.500 \mathrm{E}-0$

$1.250 \mathrm{E}-0$

$0.000 \mathrm{E}+00$

(d)

Figure 6: The shape functions of DFEM in 2D 


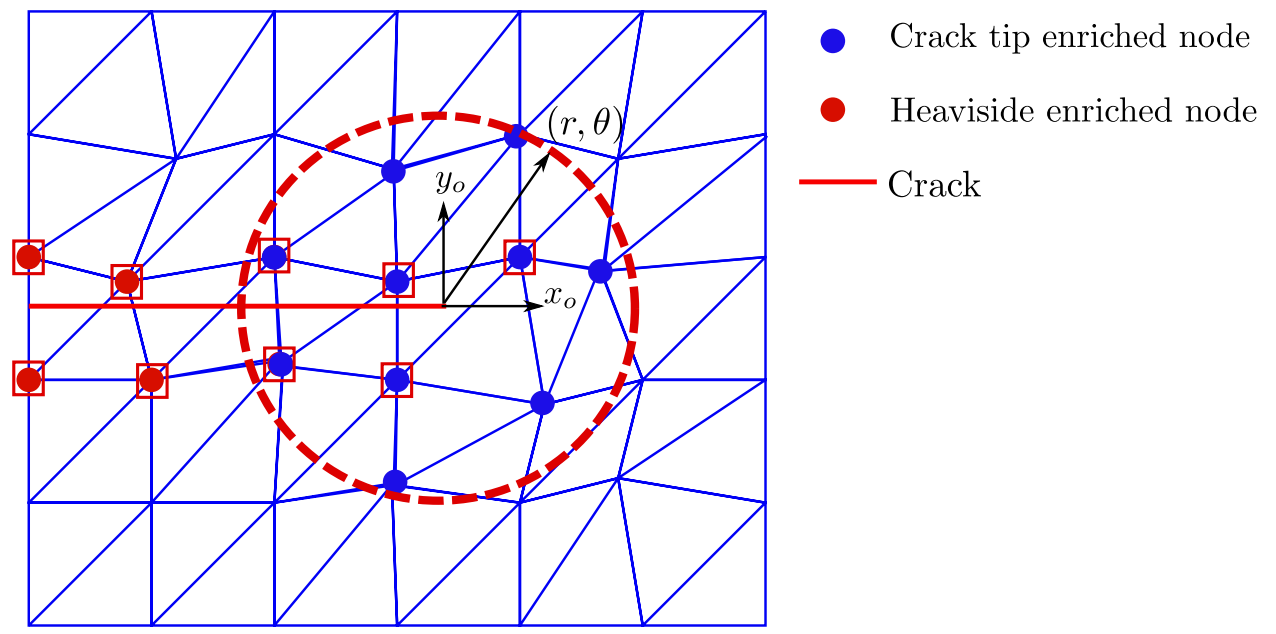

Figure 7: Nodal enrichment in XDFEM; the nodes encircled by red box are degenerated to $C^{0}$, see section 2.3

\subsection{The enriched 2D double-interpolation approximation}

The extended finite element method (XFEM) uses a partition of unity which allows for the addition of a priori knowledge about the solution of boundary value problems into the approximation space of the numerical solution. The crack can be described in XFEM by enriching the standard displacement approximation as follows:

$$
\mathbf{u}^{h}(\mathbf{x})=\sum_{I \in \mathscr{N}_{I}} \hat{N}_{I}(\mathbf{x}) \mathbf{u}^{I}+\sum_{J \in \mathscr{N}_{J}} \hat{N}_{J}(\mathbf{x}) H(\mathbf{x}) \mathbf{a}^{J}+\sum_{K \in \mathscr{N}_{K}} \hat{N}_{K}(\mathbf{x}) \sum_{\alpha=1}^{4} f_{\alpha}(\mathbf{x}) \mathbf{b}^{K \alpha},
$$

where $\mathbf{u}^{I}$ are the regular DOFs, $\mathbf{a}^{J}$ are the additional Heaviside enriched DOFs, and $\mathbf{b}^{K \alpha}$ are the additional crack tip enriched DOFs. $\mathscr{N}_{I}, \mathscr{N}_{J}$ and $\mathscr{N}_{K}$ are the collections of regular nonenriched nodes, Heaviside enriched nodes and crack tip enriched nodes, respectively. $H(\cdot)$ is the Heaviside function. The crack tip enrichment functions are defined as:

$$
\left\{f_{\alpha}(r, \theta), \alpha=1,4\right\}=\left\{\sqrt{r} \sin \frac{\theta}{2}, \sqrt{r} \cos \frac{\theta}{2}, \sqrt{r} \sin \frac{\theta}{2} \sin \theta, \sqrt{r} \cos \frac{\theta}{2} \sin \theta\right\},
$$

where $(r, \theta)$ are the polar coordinates of the crack tip (Figure 7). Figure 9 compares the Heaviside enriched shape functions obtained with XFEM and XDFEM which are defined in Figure 8.

\section{Weak form and discretized formulations}

For an elastic body as in Figure 10 defined by Hooke's tensor $\mathbf{C}$ and undergoing small strains and small displacements, the equilibrium equations and boundary conditions for the Cauchy stress $\boldsymbol{\sigma}$ and the displacement field $\mathbf{u}$ write:

$$
\begin{array}{ccc}
\nabla \cdot \boldsymbol{\sigma}=\mathbf{0} & \text { in } & \Omega, \\
\boldsymbol{\sigma} \cdot \mathbf{n}=\overline{\mathbf{t}} & \text { on } & \Gamma_{t} \\
\mathbf{u}=\overline{\mathbf{u}} & \text { on } & \Gamma_{u} .
\end{array}
$$




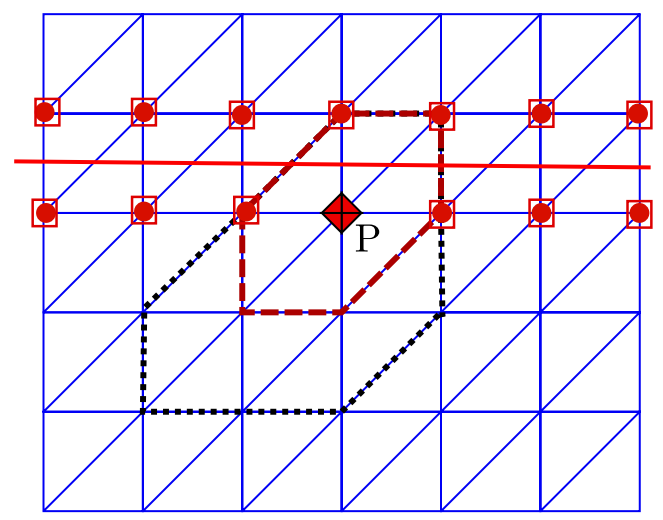

\section{.......... Support domain of DFEM \\ ----- Support domain of FEM Crack \\ - Heaviside enriched node}

Figure 8: The support domain of enriched DFEM; the nodes encircled by red box are degenerated to $C^{0}$, see section 2.3

Here $\overline{\mathbf{t}}$ is the traction imposed on boundary $\Gamma_{t}$. Further, assuming traction free crack faces:

$$
\boldsymbol{\sigma} \cdot \mathbf{n}=\mathbf{0} \quad \text { on } \quad \Gamma_{c^{+}} \text {and } \Gamma_{c^{-}},
$$

where $\Gamma_{c^{+}}, \Gamma_{c^{-}}$are the upper and lower crack surfaces respectively. The strain-displacement relation and the constitutive law are respectively as:

$$
\begin{gathered}
\boldsymbol{\epsilon}=\frac{1}{2}\left(\nabla+\nabla^{\mathrm{T}}\right) \otimes \mathbf{u}, \\
\boldsymbol{\sigma}=\mathbf{C}: \boldsymbol{\epsilon} .
\end{gathered}
$$

Using a Bubnov-Galerkin weighted residual formulation based on Lagrange test and trial spaces, substituting the trial and test functions into the weak form of Equation (30), and using the arbitrariness of nodal variations, the discretized equations can be written:

$$
\mathbf{K u}=\mathbf{f},
$$

where $\mathbf{u}$ is the nodal vector of the unknown displacements and $\mathbf{K}$ is the stiffness matrix. The elemental form of $\mathbf{K}$ for element $e$ is given by:

$$
\mathbf{K}_{I J}^{e}=\left[\begin{array}{lll}
\mathbf{K}_{I J}^{u u} & \mathbf{K}_{I J}^{u a} & \mathbf{K}_{I J}^{u b} \\
\mathbf{K}_{I J}^{a u} & \mathbf{K}_{I J}^{a a} & \mathbf{K}_{I J}^{a b} \\
\mathbf{K}_{I J}^{b u} & \mathbf{K}_{I J}^{b a} & \mathbf{K}_{I J}^{b b}
\end{array}\right] .
$$

The external force vector $\mathbf{f}$ is defined as

$$
\mathbf{f}_{I}=\left\{\begin{array}{llllll}
\mathbf{f}_{I}^{u} & \mathbf{f}_{I}^{a} & \mathbf{f}_{I}^{b^{1}} & \mathbf{f}_{I}^{b^{2}} & \mathbf{f}_{I}^{b^{3}} & \mathbf{f}_{I}^{b^{4}}
\end{array}\right\} .
$$

The submatrices and vectors in Equations (34) and (35) are:

$$
\begin{gathered}
\mathbf{K}_{I J}^{r s}=\int_{\Omega^{e}}\left(\mathbf{B}_{I}^{r}\right)^{T} \mathbf{C B}_{J}^{s} \mathrm{~d} \Omega \quad(r, s=u, a, b), \\
\mathbf{f}_{I}^{u}=\int_{\partial \Omega_{t}^{h} \cap \partial \Omega^{e}} \hat{N}_{I} \overline{\mathbf{t}} \mathrm{d} \Gamma \\
\mathbf{f}_{I}^{a}=\int_{\partial \Omega_{t}^{h} \cap \partial \Omega^{e}} \hat{N}_{I} H \overline{\mathbf{t}} \mathrm{d} \Gamma
\end{gathered}
$$




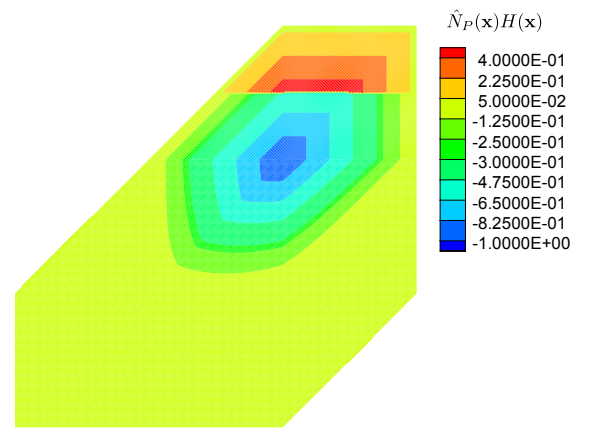

(a) XDFEM

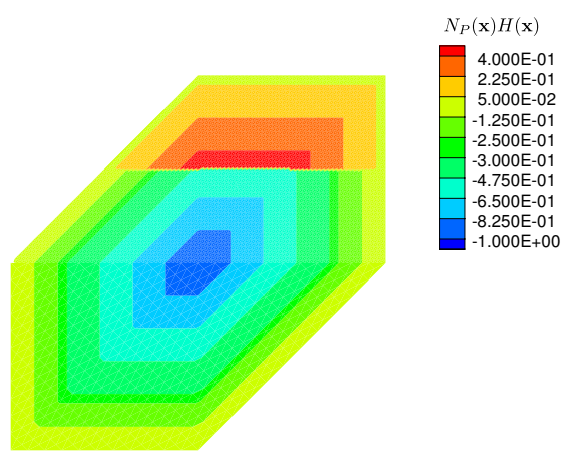

(c) XFEM

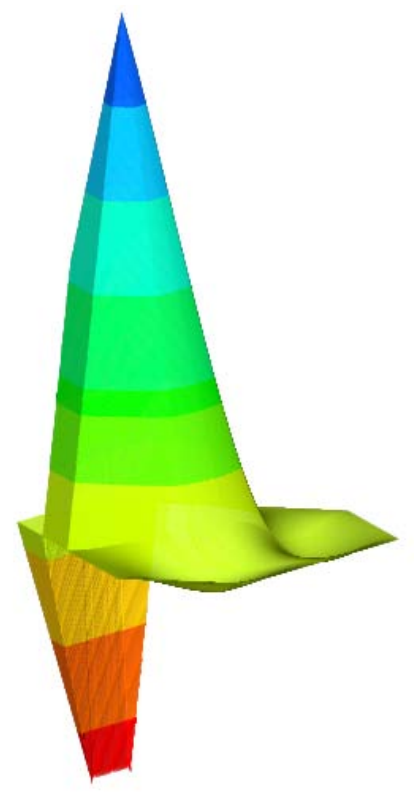

$\hat{N}_{P}(\mathbf{x}) H(\mathbf{x})$

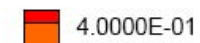

2.2500E-01

5.0000E-02

$-1.2500 \mathrm{E}-01$

$-2.5000 \mathrm{E}-01$

-3.0000 E-01

-4.7500 E-01

$-6.5000 \mathrm{E}-01$

$-8.2500 \mathrm{E}-01$

$-1.0000 E+00$

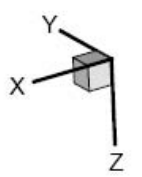

(b) XDFEM

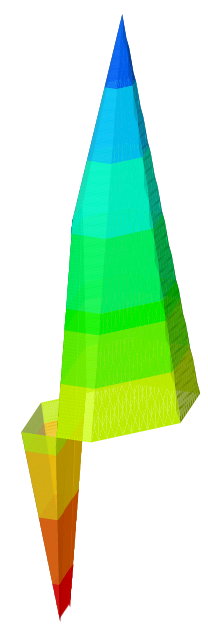

$N_{P}(\mathbf{x}) H(\mathbf{x})$

$4.000 \mathrm{E}-01$
$2.200 \mathrm{E}-01$

$4.000 \mathrm{E}-01$
$2.250 \mathrm{E}-01$
$5.000 \mathrm{E}-02$

$5.000 \mathrm{E}-02$
$-1.250 \mathrm{E}-01$

$-2.500 \mathrm{E}-01$

$-3.000 \mathrm{E}-01$

$-4.750 \mathrm{E}-01$

$-6.500 \mathrm{E}-01$

$-8.250 \mathrm{E}-01$

$-1.000 \mathrm{E}+0$

$\left.7\right|_{z}$

(d) XFEM

Figure 9: Contour plot of Heaviside enriched shape functions 


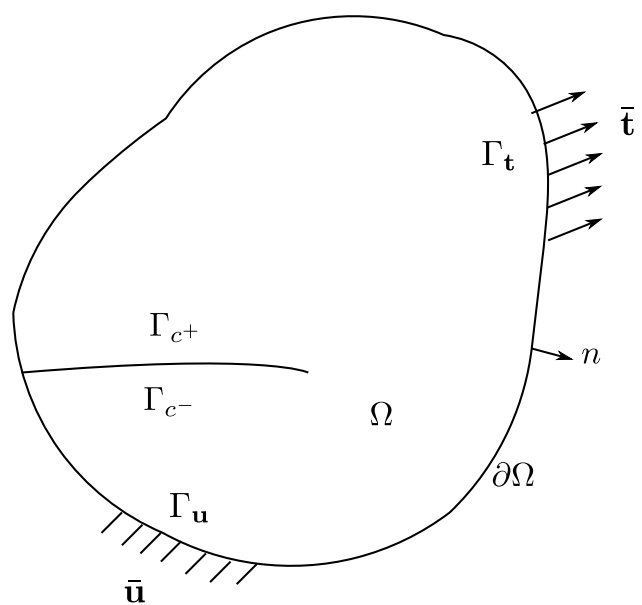

Figure 10: Elastic body with a crack, $\partial \Omega=\Gamma_{\mathbf{u}} \cup \Gamma_{\mathbf{t}}, \Gamma_{\mathbf{u}} \cap \Gamma_{\mathbf{t}}=\emptyset$

$$
\mathbf{f}_{I}^{b^{\alpha}}=\int_{\partial \Omega_{t}^{h} \cap \partial \Omega^{e}} \hat{N}_{I} f_{\alpha} \overline{\mathbf{t}} \mathrm{d} \Gamma \quad(\alpha=1,2,3,4) .
$$

In Equation (36a), $\mathbf{B}_{I}^{u}, \mathbf{B}_{I}^{a}$ and $\mathbf{B}_{I}^{b^{\alpha}}$ are given by

$$
\begin{aligned}
& \mathbf{B}_{I}^{u}=\left[\begin{array}{cc}
\hat{N}_{I, x} & 0 \\
0 & \hat{N}_{I, y} \\
\hat{N}_{I, y} & \hat{N}_{I, x}
\end{array}\right] \\
& \mathbf{B}_{I}^{a}=\left[\begin{array}{cc}
\left(\hat{N}_{I}\left(H-H_{I}\right)\right)_{, x} & 0 \\
0 & \left(\hat{N}_{I}\left(H-H_{I}\right)\right)_{, y} \\
\left(\hat{N}_{I}\left(H-H_{I}\right)\right)_{, y} & \left(\hat{N}_{I}\left(H-H_{I}\right)_{, x}\right.
\end{array}\right], \\
& \mathbf{B}_{I}^{b}=\left[\begin{array}{llll}
\mathbf{B}_{I}^{b^{1}} & \mathbf{B}_{I}^{b^{2}} & \mathbf{B}_{I}^{b^{3}} & \mathbf{B}_{I}^{b^{4}}
\end{array}\right], \\
& \mathbf{B}_{I}^{b^{\alpha}}=\left[\begin{array}{cc}
\left(\hat{N}_{I}\left(f_{\alpha}-f_{\alpha I}\right)\right)_{, x} & 0 \\
0 & \left(\hat{N}_{I}\left(f_{\alpha}-f_{\alpha I}\right)\right)_{, y} \\
\left(\hat{N}_{I}\left(f_{\alpha}-f_{\alpha I}\right)\right)_{, y} & \left(\hat{N}_{I}\left(f_{\alpha}-f_{\alpha I}\right)\right)_{, x}
\end{array}\right] \quad(\alpha=1-4) .
\end{aligned}
$$

In order to obtain the nodal displacements in a more straightforward manner, the shifted-basis is adopted in the above equations. More details regarding XFEM implementation can be found in [13].

\section{Numerical examples}

A set of numerical examples is chosen to assess the efficiency and usefulness of the doubleinterpolation and its enriched form. In order to assess the convergence rate of each method, the relative error measured in the displacement $L_{2}$ norm and the energy norm are defined, respectively, as:

$$
R_{d}=\sqrt{\frac{\int_{\Omega}\left(\mathbf{u}^{h}-\mathbf{u}\right)^{\mathrm{T}}\left(\mathbf{u}^{h}-\mathbf{u}\right) \mathrm{d} \Omega}{\int_{\Omega} \mathbf{u}^{\mathrm{T}} \mathbf{u} \mathrm{d} \Omega}},
$$




$$
R_{e}=\sqrt{\frac{\int_{\Omega}\left(\boldsymbol{\sigma}^{h}-\boldsymbol{\sigma}\right)^{\mathrm{T}}\left(\boldsymbol{\epsilon}^{h}-\boldsymbol{\epsilon}\right) \mathrm{d} \Omega}{\int_{\Omega} \boldsymbol{\sigma}^{\mathrm{T}} \boldsymbol{\epsilon} \Omega} .}
$$

where, the fields with superscript ' $h$ ' refer to the approximation, and $\boldsymbol{\sigma}, \boldsymbol{\epsilon}, \mathbf{u}$ are exact fields. Unless specified otherwise, the Young's modulus E and Poisson's ratio $\nu$ are assumed to be 1000 and 0.3 respectively. The constants $\mu$ and $\kappa$ are given by:

$$
\begin{gathered}
\mu=\frac{E}{2(1+\nu)}, \\
\kappa= \begin{cases}3-4 \nu, & \text { Plane strain } \\
(1-\nu) /(3+\nu), & \text { Plane stress }\end{cases}
\end{gathered}
$$

\subsection{Higher-order convergence test}

The first example will investigate the precision and convergence rate of DFEM in comparison with the 3-noded triangular element (T3) and 6-noded triangular element (T6) by solving the Laplace equation:

$$
\begin{gathered}
-\Delta u=f, \quad \text { in } \Omega, \\
u=\bar{u}=0, \quad \text { on } \partial \Omega .
\end{gathered}
$$

where $\Delta$ is the Laplace operator, $u$ the scalar primary variable and $f$ the source term. Here the domain $\Omega$ is selected as a square with dimensions $[-1,1] \times[-1,1] \subset \mathbb{R}^{2}$. And $f$ is given as:

$$
f(x, y)=5 \pi^{2} \sin (2 \pi x) \sin (\pi y) .
$$

The analytical solution of $u$ and its derivatives $u_{, x}, u_{, y}$ can be written as:

$$
\begin{gathered}
u(x, y)=\sin (2 \pi x) \sin (\pi y), \\
u_{, x}(x, y)=2 \pi \cos (2 \pi x) \sin (\pi y), \\
u_{, y}(x, y)=\pi \sin (2 \pi x) \cos (\pi y) .
\end{gathered}
$$

And errors in the $L_{2}$ and energy norm of the primary variable are defined as:

$$
\begin{gathered}
R_{d}=\sqrt{\frac{\int_{\Omega}\left(u^{h}-u\right)^{2} \mathrm{~d} \Omega}{\int_{\Omega} u^{2} \mathrm{~d} \Omega}}, \\
R_{e}=\sqrt{\frac{\int_{\Omega}\left(\nabla u^{h}-\nabla u\right)^{\mathrm{T}}\left(\nabla u^{h}-\nabla u\right) \mathrm{d} \Omega}{\int_{\Omega}(\nabla u)^{\mathrm{T}} \nabla u \mathrm{~d} \Omega}} .
\end{gathered}
$$

Figure 11 shows all the convergence curves with respective to the element size of each element. We use $m$ to denote the slope of the convergence curve. From this figure we note that DFEM achieves a convergence rate in the displacement norm $(2<m=2.63<3)$ and for the energy norm $(1<m=1.69<2)$, both of which are between the expected rates for linear and quadratic Lagrange finite elements. And the precision of DFEM is improved than $\operatorname{FEM}(\mathrm{T} 3)$ for $h \sim 10^{-2}$. 


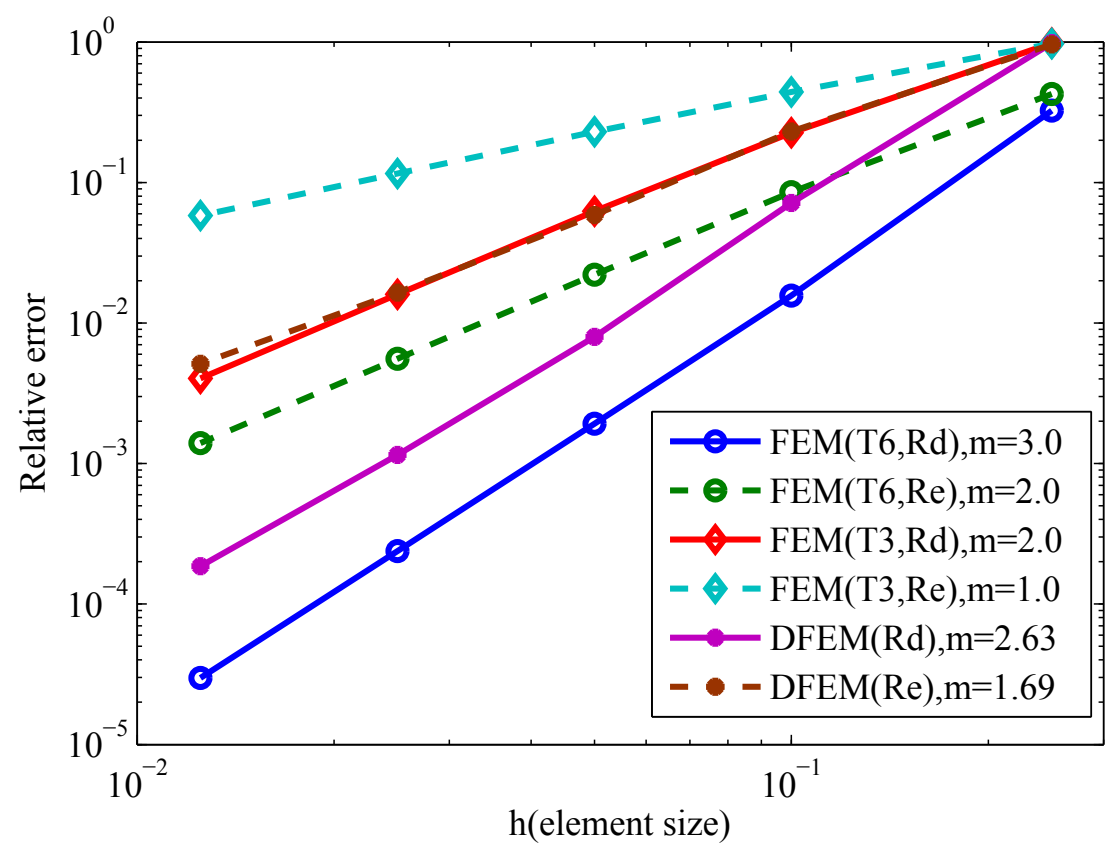

Figure 11: results of the higher-order convergence test

\subsection{Infinite plate with a hole}

Figure 12 presents the upper right quadrant of an infinite plate with a center hole subjected to remote tensile loads. The geometrical parameters are $L=5$ and $a=1$. The analytical solutions for stress and displacement fields are given as [51]:

$$
\begin{gathered}
\sigma_{x x}(r, \theta)=1-\frac{a^{2}}{r^{2}}\left(\frac{3}{2} \cos 2 \theta+\cos 4 \theta\right)+\frac{3 a^{4}}{2 r^{4}} \cos 4 \theta \\
\sigma_{y y}(r, \theta)=-\frac{a^{2}}{r^{2}}\left(\frac{1}{2} \cos 2 \theta-\cos 4 \theta\right)-\frac{3 a^{4}}{2 r^{4}} \cos 4 \theta \\
\tau_{x y}(r, \theta)=1-\frac{a^{2}}{r^{2}}\left(\frac{1}{2} \sin 2 \theta+\sin 4 \theta\right)+\frac{3 a^{4}}{2 r^{4}} \sin 4 \theta \\
u_{r}(r, \theta)=\frac{a}{8 \mu}\left[\frac{r}{a}(\kappa+1) \cos \theta+\frac{2 a}{r}((1+\kappa) \cos \theta+\cos 3 \theta)-\frac{2 a^{3}}{r^{3}} \cos 3 \theta\right], \\
u_{\theta}(r, \theta)=\frac{a}{8 \mu}\left[\frac{r}{a}(\kappa-1) \sin \theta+\frac{2 a}{r}((1-\kappa) \sin \theta+\sin 3 \theta)-\frac{2 a^{3}}{r^{3}} \sin 3 \theta\right],
\end{gathered}
$$

where $(r, \theta)$ are the polar coordinates. The exact traction is imposed on the top and right boundary of the model. The number of nodes used in the four models are 121, 441, 1681 and 6561.

In this example, the numerical results obtained using DFEM, FEM(T3) and FEM(T6) are compared for the same mesh. The relative error in the displacement and energy norm for this example are plotted in Figures 13 and 14, respectively. It can be observed DFEM exceeds the linear optimal convergence rate slightly in the displacement norm, but the error shows an level close to one order of magnitude less than that of T3 elements. In the energy norm, the DFEM converges $34 \%$ faster than the T3 but $31 \%$ slower than the T6, thus providing an intermediate behavior between the two triangular elements. 


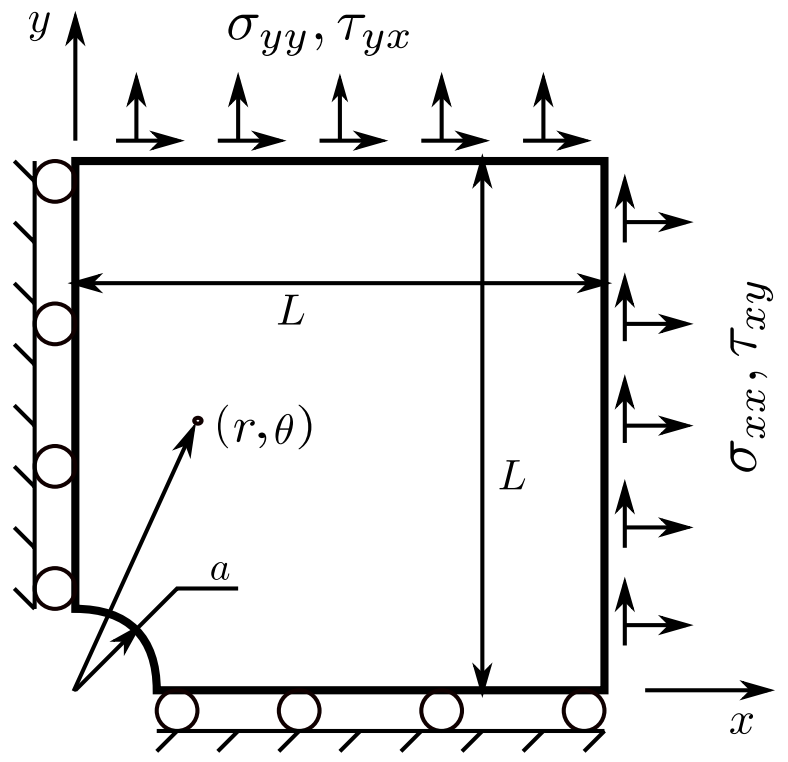

(a)

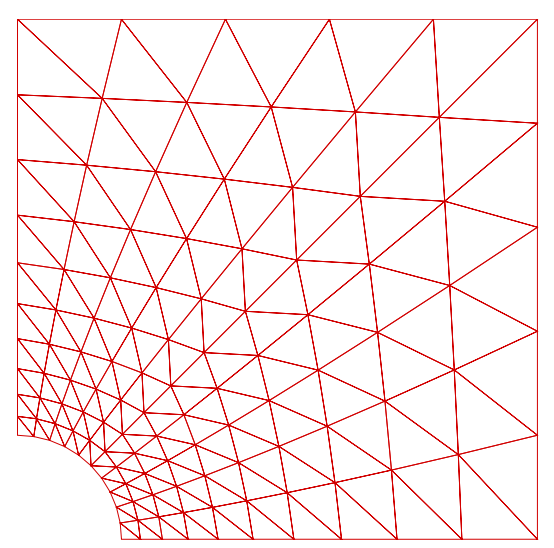

(b)

Figure 12: (a) 1/4 model of the infinite plate with a center hole; (b) The typical mesh division

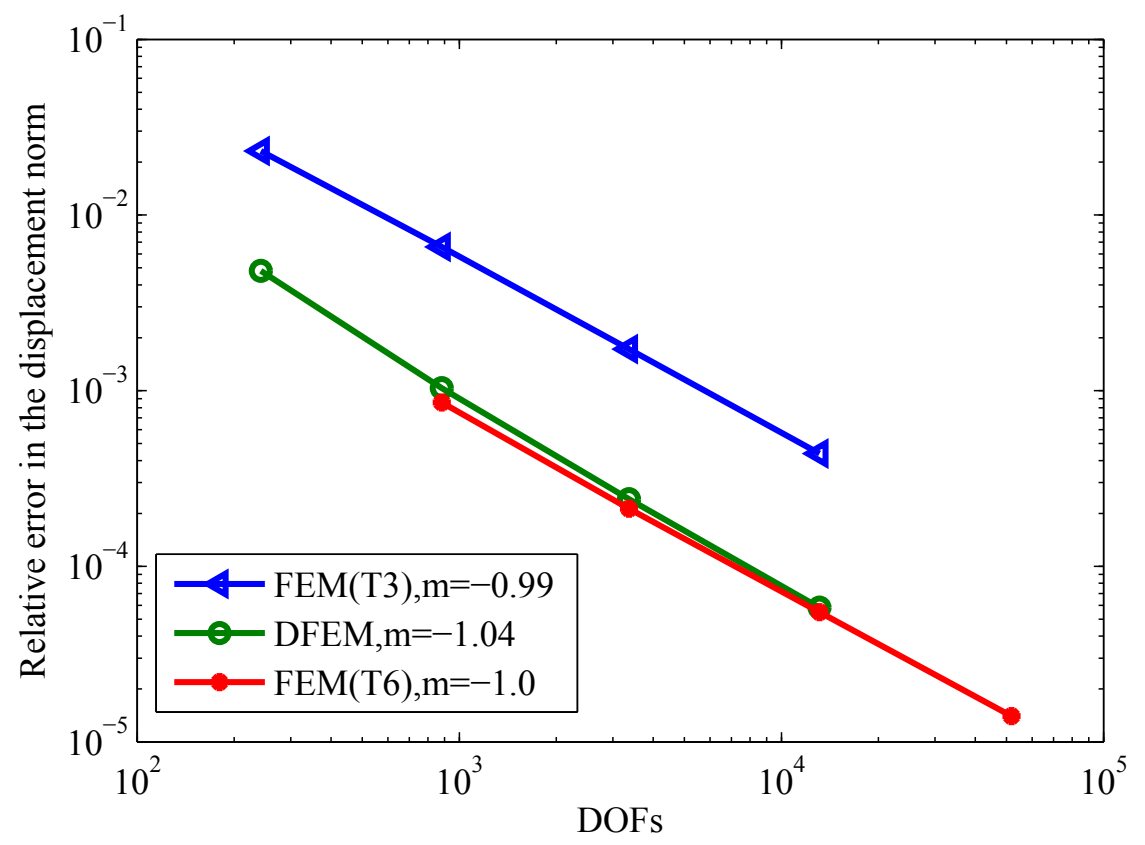

Figure 13: Relative error in the displacement norm for an infinite plate with a hole 


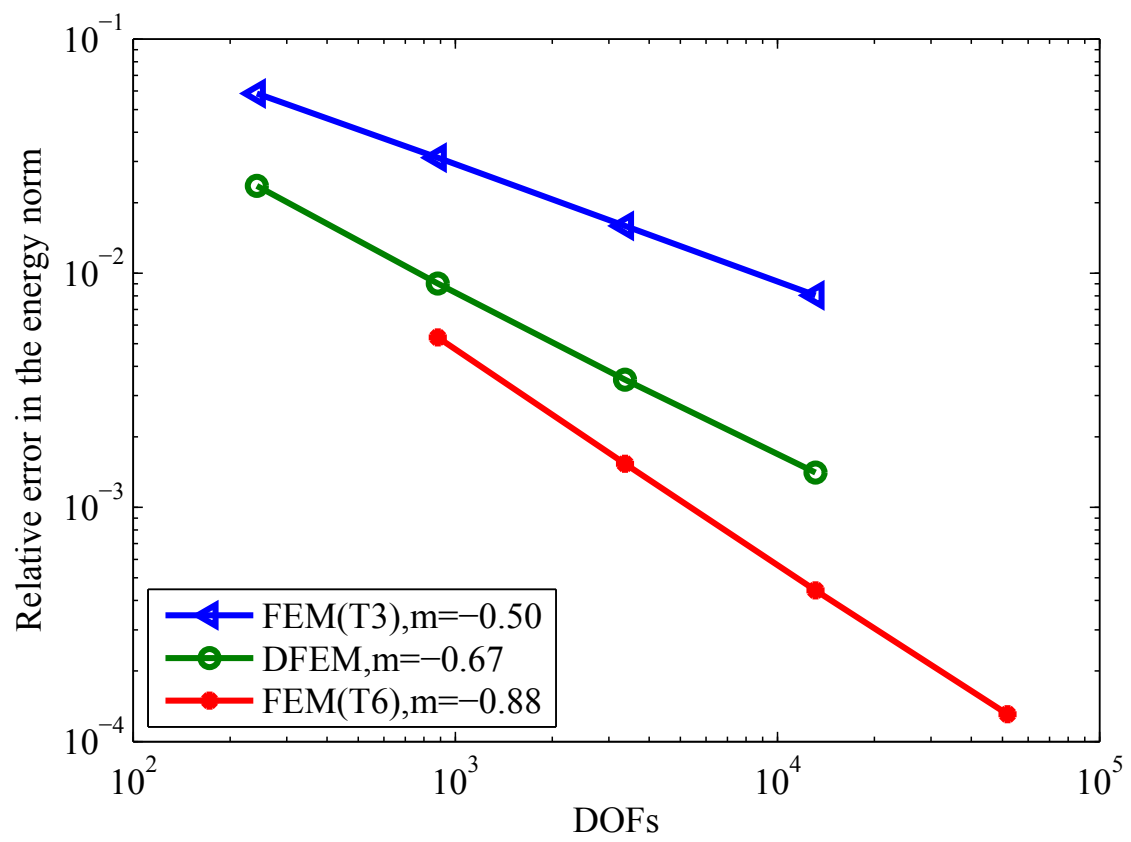

Figure 14: Relative error in the energy norm for an infinite plate with a hole

\subsection{Timoshenko Beam}

Figure 15 illustrates a continuum model of a cantilever beam. In this example, plane stress conditions are assumed. The geometric parameters are taken as $L=48$ and $W=12$. The analytical displacement and stress fields are given in [51] as:

$$
\begin{gathered}
u_{x}(x, y)=\frac{P y}{6 E I}\left[(6 L-3 x) x+(2+\nu)\left(y^{2}-\frac{W^{2}}{4}\right)\right] \\
u_{y}(x, y)=-\frac{P}{6 E I}\left[3 \nu y^{2}(L-x)+(4+5 \nu) \frac{W^{2} x}{4}+(3 L-x) x^{2}\right] \\
\sigma_{x x}(x, y)=\frac{P(L-x) y}{I} \\
\sigma_{y y}(x, y)=0 \\
\tau_{x y}(x, y)=-\frac{P}{2 I}\left(\frac{W^{2}}{4}-y^{2}\right) .
\end{gathered}
$$

where $P=1000$. and $I=W^{3} / 12$. The exact displacement is applied to the left boundary and the exact traction is applied to the right boundary.

Structured meshes are used in this example to ensure regular node location and to enable easier comparison among the T3, T6, Q4 and DFEM (Figure 16). Four mesh sizes, $3 \times 10,6 \times 20$, $12 \times 40$ and $24 \times 80$, are used. It can be observed that, the DFEM solution demonstrates better accuracy and is super-convergent in the displacement and energy norm by more than $50 \%$ compared to Q4 and T3, but is inferior to T6 in both accuracy and convergence rate. Note that $\mathrm{T} 6$ achieves much better accuracy for the Timoshenko beam due to the analytical solution is of complete quadratic order. 


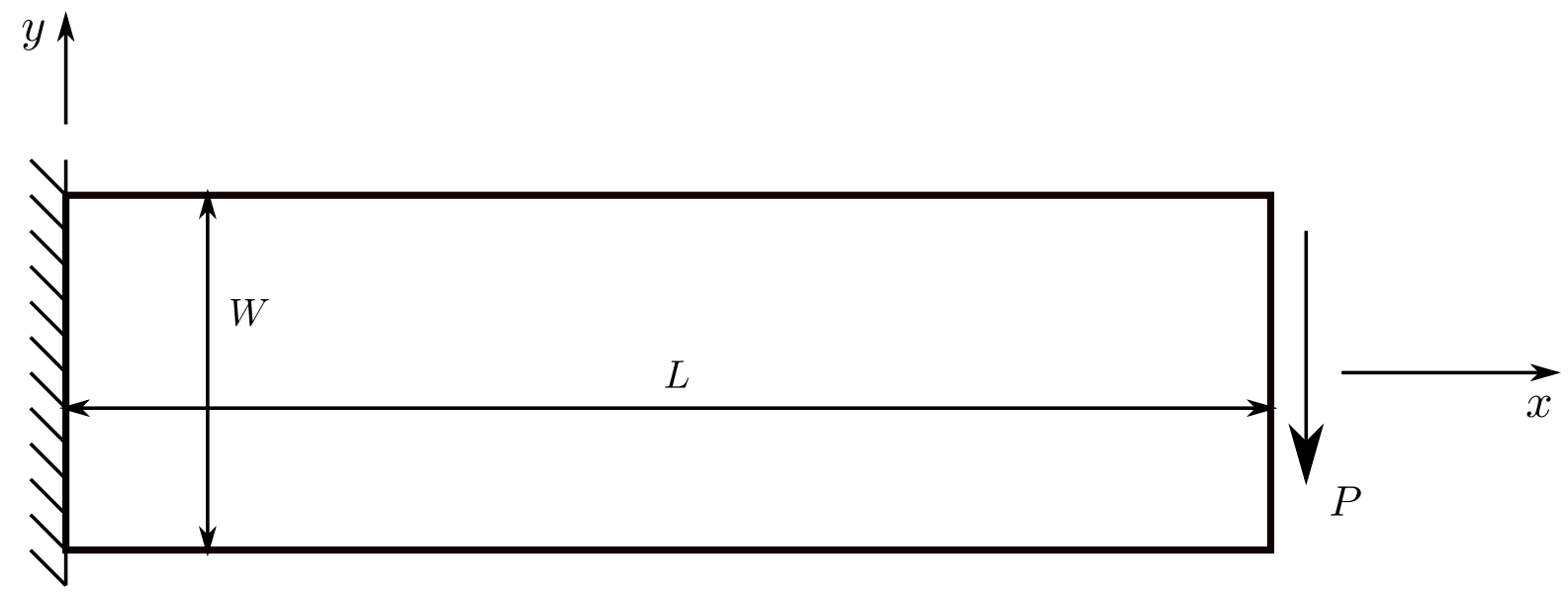

Figure 15: Physical model of cantilever beam
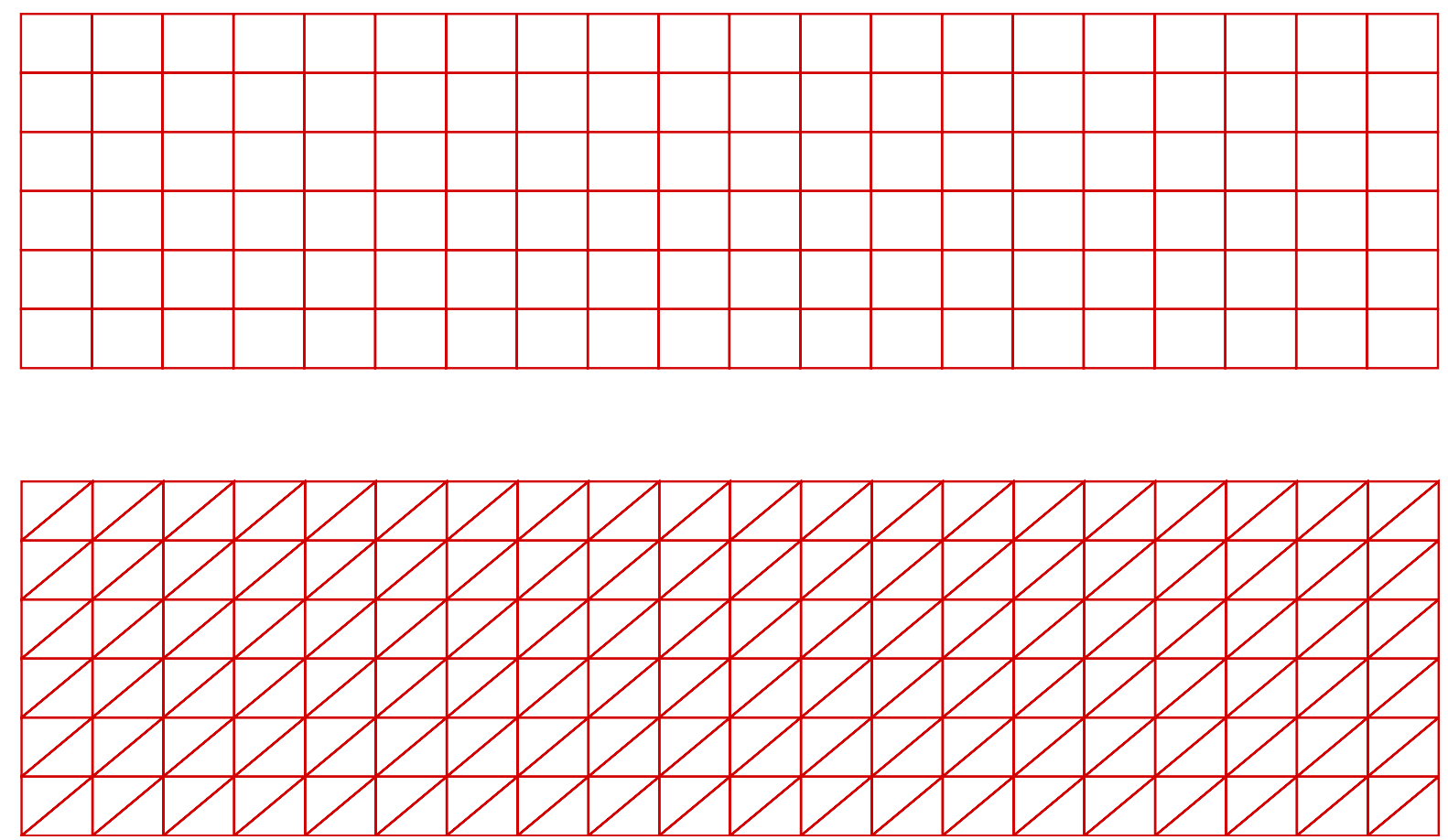

Figure 16: Mesh discretization using regular quadrilateral and triangular elements 


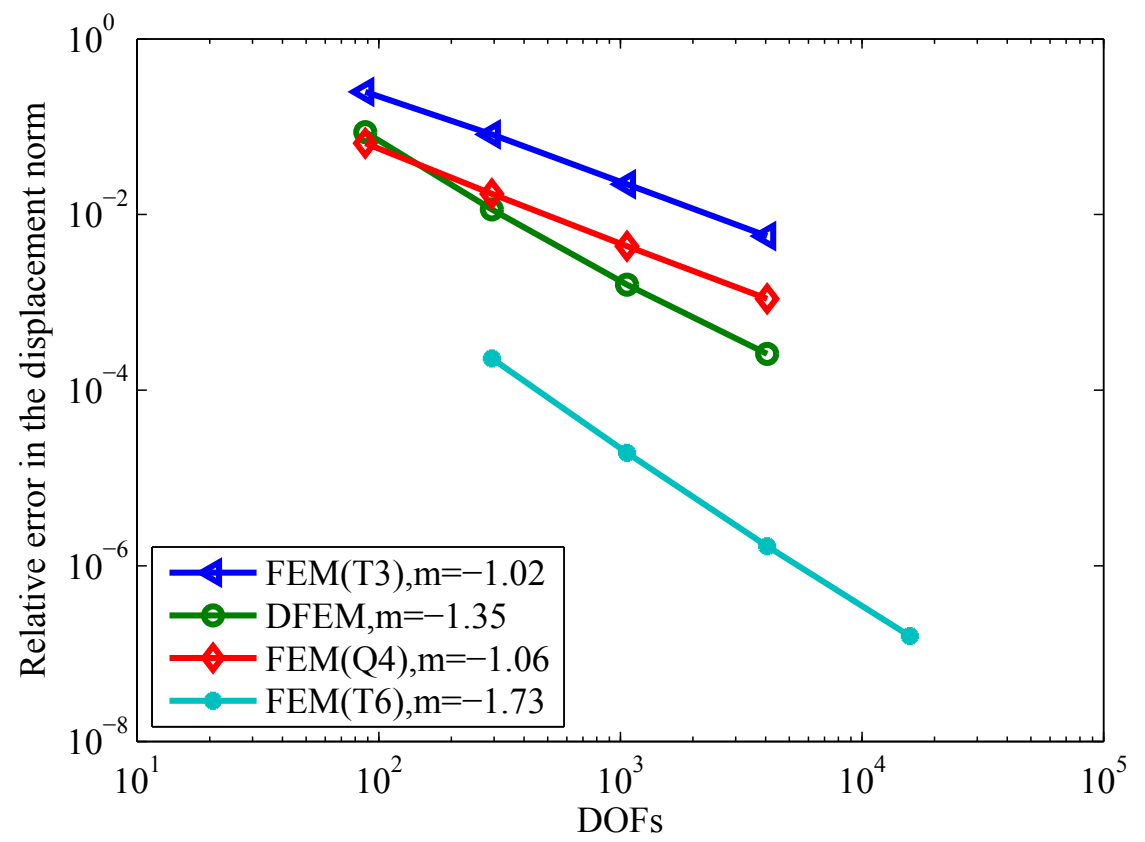

(a)

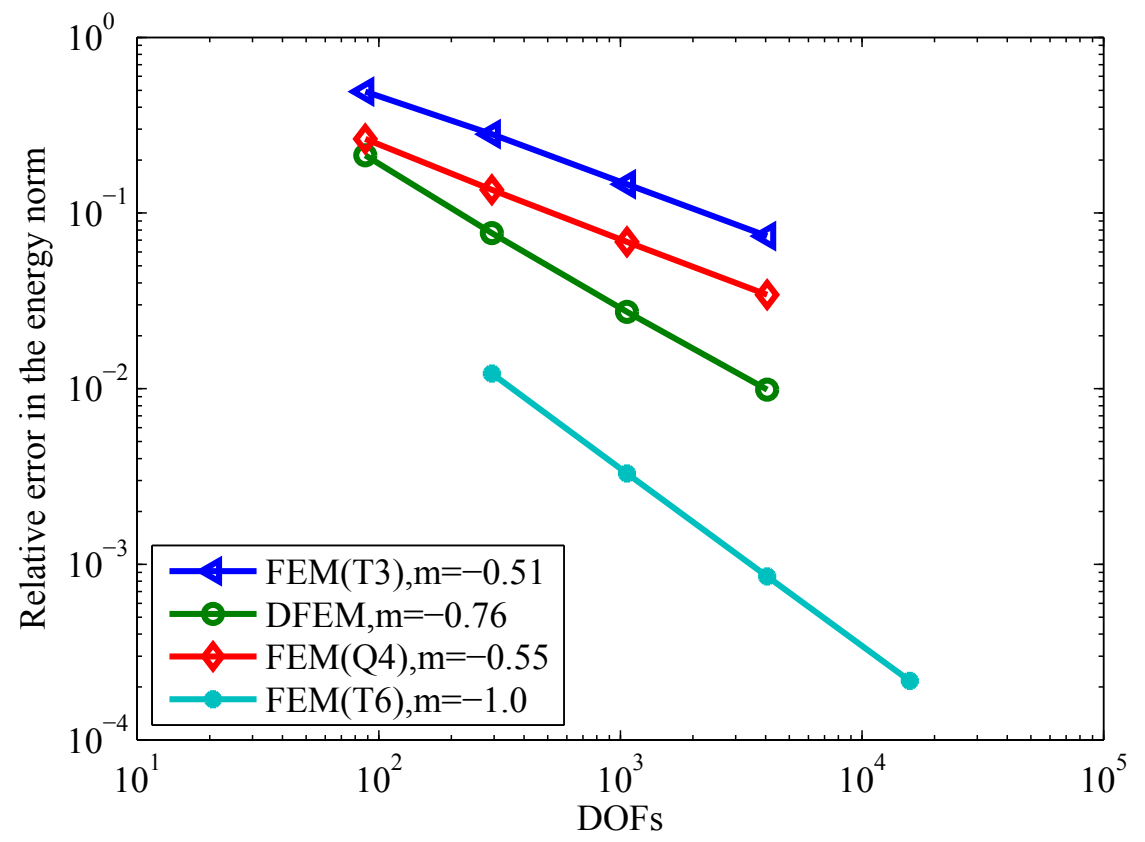

(b)

Figure 17: Relative error in displacement and energy norm of Timoshenko beam 


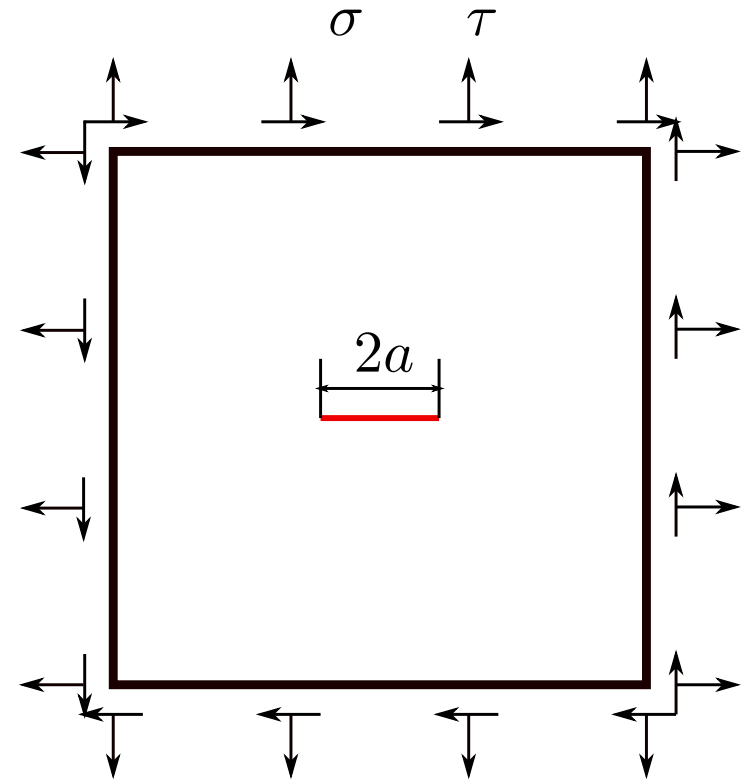

(a)

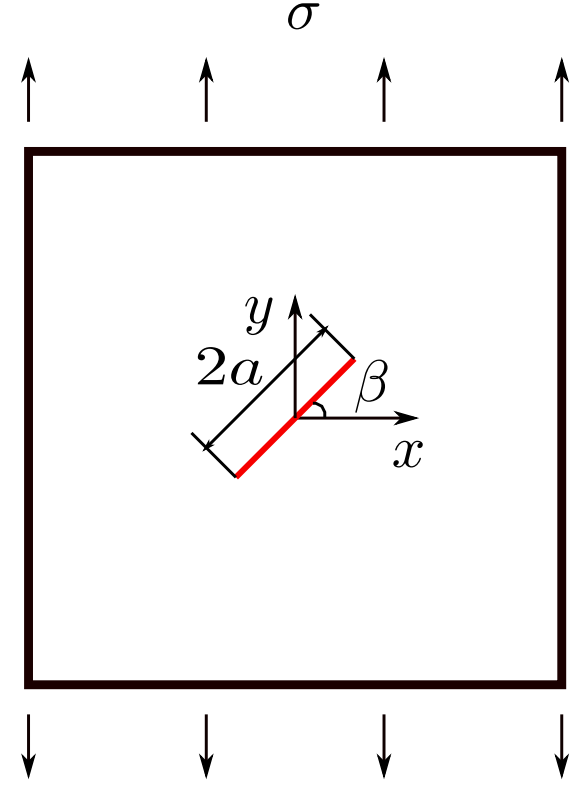

(b)

Figure 18: (a)Griffith crack; (b) inclined crack

\subsection{Griffith crack}

A Griffith crack problem is shown in Figure 18(a). An infinite plate with a crack segment $(\mathrm{a}=1$. subjected to remote tensile loads is considered here. A square domain $(10 \times 10)$ is selected in the vicinity of the crack tip. The analytical displacement and stress fields are given by [52]:

$$
\begin{gathered}
\sigma_{x x}(r, \theta)=\frac{K_{I}}{\sqrt{2 \pi r}} \cos \frac{\theta}{2}\left(1-\sin \frac{\theta}{2} \sin \frac{3 \theta}{2}\right) \\
-\frac{K_{I I}}{\sqrt{2 \pi r}} \sin \frac{\theta}{2}\left(2+\cos \frac{\theta}{2} \cos \frac{3 \theta}{2}\right), \\
\sigma_{y y}(r, \theta)=\frac{K_{I}}{\sqrt{2 \pi r}} \cos \frac{\theta}{2}\left(1+\sin \frac{\theta}{2} \sin \frac{3 \theta}{2}\right)+\frac{K_{I I}}{\sqrt{2 \pi r}} \sin \frac{\theta}{2} \cos \frac{\theta}{2} \cos \frac{3 \theta}{2}, \\
\tau_{x y}(r, \theta)=\frac{K_{I}}{\sqrt{2 \pi r}} \sin \frac{\theta}{2} \cos \frac{\theta}{2} \cos \frac{3 \theta}{2}+\frac{K_{I I}}{\sqrt{2 \pi r}} \cos \frac{\theta}{2}\left(1-\sin \frac{\theta}{2} \sin \frac{3 \theta}{2}\right), \\
u_{x}(r, \theta)=\frac{K_{I}}{2 \mu} \sqrt{\frac{r}{2 \pi}} \cos \frac{\theta}{2}\left(\kappa-1+2 \sin ^{2} \frac{\theta}{2}\right) \\
+\frac{(1+\nu) K_{I I}}{E} \sqrt{\frac{r}{2 \pi}} \sin \frac{\theta}{2}\left(\kappa+1+2 \cos ^{2} \frac{\theta}{2}\right), \\
u_{y}(r, \theta)= \\
\frac{K_{I}}{2 \mu} \sqrt{\frac{r}{2 \pi}} \sin \frac{\theta}{2}\left(\kappa+1-2 \cos ^{2} \frac{\theta}{2}\right) \\
+\frac{(1+\nu) K_{I I}}{E} \sqrt{\frac{r}{2 \pi}} \cos \frac{\theta}{2}\left(1-\kappa+2 \sin ^{2} \frac{\theta}{2}\right),
\end{gathered}
$$

where $K_{I}$ and $K_{I I}$ are the stress intensity factors (SIFs) for mode-I and mode-II, respectively. $(r, \theta)$ are the polar coordinates used to define the crack geometry. 


\subsubsection{Convergence study}

The Griffith crack problem is first used to investigate the enrichment effects of DFEM. The convergence rate in XDFEM is studied in three ways: explicit crack representation (where the crack is explicitly meshed), Heaviside enrichment only and full Heaviside and asymptotic enrichment. These results are plotted in Figure 19. From Figure 19, it can be concluded that the DFEM yields better accuracy and slightly improves the convergence rate compared to FEM for all the cases considered. It also transpires from the results that the full enrichment of DFEM produces better accuracy than modelling the crack explicitly. Note that we make no correction for blending issues in partially enriched elements [18].

1 million DOF problems in both mode-I and mode-II were simulated to assess the convergence rate of the method (see Figures 20 and 21). The relative errors in the SIFs are also shown in the plots. We study the case where only tip enrichment is used, which is known [26][27] to lead to the same convergence rate as the standard Lagrange-based FEM, and which is confirmed here also for XDFEM, as expected.

We also observe that XDFEM is, as XFEM, able to reproduce the discontinuity across the crack faces. When geometrical enrichment with an enrichment radius of $1 / 5$ of the crack length is used (Figure 22), optimality is recovered and the XDFEM solution is also more accurate than the XFEM solution in terms of displacement, energy and SIFs. Figure 23 illustrates the number of iterations required for the Conjugate Gradient (CG) solver to converge, which can be regarded as an indication to the condition number of the stiffness matrix. It is observed that XDFEM performs slightly worse than XFEM in terms of the condition number. As expected, when a fixed area enrichment is used, the deterioration of the condition number is accentuated compared to the case when only the element containing the tip is enriched. These conclusions are in agreement with the investigation reported in [26][28].

\subsubsection{Accuracy study}

Though it was already established from the convergence curves that over the whole computational domain, the XDFEM is generally slightly more accurate than XFEM for a given number of DOFs, it is necessary in practice to investigate whether XDFEM improves the precision also locally in the vicinity of the crack tip. The strain component $\epsilon_{y y}$ is plotted along the line perpendicular to the crack in front of the tip (the line $x=0$ ) in a $31 \times 31$ structured mesh in Figure 24. It can be noted from Figure 24, that the XDFEM result is much closer to the analytical solution than that of XFEM. Especially, in the vicinity of the crack tip, the XDFEM performs better due to the inclusion of nodal gradients in the approximation.

The mesh distortion effect is also investigated in this example. A structured mesh and typical distorted mesh are shown in Figure 25. The results are listed in Table 1. The precision of XDFEM in distorted mesh appears to be superior to that of the XFEM, although this superiority is mild.

\begin{tabular}{|c|c|c|c|c|}
\hline & \multicolumn{2}{|c|}{ Strutured mesh } & \multicolumn{2}{c|}{ distorted mesh } \\
\hline DOFs & XFEM & XDFEM & XFEM & XDFEM \\
\hline 334 & 0.2272 & 0.1832 & 0.2313 & 0.1882 \\
\hline 4726 & 0.1112 & 0.08672 & 0.1132 & 0.08863 \\
\hline 7834 & 0.09769 & 0.07600 & 0.1016 & 0.08261 \\
\hline 17134 & 0.08006 & 0.06212 & 0.08223 & 0.06215 \\
\hline
\end{tabular}

Table 1: Relative error in the energy norm for regular structured meshes and distorted meshes 


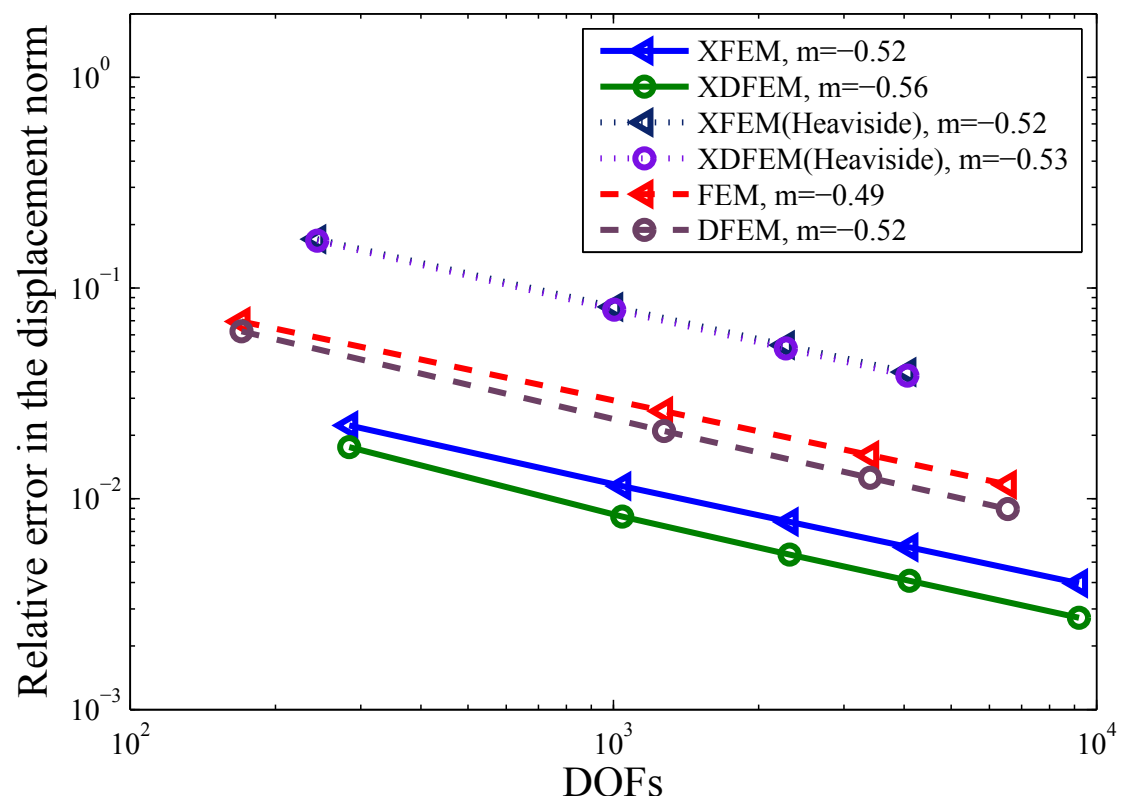

(a)

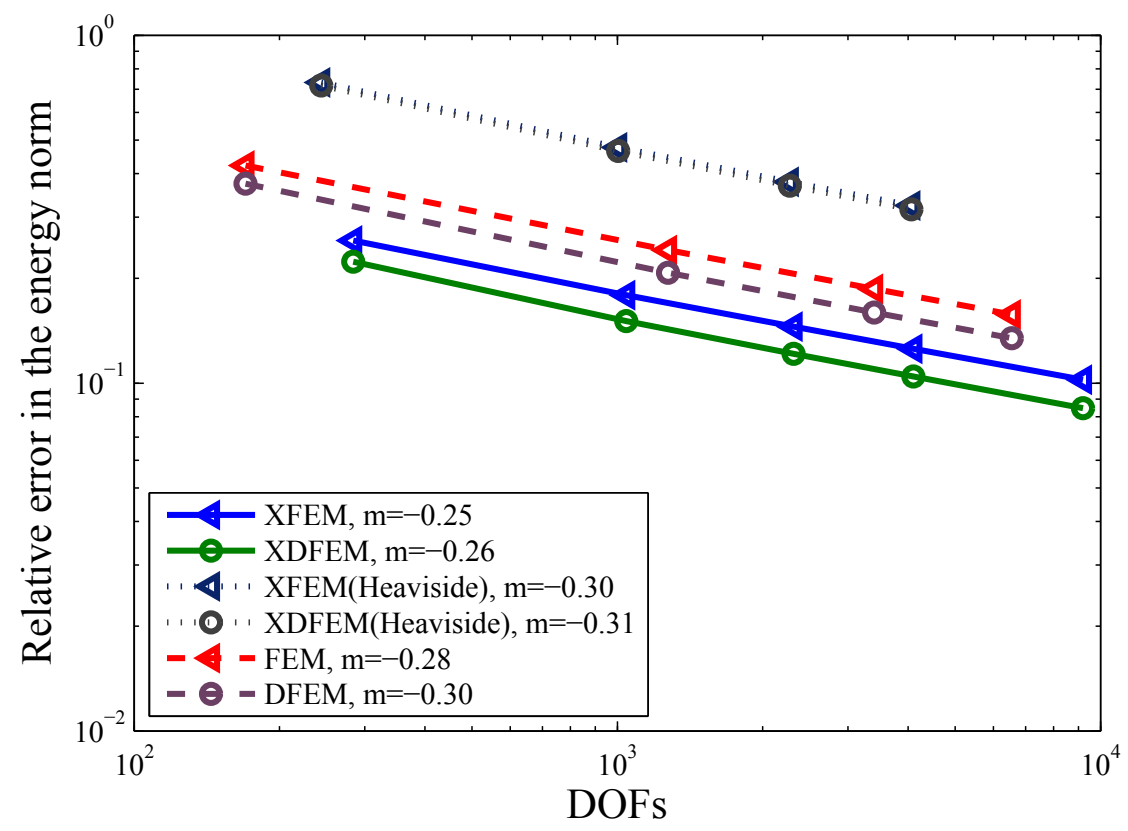

(b)

Figure 19: Relative error in the displacement and energy norm of in Griffith crack for explicit crack representation(dashed lines), Heaviside enrichment only (dotted lines) and topological enrichment (solid lines) 


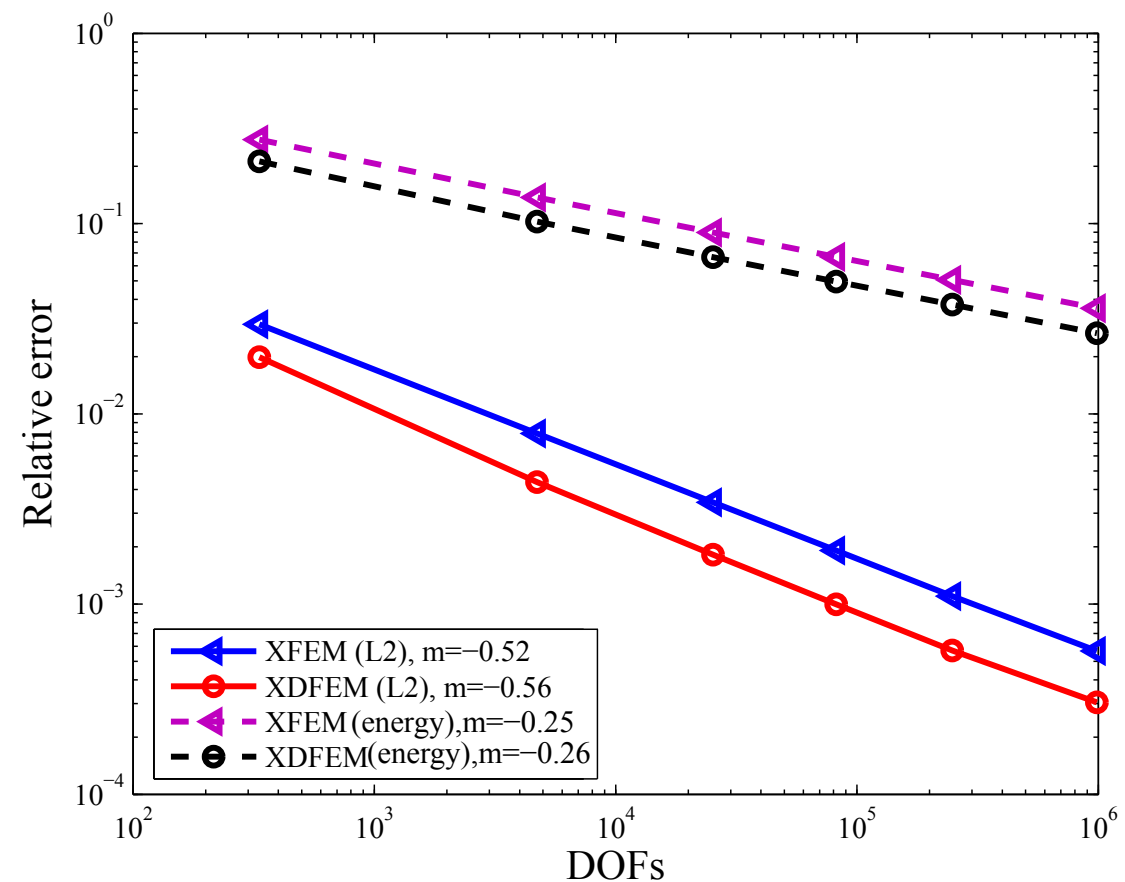

(a)

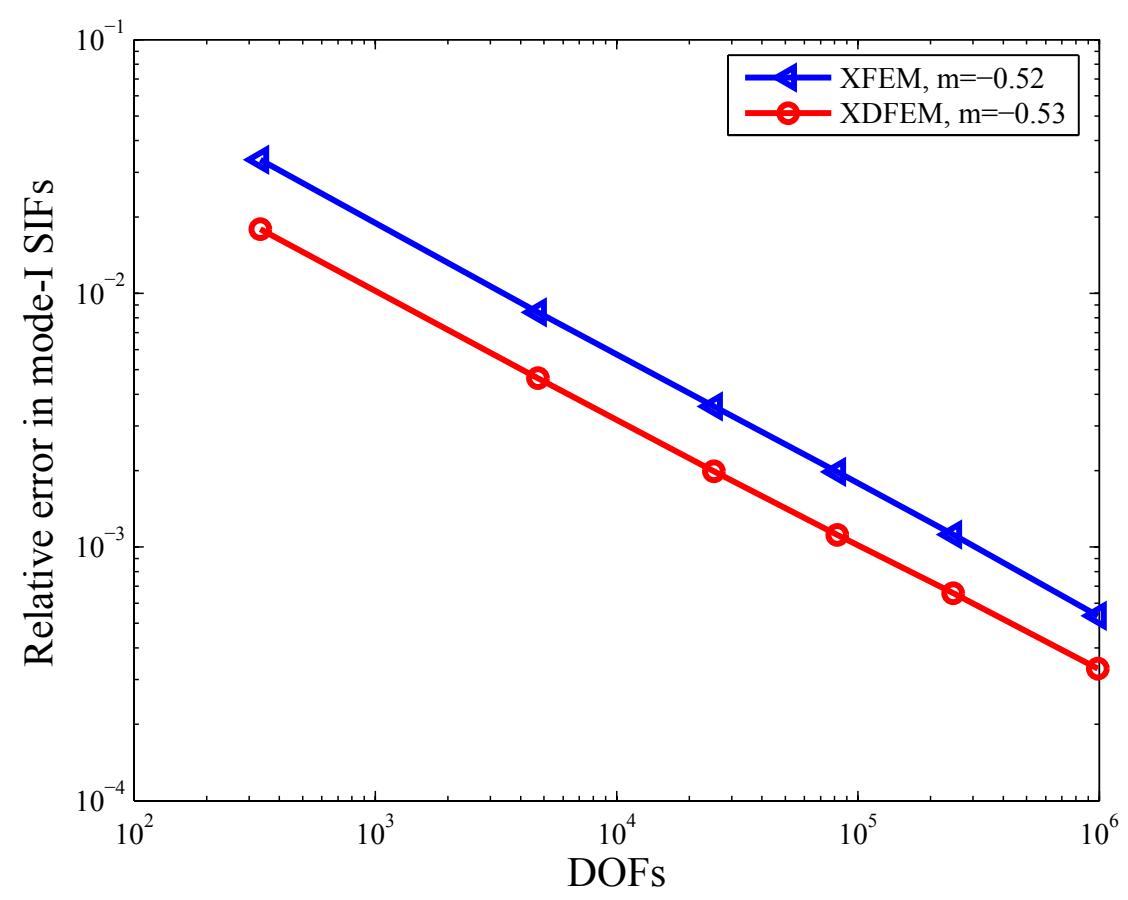

(b)

Figure 20: Convergence results of Griffith crack (mode-I) for topological enrichment: (a) the error in the displacement $L_{2}$ and energy norm; (b) the error in SIFs 


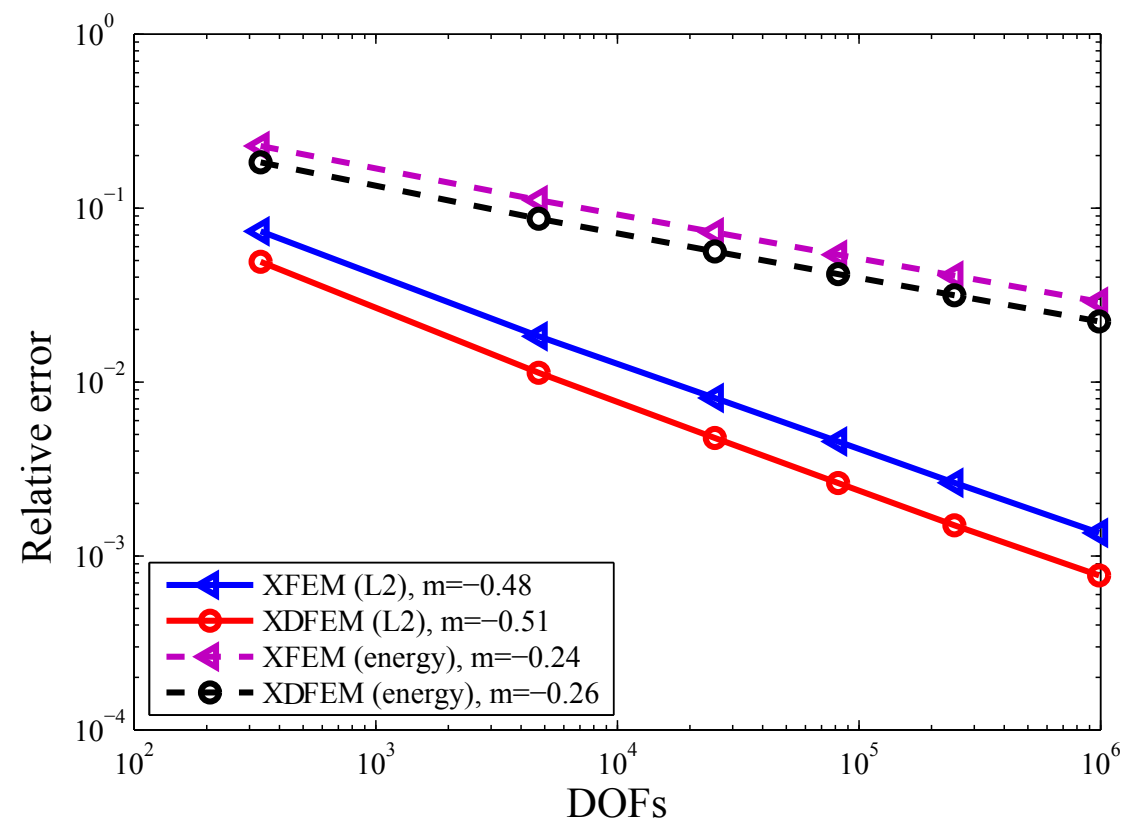

(a)

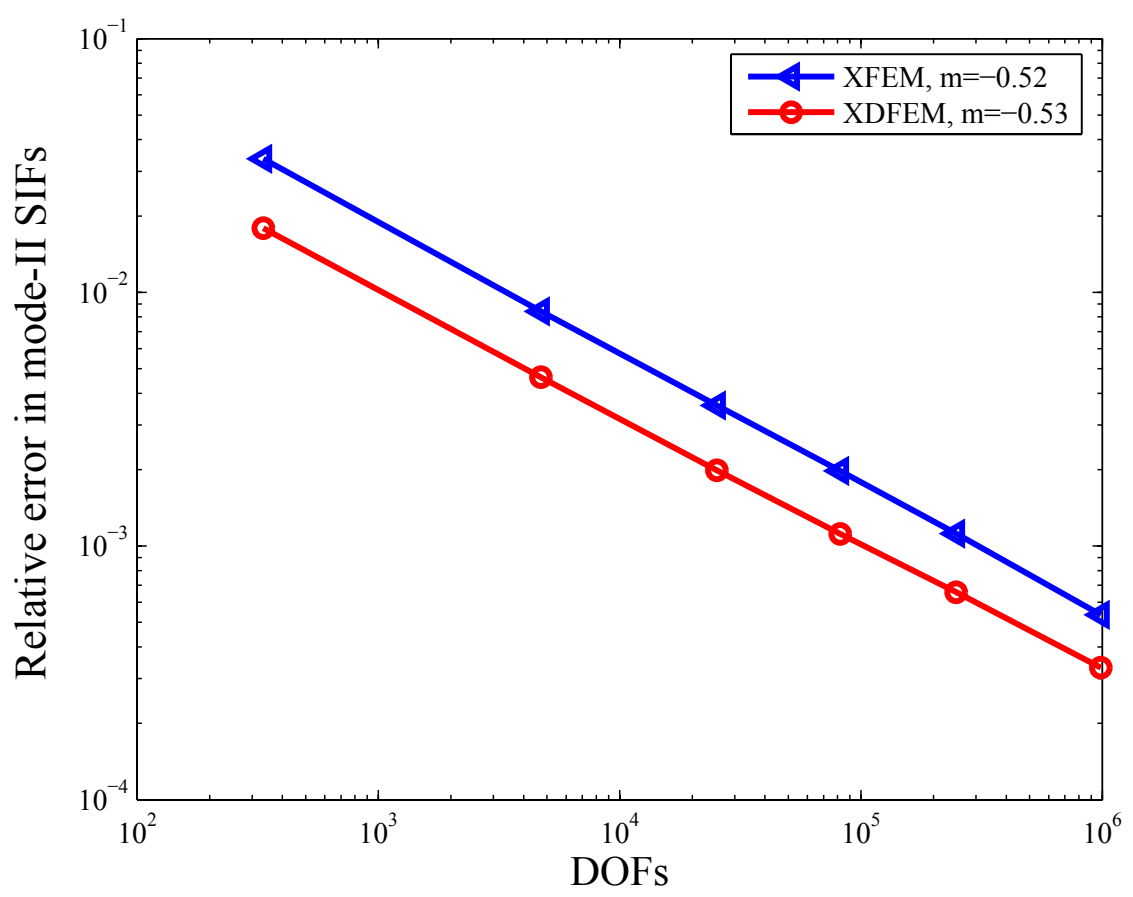

(b)

Figure 21: Convergence results of Griffith crack (mode-II) for topological enrichment: (a) the error in the displacement $L_{2}$ and energy norm; (b) the error in SIFs 


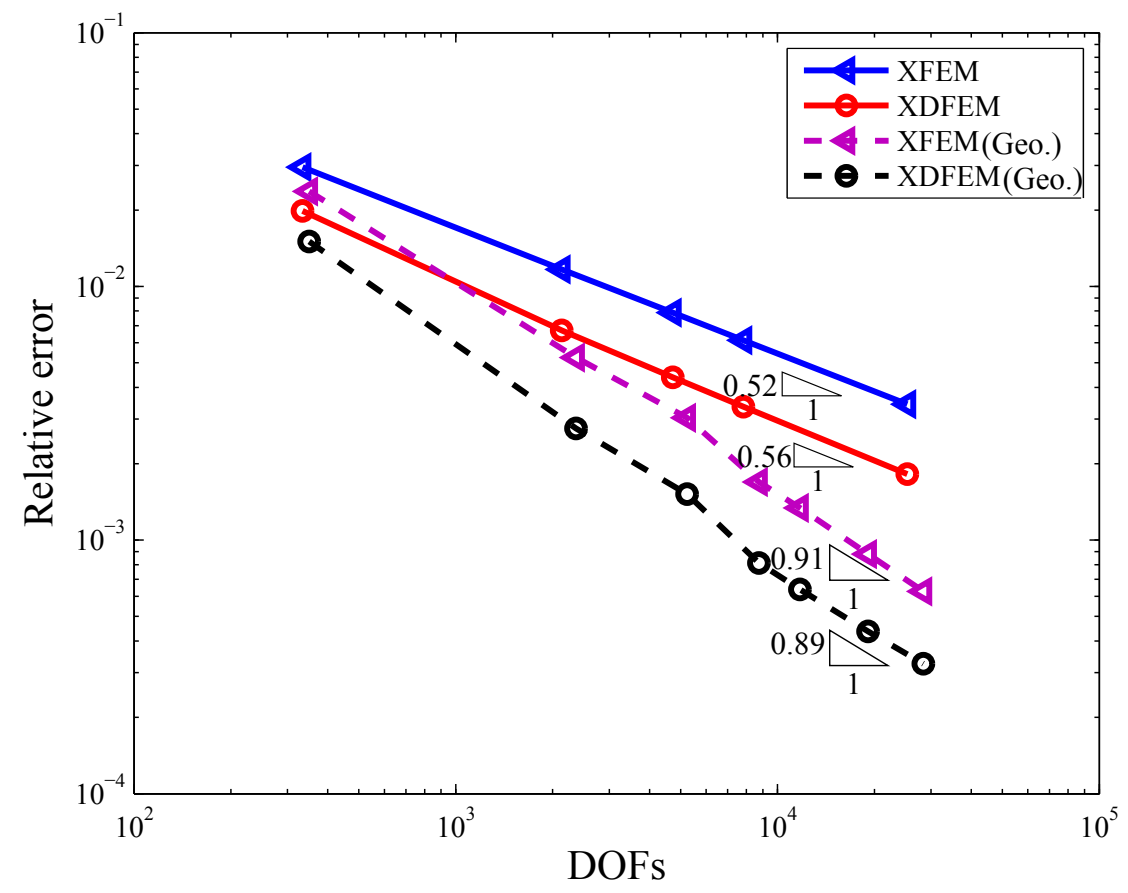

(a)

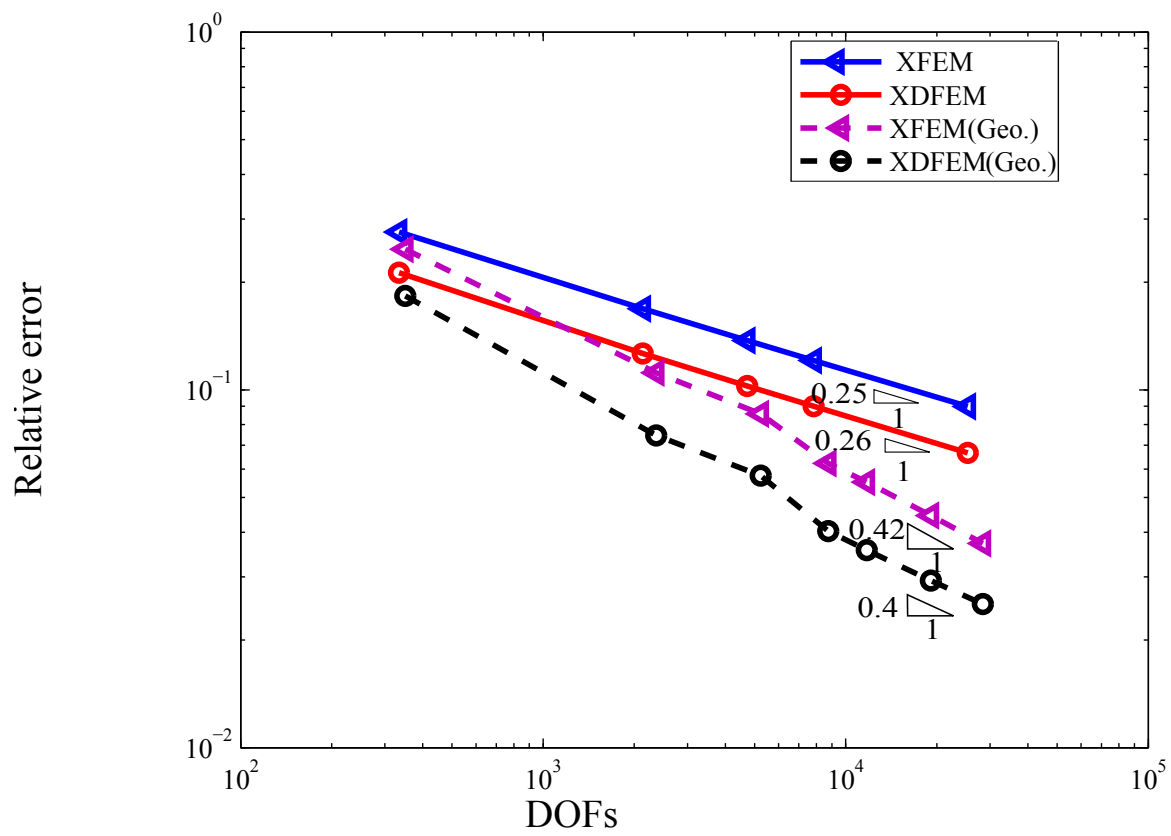

(b)

Figure 22: Convergence results of topological enrichment (solid lines) and geometrical enrichment (Geo., dashed lines) for mode-I: (a) the error in the displacement $L_{2}$ norm; (b) the error in the energy norm 


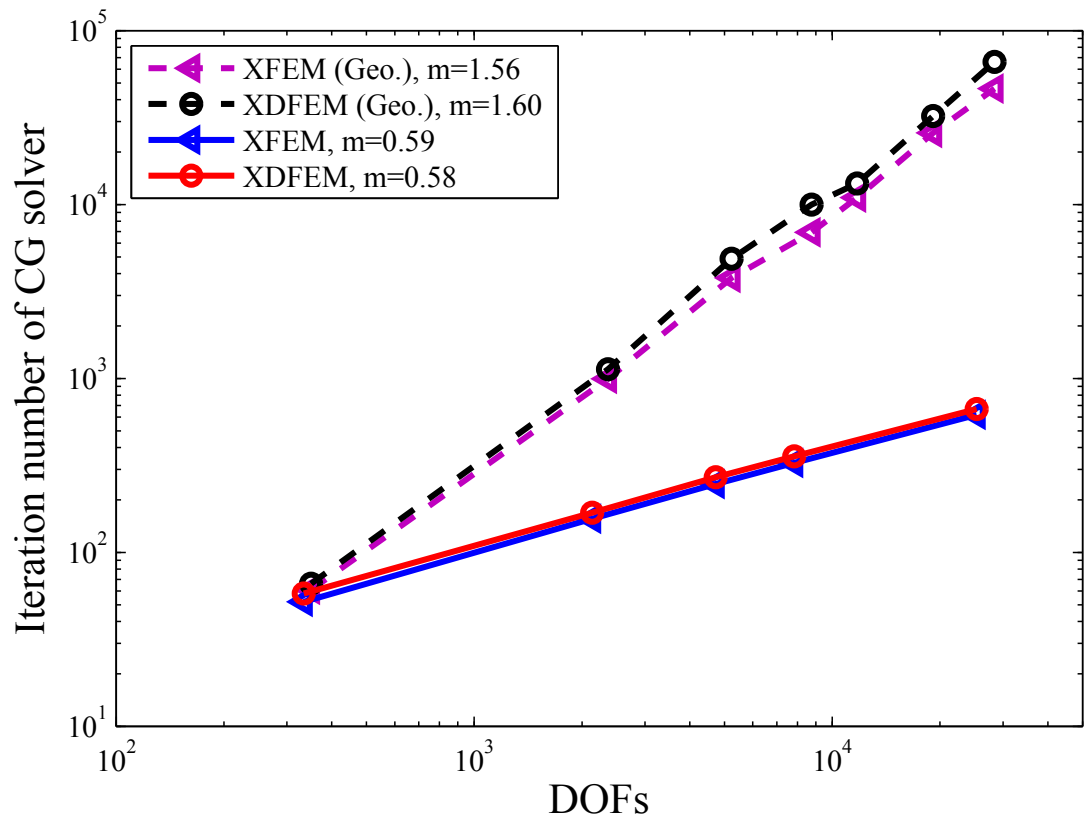

Figure 23: Iteration number of CG solver; solid lines for topological enrichment, dashed lines for geometrical enrichment

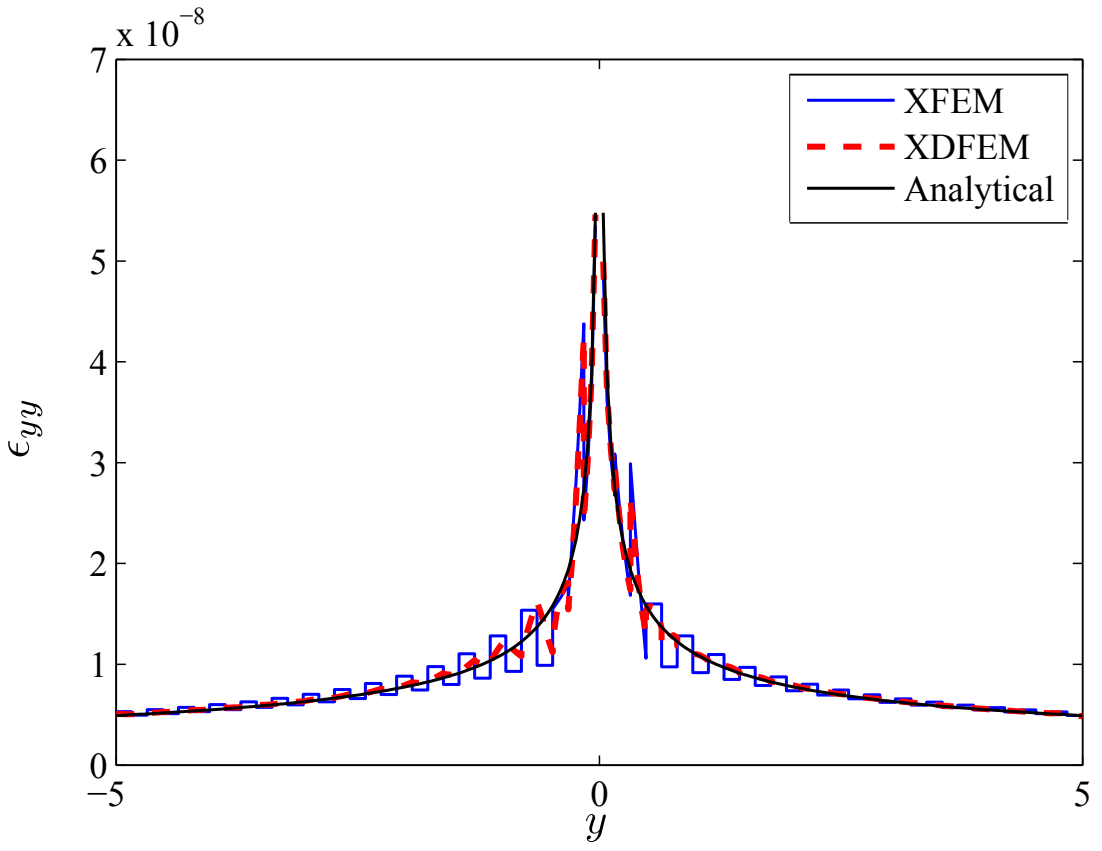

Figure 24: The comparison of the strain component along line $x=0$ 


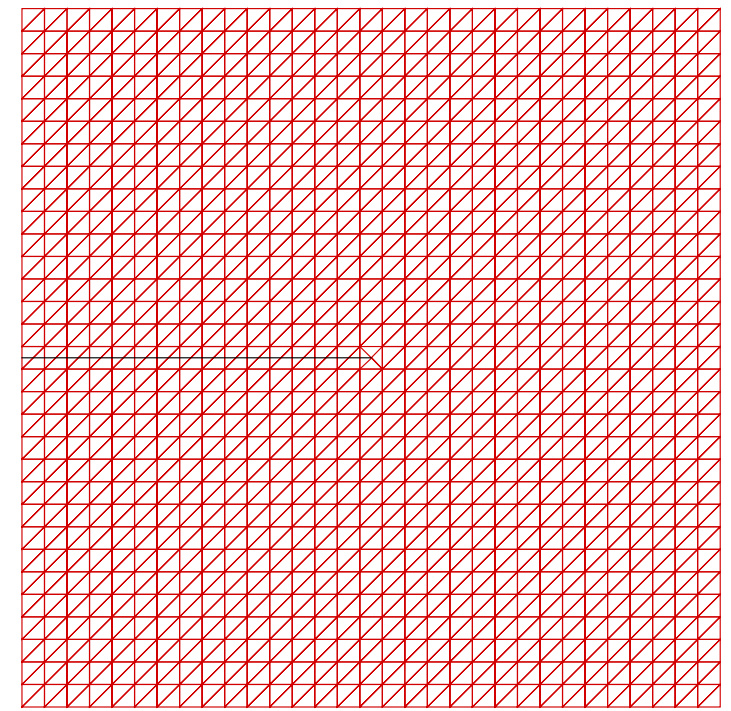

(a)

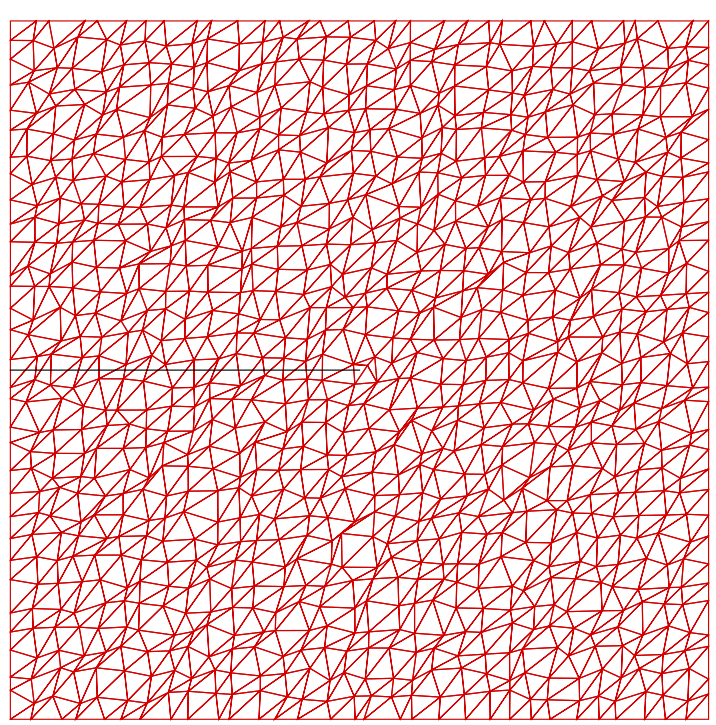

(b)

Figure 25: Mesh design to check the mesh distortion effect: (a)structured mesh; (b) distorted mesh

\subsubsection{Computational efficiency}

It should be highlighted that the support domain of DFEM element is much bigger than that of FEM element due to the introduction of the nodal gradient into the approximation (see Figure 1, Figure 5). This directly results in increased bandwidth of the stiffness matrix in DFEM. Consequently, the computational time per DOFs is expected to be larger for DFEM than for the FEM. Figure 26 and 27(a) show the comparison of the time cost in assembling the stiffness matrix, solving the linear equations and the total time of the two processes. It can be seen that with the model size increasing, XDFEM requires less time to obtain the same precision. For the solution process, XFEM produces an error 1.4 times higher $\left(\frac{X F E M 15.48}{X D F E M 11}=1.4\right)$ than the XDFEM at the same computational time of 0.06 seconds. The total time comparison shows that after $t_{0}=0.6$ seconds, XDFEM is more efficient computationally than XFEM in terms of the energy error. It can be observed from Figure 27(b) that XDFEM is always superior to XFEM in the same DOFs. The main cause of the increased cost associated with XDFEM is the increased bandwidth. This can be alleviated by using an 'element-by-element' approach.

\subsection{Inclined center crack}

An inclined crack problem is investigated in this section. The model is presented in Figure 18(b). The infinite plate is subjected to remote tensile load in $y$ direction and the inclination angle $\beta$ is measured in the counter-clockwised direction from the $x$ direction. The half crack length is $a=1$. A square domain field $(10 \times 10)$ encircling the crack tip is selected and the exact displacement is applied on the boundary, as in the previous example. The analytical SIFs are given as

$$
\begin{gathered}
K_{I}=\sigma \sqrt{\pi a} \cos ^{2} \beta, \\
K_{I I}=\sigma \sqrt{\pi a} \cos \beta \sin \beta .
\end{gathered}
$$

Table 2 shows the relative error of $K_{I}$ and $K_{I I}$ varying with the inclination angle of the crack. It can be observed from Table 2 that both XDFEM and XFEM results agree well with the analytical solution. The precision of the SIFs in the XDFEM are better than that of the 


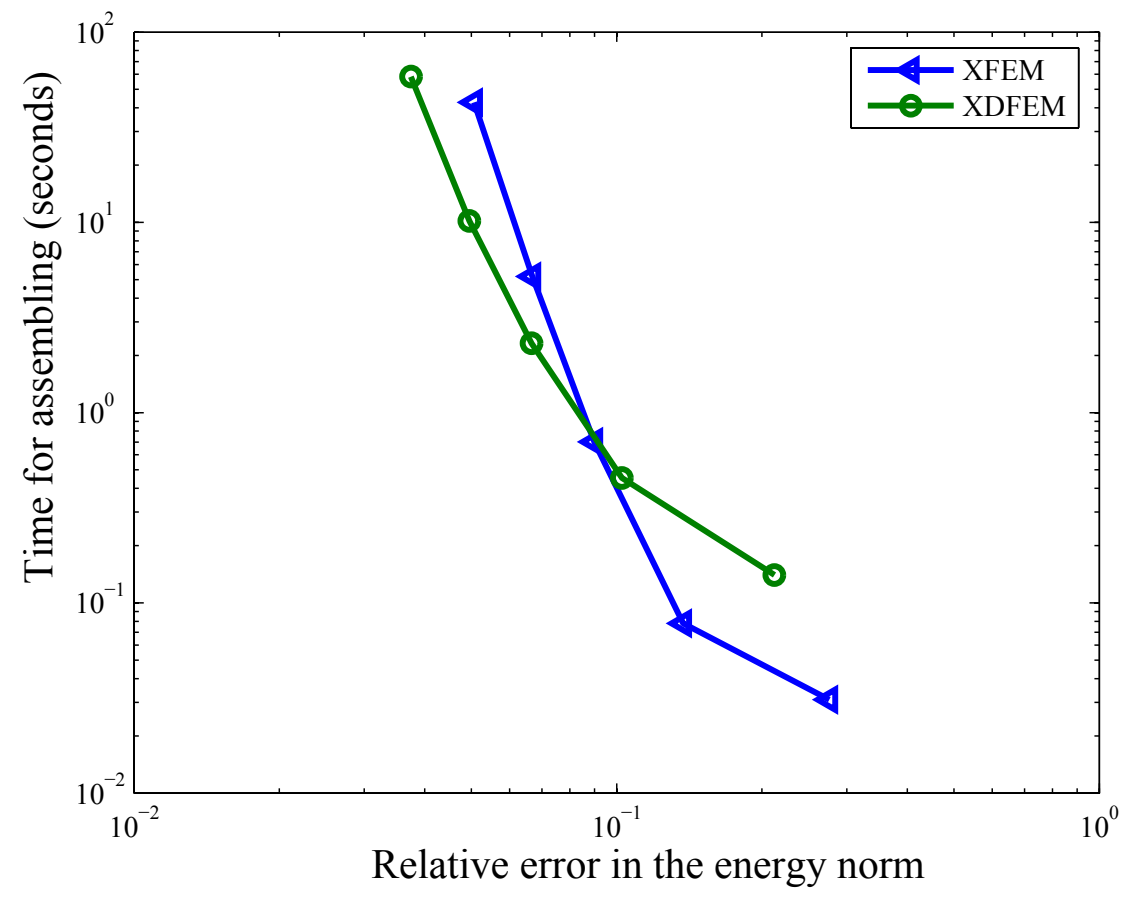

(a)

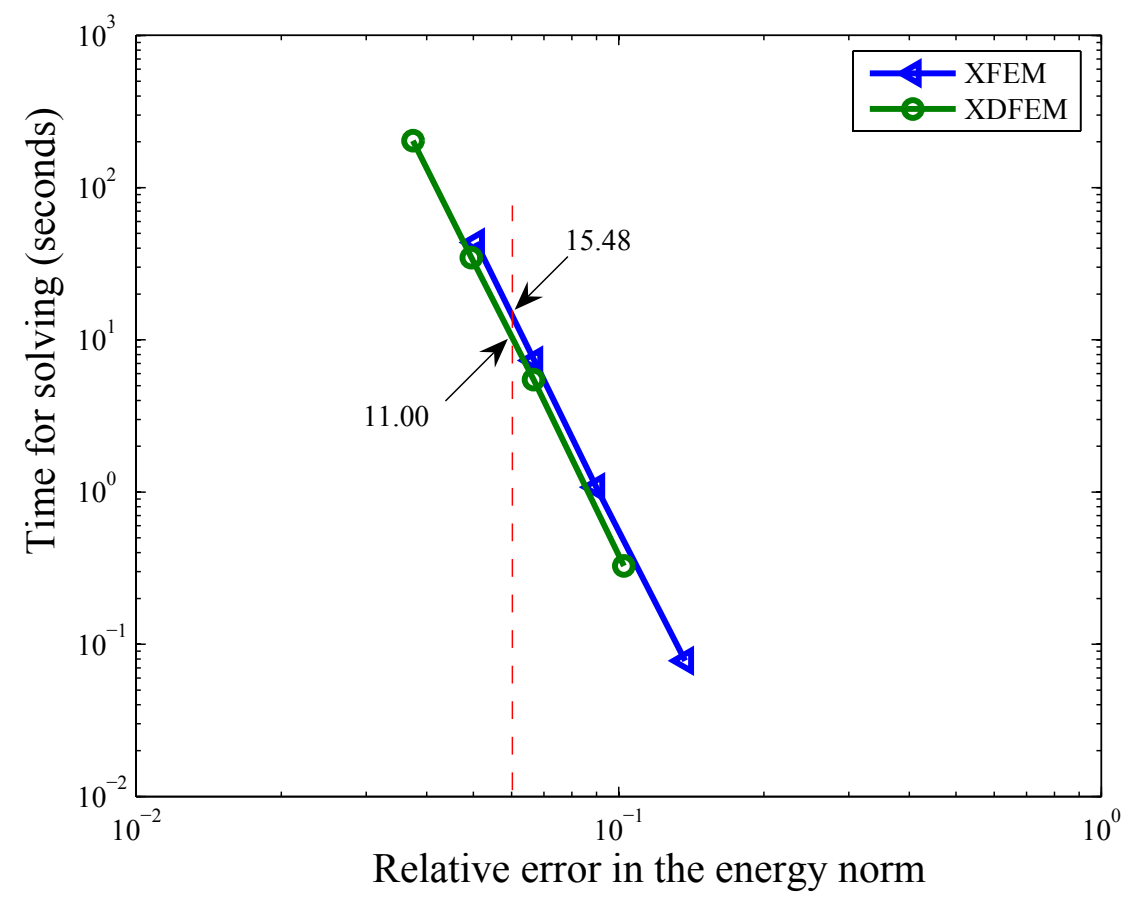

(b)

Figure 26: Comparison of time costs for XFEM and XDFEM in Griffith crack problem 


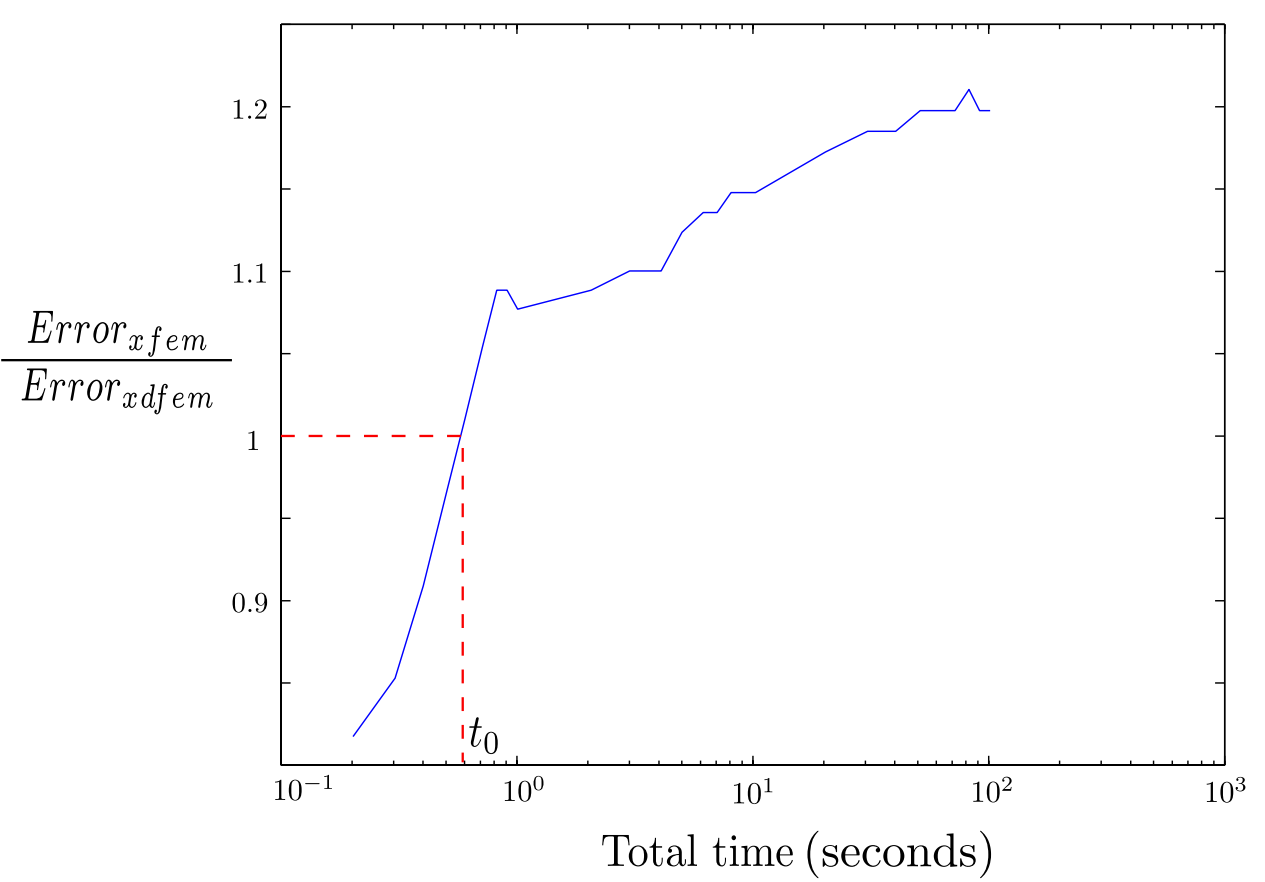

(a)

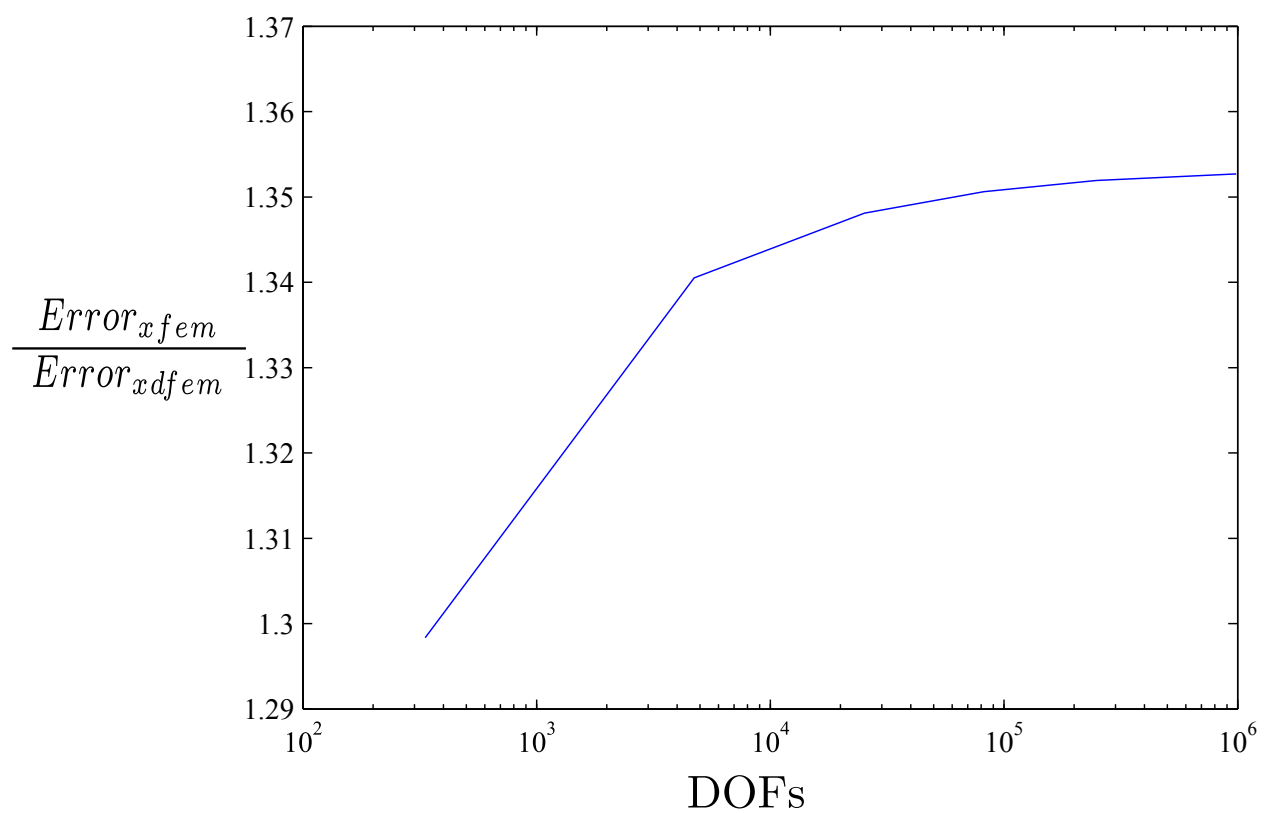

(b)

Figure 27: The comparison of the energy norm error in terms of (a) time; (b) DOFs 


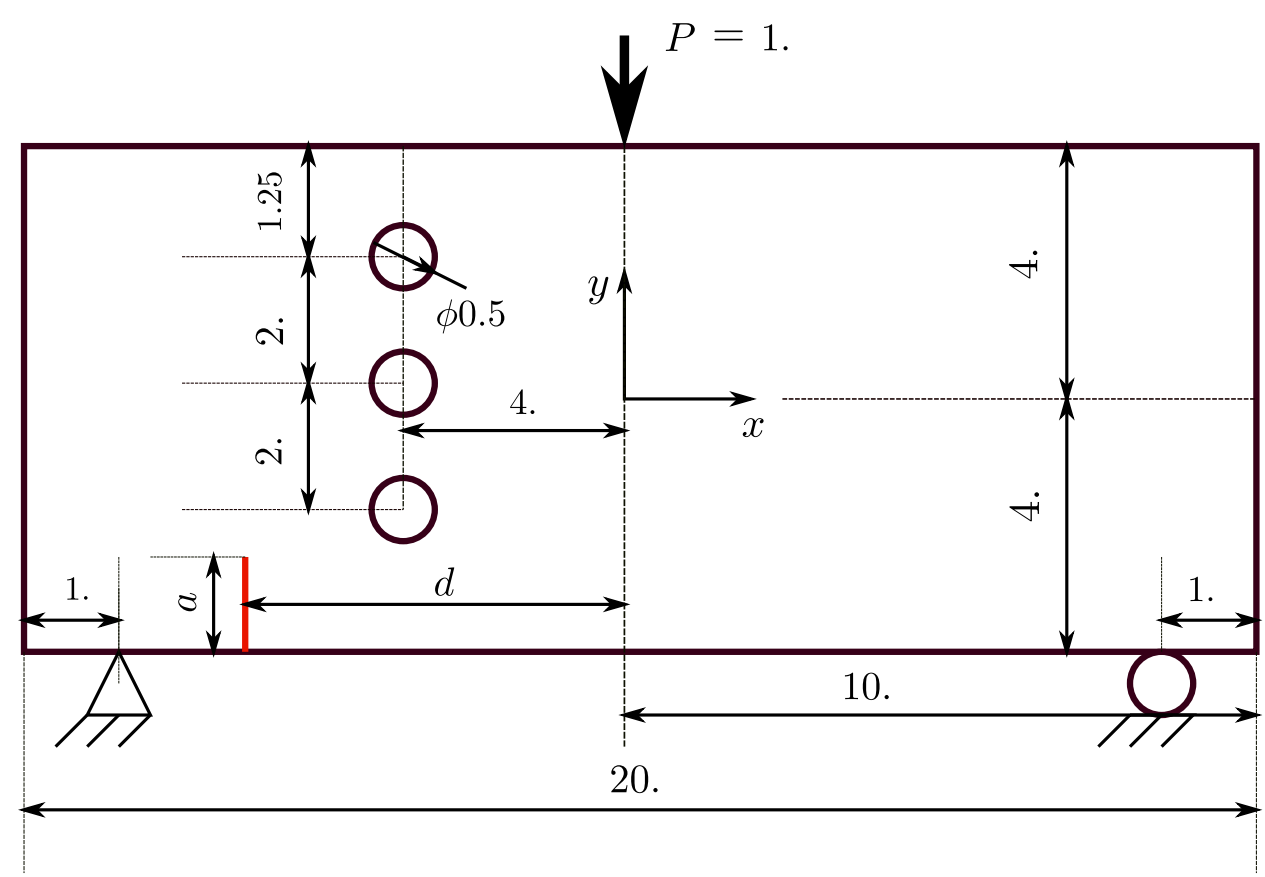

Figure 28: Physical model of three points bending beam with three holes

XFEM. This example demonstrates that XDFEM performs well also for the mixed mode crack problems.

\begin{tabular}{|c|c|c|c|c|}
\hline & \multicolumn{2}{|c|}{$K_{I}(\%)$} & \multicolumn{2}{c|}{$K_{I I}(\%)$} \\
\hline$\beta$ & XFEM & XDFEM & XFEM & XDFEM \\
\hline 0 & 0.58 & 0.29 & 0.03 & 0.10 \\
\hline$\frac{\pi}{12}$ & 0.54 & 0.28 & 0.07 & 0.12 \\
\hline$\frac{\pi}{6}$ & 0.49 & 0.26 & 0.30 & 0.20 \\
\hline$\frac{\pi}{4}$ & 0.43 & 0.23 & 0.36 & 0.21 \\
\hline$\frac{\pi}{3}$ & 0.32 & 0.20 & 0.41 & 0.23 \\
\hline$\frac{5 \pi}{12}$ & $<10^{-3}$ & 0.14 & 0.43 & 0.23 \\
\hline$\frac{\pi}{2}$ & $<10^{-3}$ & $<10^{-3}$ & $<10^{-3}$ & $<10^{-3}$ \\
\hline
\end{tabular}

Table 2: The error in the SIFs for the inclined center crack problem $(47 \times 47$ structured mesh). XDFEM reduces the error by a factor of up to 2.0

\subsection{XDFEM for crack propagation}

A three point bending beam with three holes is simulated in this section to test the versatility of XDFEM in simulating crack propagation. Holes strongly influence crack propagation in structures and the chosen example is a decisive test for computational fracture problems, as the crack path obtained is most sensitive to the accuracy of the crack driving force computation, as well as the chosen propagation increment, as will be seen below. This experiment is designed to explore the effect of holes on the crack trajectories. The geometry and load condition are illustrated in Figure 28. Plexiglas specimens are used for which $E=1000$ and $\nu=0.37$ is used in the simulations. Plane strain condition is assumed. With the variation of the position of the initial crack, different crack trajectories are obtained [53] [54]. A set of test cases, as listed in Table 3, are simulated. The maximum hoop stress criterion and the equivalent domain form 
of the interaction energy integral for SIFs extraction [1] is adopted to compute the orientation of crack propagation. The model is discretized by 27869 nodes and 55604 triangular elements. Figure 29 illustrates the crack evolution of the listed three cases. And the results show that both methods are in good agreement with the experiment. In the numerical tests it is noted that, although the error in the energy norm lower in XDFEM, it can be observed from Figure 29 that, there is very minor difference in the crack path trajectory between XFEM and XDFEM. However the crack paths obtained from both methods show a significant deviation when the crack passes the hole in case 1 and case 3 . We should somehow be aware that the different crack increment will affect the crack path as noticed in [55]. The SIFs for the three crack trajectories are plotted in Figure 30. It can be observed that the SIFs tend to change in a bigger amplitude when the crack approaches the hole in case 1 and case 3. The XFEM and XDFEM SIFs for each case compare well. Figure 31 compares the stress contours of the XFEM and the XDFEM. The XDFEM provides smooth stress fields without any post-processing.

\begin{tabular}{|c|c|c|c|c|}
\hline & $d$ & $a$ & crack increment & number of propagation \\
\hline case 1 & 5 & 1.5 & 0.052 & 67 \\
\hline case 2 & 6 & 1.0 & 0.060 & 69 \\
\hline case 3 & 6 & 2.5 & 0.048 & 97 \\
\hline
\end{tabular}

Table 3: Test cases for the three points bending beam problem
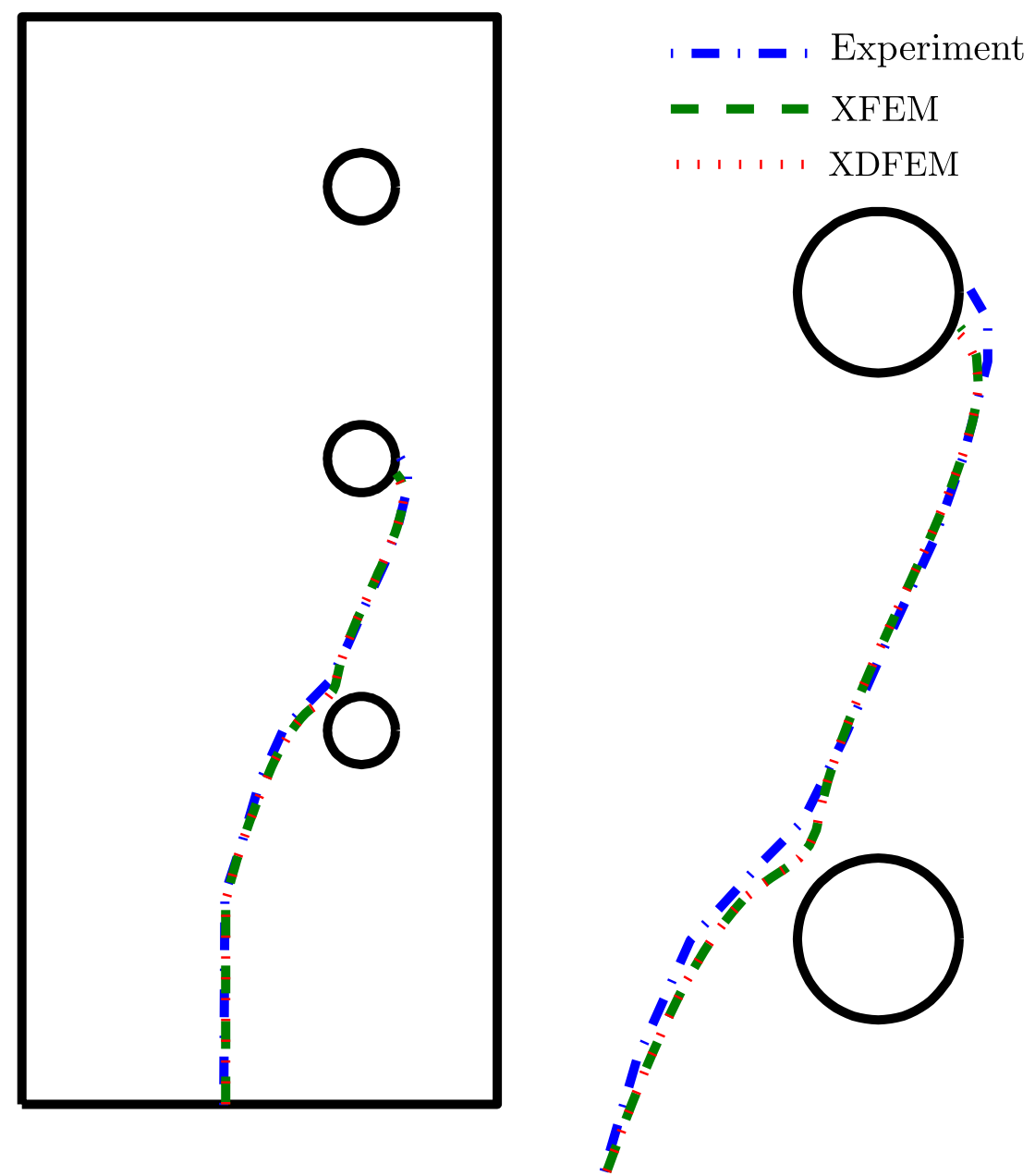

(a) 


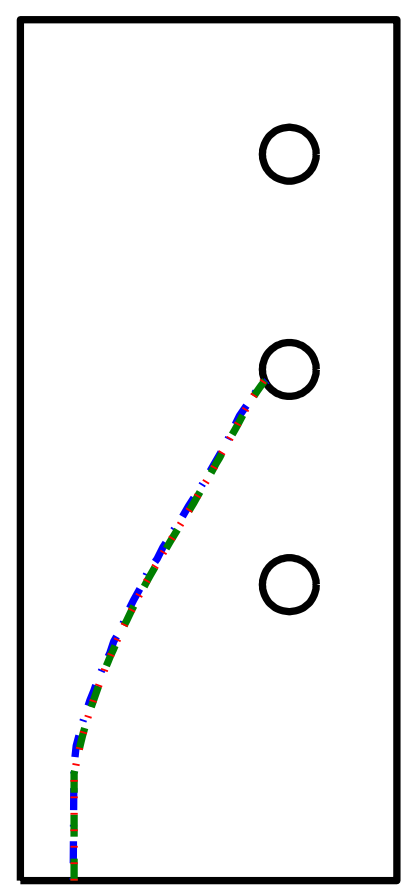

- - Experiment

- - - XFEM

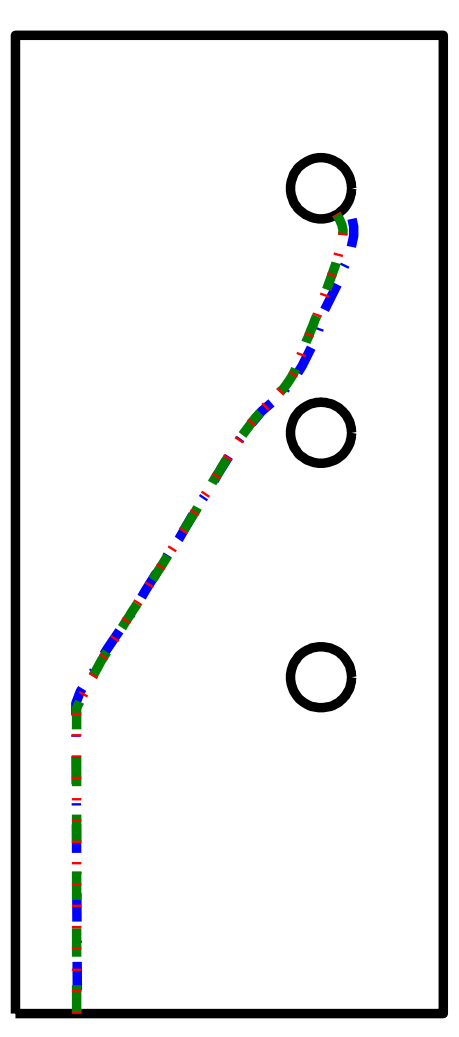

(b)

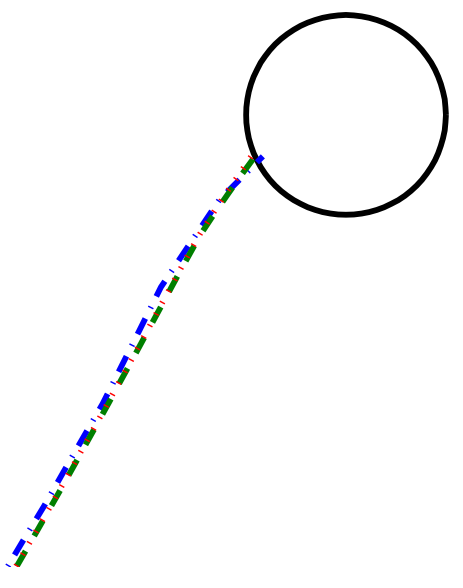

- - - Experiment

- - XFEM

! י 1 , י 1 , XDFEM

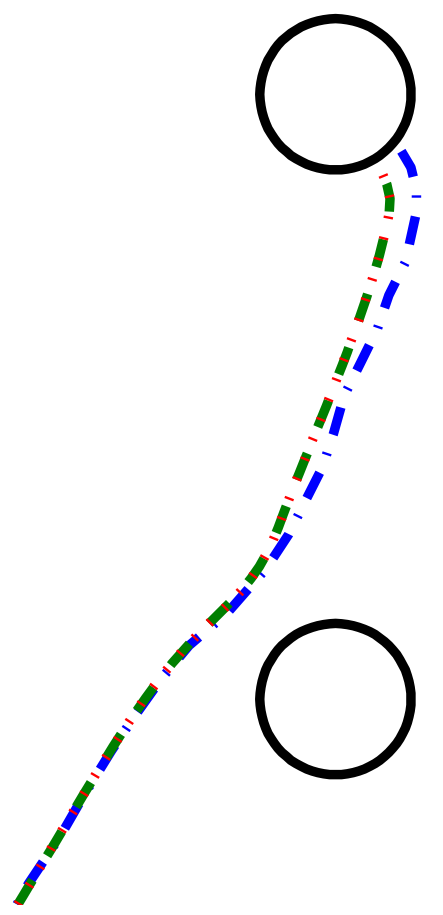

(c)

Figure 29: Crack evolution of the three cases 


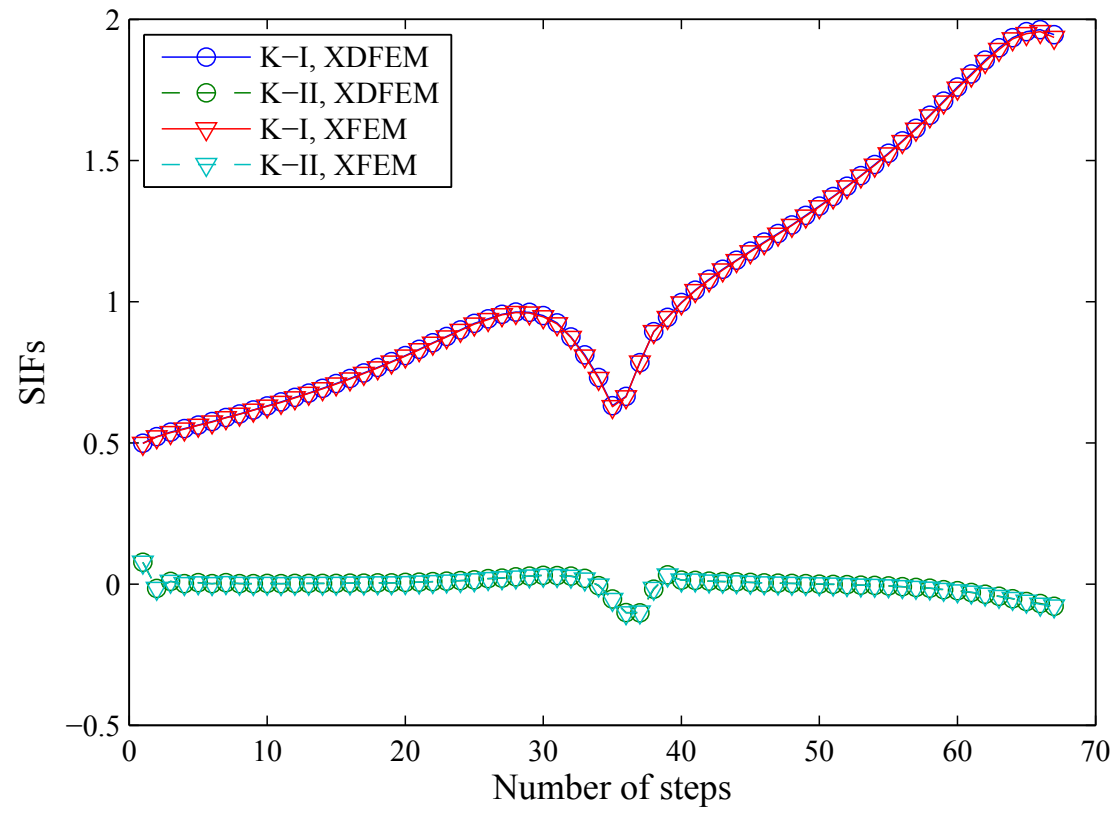

(a)

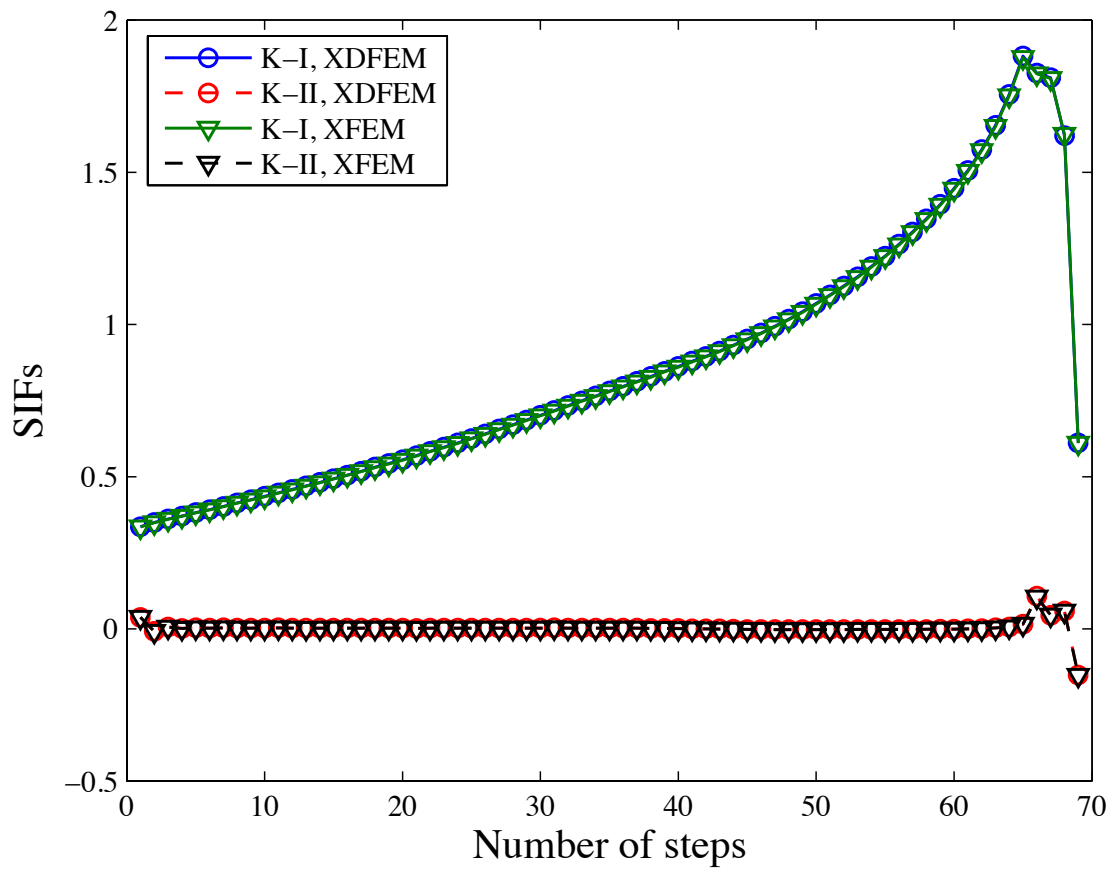

(b) 


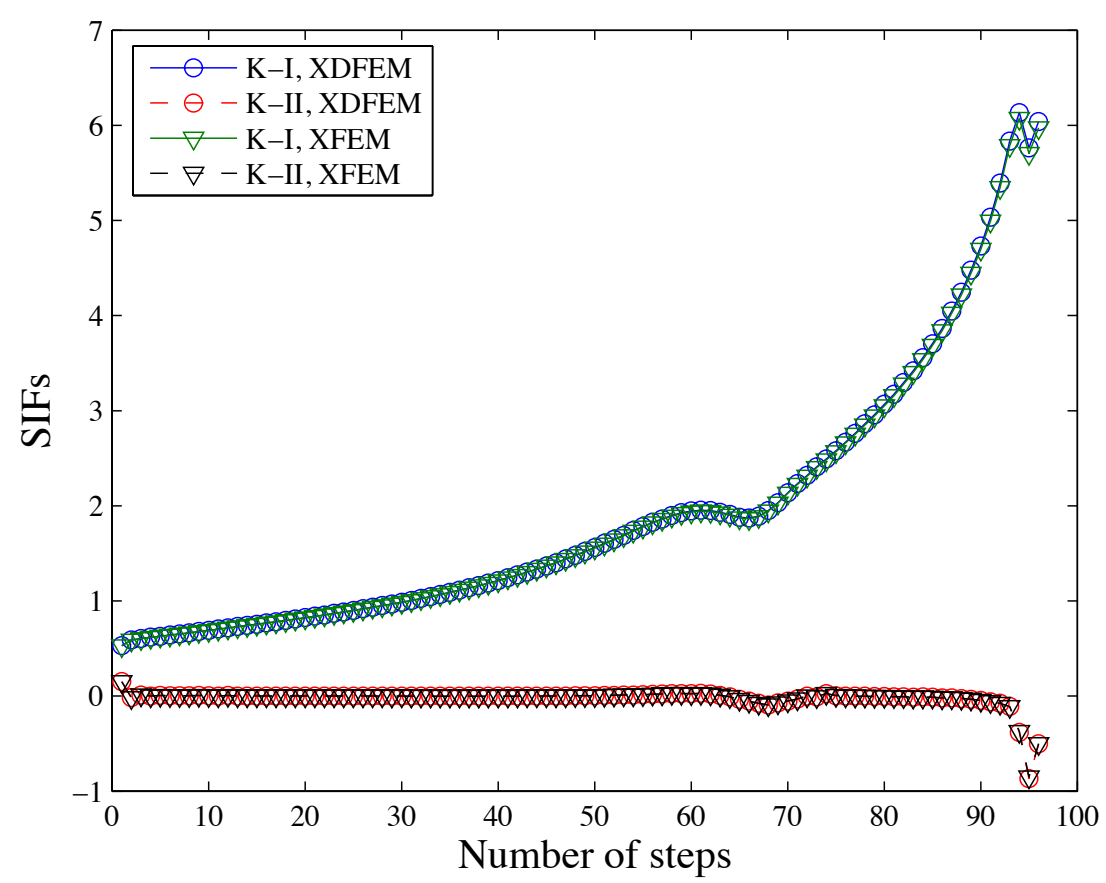

(c)

Figure 30: SIFs variation in three cases

\section{Conclusions}

This paper presented an enriched double-interpolation approximation method for linear elastic fracture and crack growth analysis. The double-interpolation approximation is constructed through two consequent stages of interpolation, i.e., the linear finite element interpolation for the first stage to produce an initial approximation field which will be utilized to reproduce the solution via a second interpolation with smooth nodal gradients. Several examples are tackled to explore the basic features of DFEM and XDFEM. The key points are summarized as follows:

- The precision of the solution field is almost improved by up to a level of $O\left(10^{-1}\right)$ error in both displacement and energy norm without increasing the total DOFs, due to the fact that the basis functions of the double-interpolation approximation have been enhanced through the embedment of area weighted 'average' gradients. Numerical tests showed that the double-interpolation method is more accurate than the Q4 finite element for the same model size, despite using a simplex mesh. Quadrilateral (hexahedral) mesh achieves higher accuracy while, simplex meshes are more convenient to generate in particular for moving boundaries requiring adaptivity. DFEM proves to unite the two factors together to provide an practical and efficient modeling technique.

- The convergence rate of the DFEM is shown to behave midway between linear finite elements and quadratic finite elements. The DFEM is more accurate than linear triangular Lagrange interpolants, less accurate than quadratic triangular elements, and offers a compromise between these two element classes. In contrast to common higher-order finite element, DFEM also provides $C^{1}$ continuity on most nodes. For continuum mechanics problems, it does not require any post-processing for recovering the nodal stresses. 


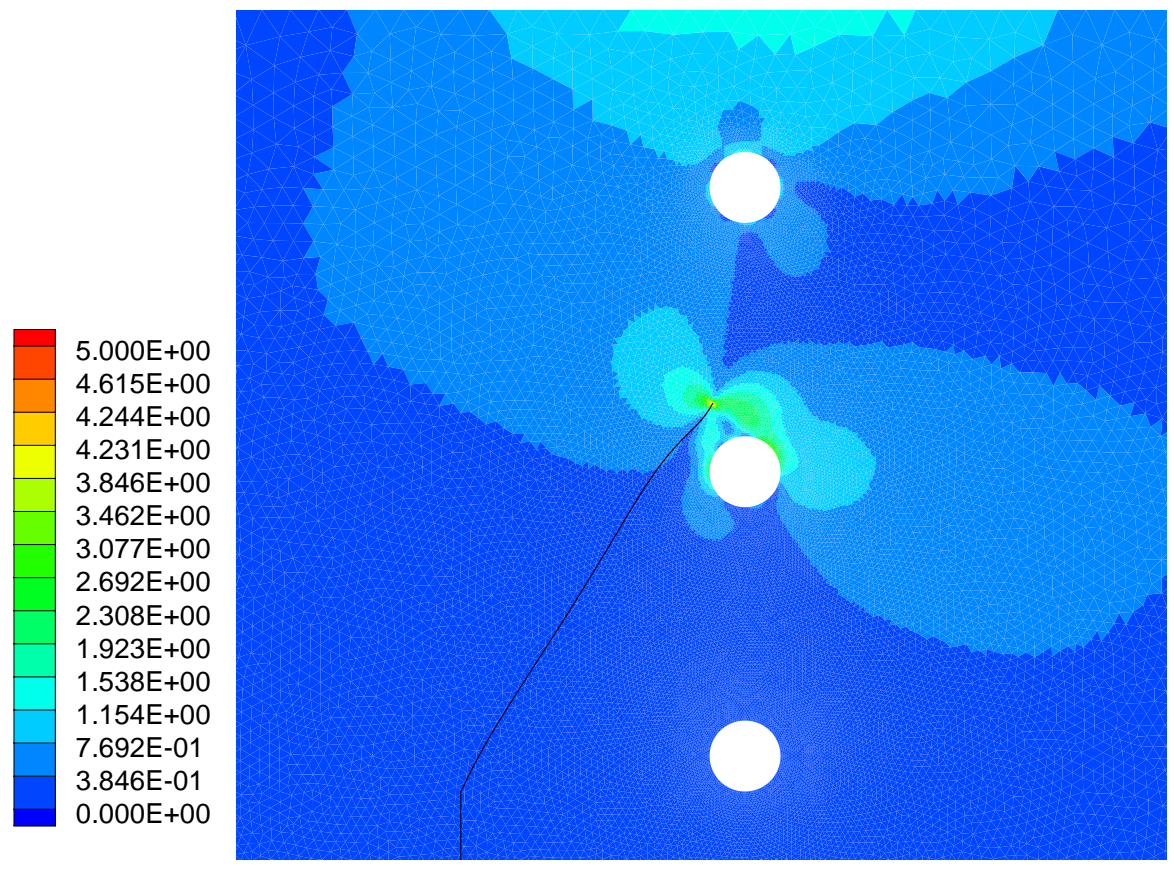

(a) XFEM, the 69th step

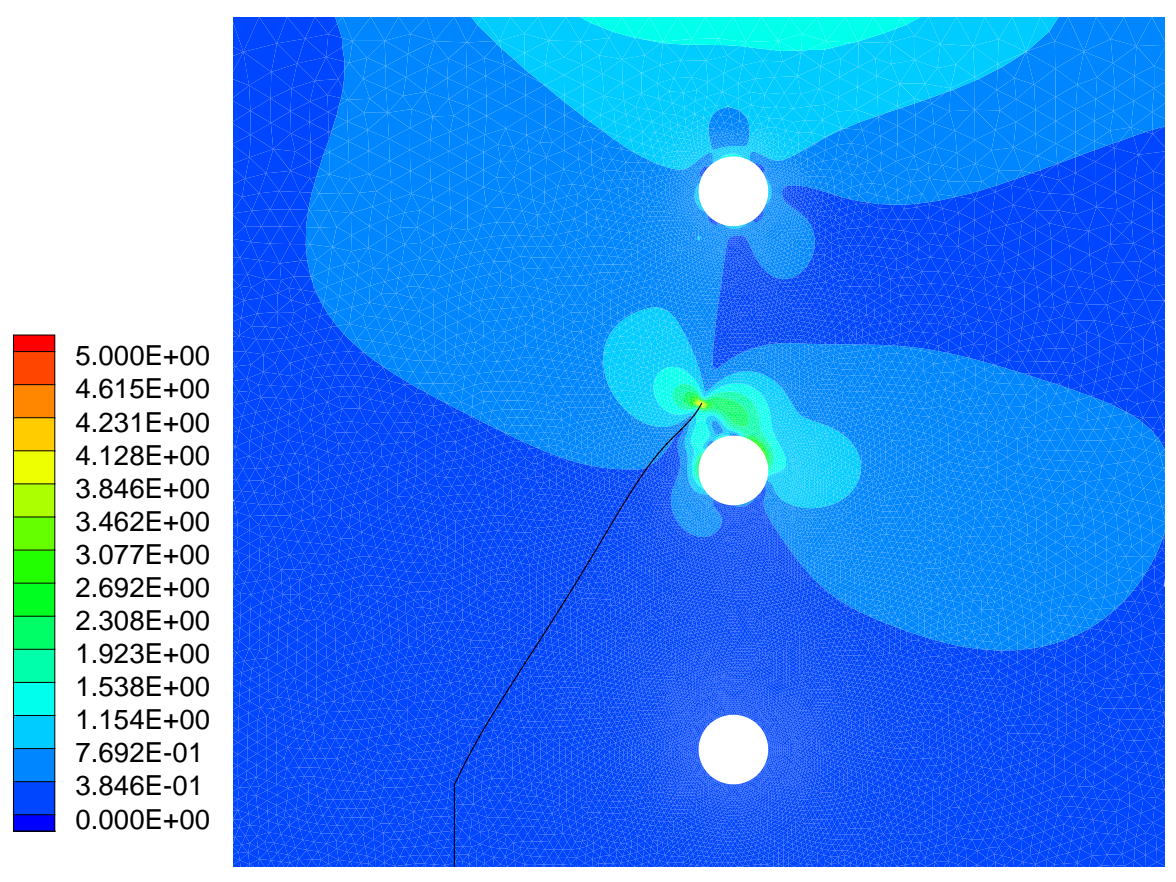

(b) XDFEM, the 69th step 


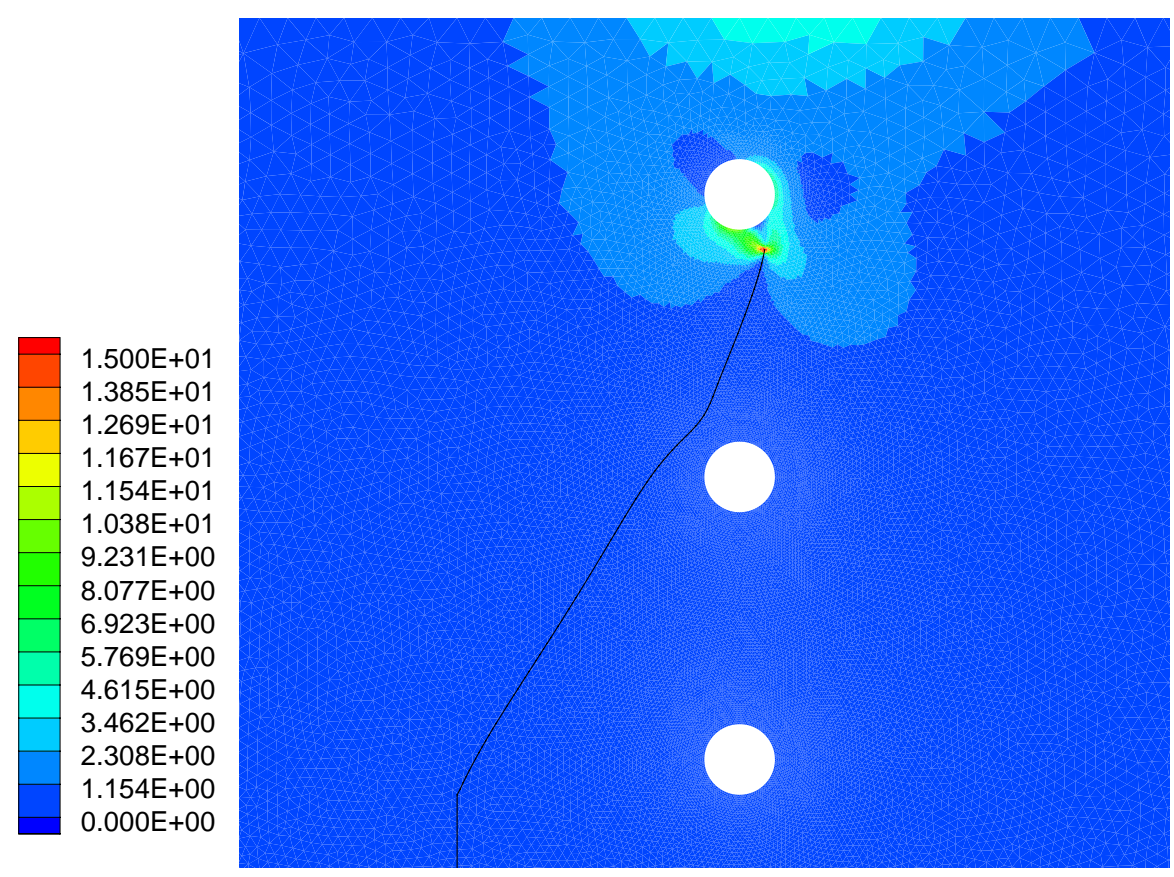

(c) XFEM, the 94th step

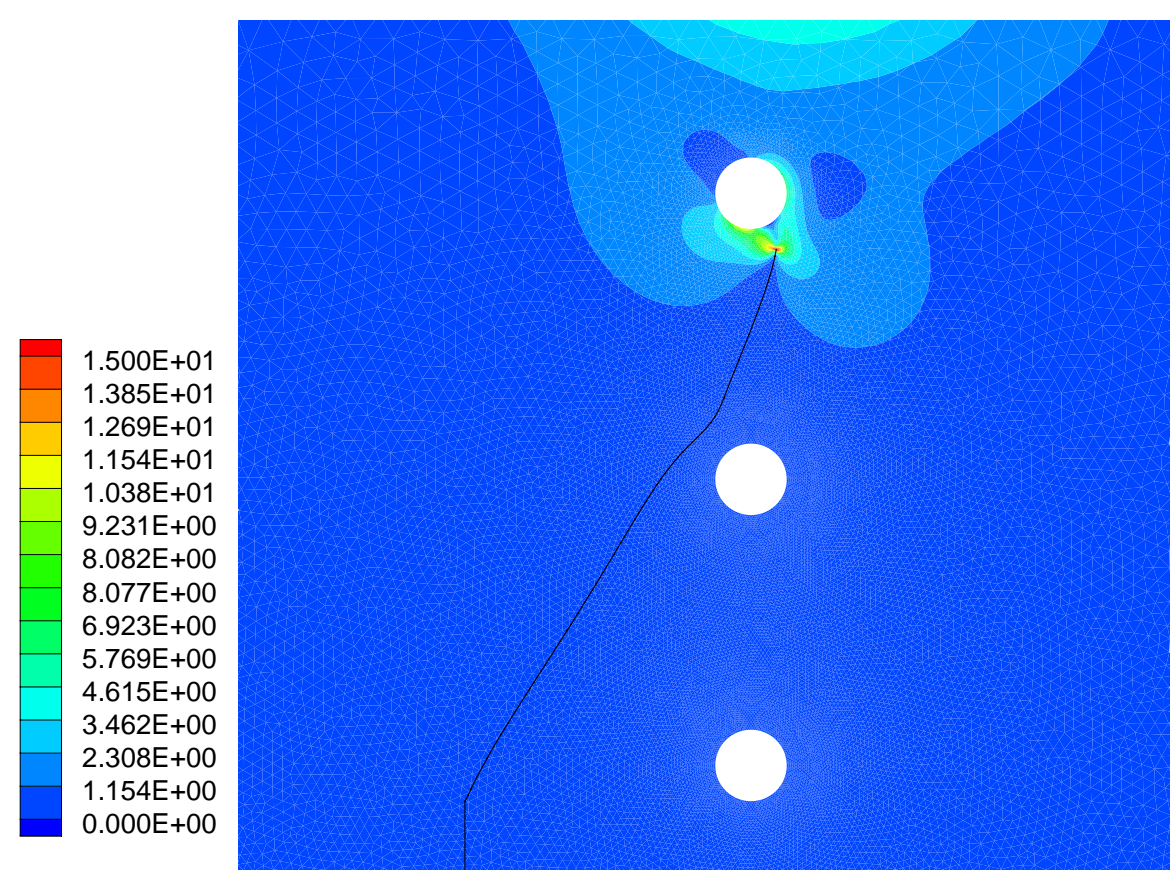

(d) XDFEM, the 94th step

Figure 31: Contour plots of Von Mises stress in case 3 
For fracture analysis, only the tip-enriched nodes require extra post-processing. Postprocessing procedure is thus unnecessary in DFEM and XDFEM, which improves the efficiency of the simulation and ensures all fields in the same space. This is expected to be useful for non-linear simulations.

- It should be highlighted that the major factor which hampers the efficiency of DFEM is the increased bandwidth issue which is caused by the introduction of the average gradients. When the element-by-element strategy is used, this extra time needed in searching the stiffness matrix because of the expanded bandwidth can however be saved.

- The XDFEM provides a robust solution to crack propagation problems analogous to XFEM, whilst providing a smoother stress field without post-processing. This could be useful in improving the accuracy in 3D fracture modeling, in which the precision of Lagrange-based XFEM is poor due to the low continuity of the solution.

The 3D XDFEM should be investigated to verify the accuracy of the solution with more elastic problems implementation. Zienkiewicz-Zhu error estimation [56] based on XDFEM is also an interesting topic for investigation. Further it would be beneficial to identify a procedure to maintain $C^{1}$ continuity at the tip-enriched nodes.

\section{Acknowledgements}

The authors would like to acknowledge the financial support of the Framework Programme 7 Initial Training Network Funding under grant number 289361 "Integrating Numerical Simulation and Geometric Design Technology" (FP7:ITN-INSIST).

\section{References}

[1] N Moës, J Dolbow, and T Belytschko. A finite element method for crack growth without remeshing. International Journal for Numerical Methods in Engineering, 46(1):131-150, 1999.

[2] N Moës, A Gravouil, and T Belytschko. Non-planar 3D crack growth by the extended finite element and level sets-Part I: Mechanical model. International Journal for Numerical Methods in Engineering, 53(11):2549-2568, 2002.

[3] A Gravouil, N Moës, and T Belytschko. Non-planar 3D crack growth by the extended finite element and level sets-Part II: Level set update. International Journal for Numerical Methods in Engineering, 53(11):2569-2586, 2002.

[4] N Sukumar, D L Chopp, E Béchet, and N Moës. Three-dimensional non-planar crack growth by a coupled extended finite element and fast marching method. International Journal for Numerical Methods in Engineering, 76(5):727-748, 2008.

[5] G Zi and T Belytschko. New crack-tip elements for XFEM and applications to cohesive cracks. International Journal for Numerical Methods in Engineering, 57(15):2221-2240, 2003.

[6] P M A Areias and T Belytschko. Non-linear analysis of shells with arbitrary evolving cracks using XFEM. International Journal for Numerical Methods in Engineering, 62(3):384-415, 2005. 
[7] T Belytschko, H Chen, J Xu, and G Zi. Dynamic crack propagation based on loss of hyperbolicity and a new discontinuous enrichment. International Journal for Numerical Methods in Engineering, 58(12):1873-1905, 2003.

[8] S Bordas and B Moran. Enriched finite elements and level sets for damage tolerance assessment of complex structures. Engineering Fracture Mechanics, 73(9):1176-1201, 2006.

[9] M Duflot and S P A Bordas. A posteriori error estimation for extended finite elements by an extended global recovery. International Journal for Numerical Methods in Engineering, 76(8):1123-1138, 2008.

[10] S P A Bordas and M Duflot. Derivative recovery and a posteriori error estimate for extended finite elements. Computer Methods in Applied Mechanics and Engineering, 196(3536):3381-3399, 2007.

[11] J J Ródenas, O A González-Estrada, J E Tarancón, and F J Fuenmayor. A recovery-type error estimator for the extended finite element method based on singular + smooth stress field splitting. International Journal for Numerical Methods in Engineering, 76(4):545-571, 2008 .

[12] J Panetier, P Ladevèze, and L Chamoin. Strict and effective bounds in goal-oriented error estimation applied to fracture mechanics problems solved with XFEM. International Journal for Numerical Methods in Engineering, 81(6):671-700, 2010.

[13] S Bordas, P V Nguyen, C Dunant, A Guidoum, and H Nguyen-Dang. An extended finite element library. International Journal for Numerical Methods in Engineering, 71(6):703$732,2007$.

[14] E Wyart, M Duflot, D Coulon, P Martiny, T Pardoen, J.-F. Remacle, and F Lani. Substructuring FE-XFE approaches applied to three-dimensional crack propagation. Journal of Computational and Applied Mathematics, 215(2):626-638, 2008.

[15] J Shi, D L Chopp, J Lua, N Sukumar, and T Belytschko. Abaqus implementation of extended finite element method using a level set representation for three-dimensional fatigue crack growth and life predictions. Engineering Fracture Mechanics, 77(14):2840-2863, 2010.

[16] D Paladim, N Sundarajan, and S Bordas. Stable extended finite element method: Convergence, Accuracy, Properties and Diffpack implementation. International conference on Extended Finite Element Methods, (Lyon, France), 2013.

[17] C A Duarte, O N Hamzeh, T J Liszka, and W W Tworzydlo. A generalized finite element method for the simulation of three-dimensional dynamic crack propagation. Computer Methods in Applied Mechanics and Engineering, 190(15-17):2227-2262, 2001.

[18] J Chessa, H Wang, and T Belytschko. On the construction of blending elements for local partition of unity enriched finite elements. International Journal for Numerical Methods in Engineering, 57(7):1015-1038, 2003.

[19] J E Tarancón, A Vercher, E Giner, and F J Fuenmayor. Enhanced blending elements for XFEM applied to linear elastic fracture mechanics. International Journal for Numerical Methods in Engineering, 77(1):126-148, 2009.

[20] R Gracie, H Wang, and T Belytschko. Blending in the extended finite element method by discontinuous Galerkin and assumed strain methods. International Journal for Numerical Methods in Engineering, 74(11):1645-1669, 2008. 
[21] T Fries. A corrected XFEM approximation without problems in blending elements. International Journal for Numerical Methods in Engineering, 75(5):503-532, 2008.

[22] G Ventura, R Gracie, and T Belytschko. Fast integration and weight function blending in the extended finite element method. International Journal for Numerical Methods in Engineering, 77(1):1-29, 2009.

[23] G Ventura. On the elimination of quadrature subcells for discontinuous functions in the eXtended Finite-Element Method. International Journal for Numerical Methods in Engineering, 66(5):761-795, 2006.

[24] S Natarajan, D R Mahapatra, and S P A Bordas. Integrating strong and weak discontinuities without integration subcells and example applications in an XFEM/GFEM framework. International Journal for Numerical Methods in Engineering, 83(3):269-294, 2010.

[25] L Chen, T Rabczuk, S P A Bordas, G R Liu, K Y Zeng, and P Kerfriden. Extended finite element method with edge-based strain smoothing (ESm-XFEM) for linear elastic crack growth. Computer Methods in Applied Mechanics and Engineering, 209-212:250-265, February 2012.

[26] P Laborde, J Pommier, Y Renard, and M Salaün. High-order extended finite element method for cracked domains. International Journal for Numerical Methods in Engineering, 64(3):354-381, 2005.

[27] E Béchet, H Minnebo, N Moës, and B Burgardt. Improved implementation and robustness study of the X-FEM for stress analysis around cracks. International Journal for Numerical Methods in Engineering, 64(8):1033-1056, 2005.

[28] A Menk and S P A Bordas. A robust preconditioning technique for the extended finite element method. International Journal for Numerical Methods in Engineering, 85(13):1609$1632,2011$.

[29] N A Fleck and J W Hutchinson. Strain Gradient Plasticity. Advances in Applied Mechanics, 33(C):295-361, 1997.

[30] P Krysl and T Belytschko. Analysis of thin shells by the Element-Free Galerkin method. International Journal of Solids and Structures, 33(20-22):3057-3080, 1996.

[31] G Engel, K Garikipati, T J R Hughes, M G Larson, L Mazzei, and R L Taylor. Continuous/discontinuous finite element approximations of fourth-order elliptic problems in structural and continuum mechanics with applications to thin beams and plates, and strain gradient elasticity. Computer Methods in Applied Mechanics and Engineering, 191(34):3669$3750,2002$.

[32] S A Papanicolopulos and A Zervos. A method for creating a class of triangular C 1 finite elements. International Journal for Numerical Methods in Engineering, 89(11):1437-1450, 2012.

[33] S A Papanicolopulos and A Zervos. Polynomial C1 shape functions on the triangle. Computers $\&$ Structures, 118(0):53-58, 2013.

[34] P Fischer, J Mergheim, and P Steinmann. On the C1 continuous discretization of nonlinear gradient elasticity: A comparison of NEM and FEM based on Bernstein-Bézier patches. International Journal for Numerical Methods in Engineering, 82(10):1282-1307, 2010. 
[35] T Belytschko, Y Y Lu, and L Gu. Element-free Galerkin methods. International Journal for Numerical Methods in Engineering, 37(2):229-256, 1994.

[36] W K Liu, S Jun, and Y F Zhang. Reproducing kernel particle methods. International Journal for Numerical Methods in Fluids, 20(8-9):1081-1106, 1995.

[37] O Davydov, A Sestini, and R Morandi. Local RBF Approximation for Scattered Data Fitting with Bivariate Splines. In DetlefH. Mache, József Szabados, and MarcelG. Bruin, editors, Trends and Applications in Constructive Approximation, volume 151 of ISNM International Series of Numerical Mathematics, pages 91-102. Birkhäuser Basel, 2005.

[38] K M Liew, X L Chen, and J N Reddy. Mesh-free radial basis function method for buckling analysis of non-uniformly loaded arbitrarily shaped shear deformable plates. Computer Methods in Applied Mechanics and Engineering, 193(3-5):205-224, January 2004.

[39] J G Wang and G R Liu. On the optimal shape parameters of radial basis functions used for 2-D meshless methods. Computer Methods in Applied Mechanics and Engineering, 191(23-24):2611-2630, 2002.

[40] G R Liu, G Y Zhang, Y T Gu, and Y Y Wang. A meshfree radial point interpolation method (RPIM) for three-dimensional solids. Computational Mechanics, 36(6):421-430, August 2005.

[41] M Arroyo and M Ortiz. Local maximum-entropy approximation schemes: a seamless bridge between finite elements and meshfree methods. International Journal for Numerical Methods in Engineering, 65(13):2167-2202, 2006.

[42] A Rosolen, D Millán, and M Arroyo. On the optimum support size in meshfree methods: A variational adaptivity approach with maximum-entropy approximants. International Journal for Numerical Methods in Engineering, 82(7):868-895, 2010.

[43] F Amiri, C Anitescu, M Arroyo, S P A Bordas, and T Rabczuk. XLME interpolants, a seamless bridge between XFEM and enriched meshless methods. Computational Mechanics, $53(1): 45-57,2014$.

[44] S P A Bordas, T Rabczuk, H Nguyen-Xuan, V P Nguyen, S Natarajan, T. Bog, D. M. Quan, and N. V. Hiep. Strain smoothing in FEM and XFEM. Computers 65 Structures, 88(23-24):1419-1443, December 2010.

[45] G R Liu, L Chen, T Nguyen-Thoi, K Y Zeng, and G Y Zhang. A novel singular nodebased smoothed finite element method (NS-FEM) for upper bound solutions of fracture problems. International Journal for Numerical Methods in Engineering, 83(11):1466-1497, 2010.

[46] N Vu-Bac, H Nguyen-Xuan, L Chen, S Bordas, P Kerfriden, R N Simpson, G R Liu, and T Rabczuk. A Node-Based Smoothed eXtended Finite Element Method (NS-XFEM) for Fracture Analysis. Computer Modeling in Engineering \& Sciences, 73(4):331-356, 2011.

[47] Y Jiang, T E Tay, L Chen, and X S Sun. An edge-based smoothed XFEM for fracture in composite materials. International Journal of Fracture, 179(1-2):179-199, 2013.

[48] S P A Bordas and S Natarajan. On the performance of strain smoothing for quadratic and enriched finite element approximations (XFEM/GFEM/PUFEM). International Journal for Numerical Methods in Engineering, 86(4-5):637-666, 2011. 
[49] C Zheng, S C Wu, X H Tang, and J H Zhang. A novel twice-interpolation finite element method for solid mechanics problems. Acta Mechanica Sinica, 26(2):265-278, June 2009.

[50] S C Wu, W H Zhang, X Peng, and B R Miao. A twice-interpolation finite element method (TFEM) for crack propagation problems. International Journal of Computational Methods, 09(04):1250055, 2012.

[51] S Timoshenko and J N Goodier. Theory of Elasticity. Engineering societies monographs. McGraw Hill Book Company, 1972.

[52] H M Westergaard. Bearing pressures and cracks. Journal of Applied Mechanics, 6:A49A53, 1939.

[53] A R Ingraffea and M Grigoriu. Probabilistic fracture mechanics: A validation of predictive capability. Department of Structure Engineering, Cornell University, Rep. 90-8, 1990.

[54] T N Bittencourt, P A Wawrzynek, A R Ingraffea, and J L Sousa. Quasi-automatic simulation of crack propagation for 2D LEFM problems. Engineering Fracture Mechanics, $55(2): 321-334,1996$.

[55] G Ventura, J X Xu, and T Belytschko. A vector level set method and new discontinuity approximations for crack growth by EFG. International Journal for Numerical Methods in Engineering, 54(6):923-944, 2002.

[56] O C Zienkiewicz and J Z Zhu. The superconvergent patch recovery and a posteriori error estimates. Part 1: The recovery technique. International Journal for Numerical Methods in Engineering, 33(7):1331-1364, 1992. 